\title{
The deacylase SIRT5 supports melanoma viability by regulating chromatin dynamics
}

William Giblin ${ }^{1,2}$, Lauren Bringman-Rodenbarger ${ }^{1 \#}$, Angela H. Guo ${ }^{1 \#}$, Surinder Kumar ${ }^{1 \#}$,

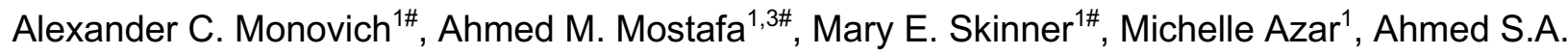
Mady $^{1}$, Carolina H. Chung ${ }^{4}$, Namrata Kadambi ${ }^{1}$, Keith-Allen Melong ${ }^{1}$, Ho-Joon Lee ${ }^{5}$, Li Zhang ${ }^{5}$, Peter Sajjakulnukit ${ }^{5}$, Sophie Trefely ${ }^{6,7}$, Erika L. Varner ${ }^{7}$, Sowmya lyer ${ }^{8}$, Min Wang ${ }^{1}$, James S. Wilmott $^{9}$, H. Peter Soyer ${ }^{10,11}$, Richard A. Sturm ${ }^{10}$, Antonia L. Pritchard ${ }^{12,13}$, Aleodor Andea ${ }^{1,14}$, Richard A. Scolyer ${ }^{9,15,16}$, Mitchell S. Stark ${ }^{10}$, David A. Scott ${ }^{17}$, Douglas R. Fullen ${ }^{1,14}$, Marcus W. Bosenberg $^{18}$, Sriram Chandrasekaran ${ }^{4,19,20,21}$, Zaneta Nikolovska-Coleska ${ }^{1,21}$, Monique E.

Verhaegen $^{14}$, Nathaniel W. Snyder ${ }^{7}$, Miguel N. Rivera ${ }^{8,22}$, Andrei L. Osterman ${ }^{17}$, Costas A. Lyssiotis $^{5,21,23}$, and David B. Lombard ${ }^{1,21,24^{*}}$

${ }^{1}$ Department of Pathology, University of Michigan, Ann Arbor, MI 48109 USA

${ }^{2}$ Department of Human Genetics, University of Michigan, Ann Arbor, MI 48109 USA

${ }^{3}$ Department of Biochemistry, Faculty of Pharmacy, Ain Shams University, Cairo, Egypt

${ }^{4}$ Department of Biomedical Engineering, University of Michigan, Ann Arbor, MI 48109 USA

${ }^{5}$ Department of Molecular and Integrative Physiology, University of Michigan, Ann Arbor, MI 48109 USA

${ }^{6}$ Department of Cancer Biology, University of Pennsylvania, Perelman School of Medicine, Philadelphia, PA 19104 USA

${ }^{7}$ Center for Metabolic Disease Research, Department of Microbiology and Immunology, Temple University, Lewis Katz School of Medicine, Philadelphia, PA 19140 USA

${ }^{8}$ Department of Pathology and MGH Cancer Center, Massachusetts General Hospital and Harvard Medical School, Boston, MA 02115 USA

${ }^{9}$ Melanoma Institute Australia, The University of Sydney, Sydney, New South Wales, 2065 Australia 
${ }^{10}$ The University of Queensland Diamantina Institute, The University of Queensland,

Dermatology Research Centre, Brisbane, QLD 4102 Australia

${ }^{11}$ Department of Dermatology, Princess Alexandra Hospital, Brisbane, Queensland 4102

Australia

${ }^{12}$ Institute of Health Research and Innovation, University of the Highlands and Islands, An

Lóchran, 10 Inverness Campus, Inverness, IV2 5NA United Kingdom

${ }^{13}$ Oncogenomics, QIMR Berghofer Medical Research Institute, Brisbane, Queensland, 4006

Australia

${ }^{14}$ Department of Dermatology, University of Michigan, Ann Arbor, MI 48109 USA

${ }^{15}$ Tissue Pathology and Diagnostic Oncology, Royal Prince Alfred Hospital, and NSW

Pathology, Sydney, New South Wales, 2050 Australia

${ }^{16}$ Faulty of Medicine and Health, The University of Sydney, Sydney, New South Wales, 2006,

Australia

${ }^{17}$ Sanford Burnham Prebys Medical Discovery Institute, La Jolla, California 92037 USA

${ }^{18}$ Departments of Pathology and Dermatology, Yale University School of Medicine, New Haven, CT 06510 USA

${ }^{19}$ Program in Chemical Biology, University of Michigan, Ann Arbor, MI 48109 USA

${ }^{20}$ Center for Computational Medicine and Bioinformatics, University of Michigan, Ann Arbor, MI 48109 USA

${ }^{21}$ Rogel Cancer Center, University of Michigan Medical School, Ann Arbor, MI 48109 USA

${ }^{22}$ Broad Institute of Harvard and MIT, Cambridge, MA 02142 USA

${ }^{23}$ Division of Gastroenterology, Department of Internal Medicine, University of Michigan, Ann

Arbor, MI 48109 USA

${ }^{24}$ Institute of Gerontology, University of Michigan, Ann Arbor, MI 48109 USA

\#These authors contributed equally. 
${ }^{*}$ Correspondence:

University of Michigan

3015 BSRB

109 Zina Pitcher Place

Ann Arbor, Ml 48109

(734) 615-0498

davidlom@med.umich.edu

Conflict of interest:

MWB is a consultant for Eli Lilly and Company. RAS reports receiving fees for professional services from QBiotics, Merck Sharp \& Dohme, GlaxoSmithKline, Bristol-Myers Squibb, Dermpedia, Novartis, Myriad, NeraCare and Amgen. CAL is an inventor on patents pertaining to KRAS regulated metabolic pathways, redox control pathways in pancreatic cancer, and targeting GOT1 as a therapeutic approach. CAL is also an author on a provisional patent application concerning the development of technologies that integrate the metabolic flux assay with cellular high content image analysis. DBL reports ownership of the equivalent in voting stock or share of ABBV and GILD. 


\begin{abstract}
Cutaneous melanoma remains the most lethal skin cancer, and ranks third among all malignancies in terms of years of life lost. Despite the advent of immune checkpoint and targeted therapies, only roughly half of patients with advanced melanoma achieves a durable remission. SIRT5 is a member of the sirtuin family of protein deacylases that regulate metabolism and other biological processes. Germline Sirt5 deficiency is associated with mild phenotypes in mice. Here we show that SIRT5 is required for proliferation and survival across all cutaneous melanoma genotypes tested, as well as uveal melanoma, a genetically distinct melanoma subtype that arises in the eye and is incurable once metastatic. Likewise, SIRT5 is required for efficient tumor formation by melanoma xenografts and in an autochthonous mouse Braf;Pten-driven melanoma model. Via metabolite and transcriptomic analyses, we find that SIRT5 is required to maintain histone acetylation and methylation levels in melanoma cells, thereby promoting proper gene expression. SIRT5-dependent genes notably include MITF, a key lineage-specific survival oncogene in melanoma, and the c-MYC proto-oncogene. SIRT5 may represent a novel, druggable genotype-independent addiction in melanoma.
\end{abstract}




\section{Introduction}

Cutaneous melanoma remains the most lethal skin cancer. In 2021, there will be an estimated 106,110 new melanoma cases and 7,180 melanoma-related deaths in the US (1) Melanoma incidence is rising (2), and melanoma ranks third among all cancers in terms of years of life lost $(3,4)$. Despite the advent of immune checkpoint and targeted therapies, only about half of patients with advanced melanoma achieves long-term remission, even with optimal immune checkpoint therapy (5). Uveal melanoma represents a genetically and clinically distinct subtype of melanoma that arises in the eye, and currently has no effective treatment options once metastatic (6). New therapeutic strategies for advanced melanoma are urgently needed.

Mammalian sirtuins are a family of seven $\mathrm{NAD}^{+}$-dependent lysine deacylases that regulate diverse processes to promote cellular and organismal homeostasis and stress responses. Among these proteins, Sirtuin 5 (SIRT5) has remained a somewhat enigmatic and poorly characterized sirtuin. SIRT5 is atypical, in that it lacks robust deacetylase activity, and primarily functions to remove succinyl, malonyl, and glutaryl modifications from lysines on its target proteins, in mitochondria and throughout the cell, thereby regulating multiple metabolic pathways (7-14).

SIRT5-deficient mice are viable, fertile, and mostly healthy $(15,16)$, with the most prominent effects described to date occurring in the myocardium (17). Sirt5 knockout (KO) mice are more susceptible to ischemia-reperfusion injury and exhibit impaired recovery of cardiac function compared to wild type (WT) mice (18). Aged Sirt5 KO mice develop cardiac hypertrophy and mildly impaired ejection fraction (19). Whole-body Sirt5 KOs, but not cardiomyocyte-specific KOs, show increased lethality in response to cardiac pressure overload $(20,21)$. Overall, however, the lack of strong phenotypes associated with SIRT5 loss-of-function in normal tissues 
has hindered progress in understanding the biological significance of SIRT5 and its target posttranslational modifications.

Multiple sirtuins are now linked to neoplasia, as tumor suppressors and/or oncogenes, in a context-specific manner (22). In the context of melanoma, genetic inhibition of SIRT1 in human melanoma cell lines induces senescence and sensitizes drug-resistant cells to vemurafenib, an FDA-approved therapy for the treatment of BRAF-mutant melanoma (23). Conversely, genetic SIRT2 inhibition results in vemurafenib resistance in BRAF-mutant melanoma cells by altering MEK/ERK signaling (24). SIRT3 has likewise been reported to play an oncogenic role in melanoma. Reduction of SIRT3 levels in human melanoma lines results in decreased viability, increased senescence and impaired xenograft formation (25). SIRT6 is upregulated in melanoma cells and tissue samples, and SIRT6 depletion in melanoma cell lines results in reduced colony formation and proliferation (26). Paradoxically, SIRT6 haploinsufficiency induces resistance to targeted therapies in BRAF-mutant melanoma cells by regulating IGF/AKT signaling (27).

The functions of SIRT5 in cancer are not well understood, and a subject of active investigation (7). For example, SIRT5 promotes chemoresistance in non-small cell lung carcinoma cells by enhancing NRF2 activity and expression of its targets involved in cellular antioxidant defense (28). SIRT5 promotes Warburg-type metabolism in lung cancer cells by negatively regulating SUN2, a member of the linker of nucleoskeleton and cytoskeleton complex (29). SIRT5 suppresses levels of reactive oxygen species (ROS) via desuccinylation of multiple targets (superoxide dismutase 1, glucose-6-phosphate dehydrogenase, and isocitrate dehydrogenase (IDH) 2), thereby promoting growth of lung cancer cell lines in vitro $(30,31)$. SIRT5 also plays an important role in facilitating tumor cell growth by desuccinylating serine 
hydroxymethyltransferase 2 (SHMT2), which catalyzes the reversible, rate-limiting step in serine catabolism, providing methyl groups for cellular methylation reactions via one-carbon metabolism (1CM) (32). Another study indicated that SIRT5 promotes hepatocellular carcinoma (HCC) proliferation and invasion by targeting the transcription factor E2F1 (33). Similarly, it was recently reported that SIRT5 suppresses apoptosis by deacetylation of cytochrome C, thereby promoting HCC growth (34). SIRT5 also promotes breast cancer tumorigenesis by desuccinylating and stabilizing glutaminase (35), an enzyme that catalyzes the conversion of glutamine to glutamate, which supports the metabolic demands of tumorigenesis (36). Another recent publication showed that SIRT5 promotes breast cancer growth in part by suppressing ROS, and described selective SIRT5 inhibitors that markedly impaired tumor growth in vivo (37). In contrast, SIRT5 opposes malignant phenotypes associated with expression of mutant IDH, which generates the novel oncometabolite R-2-hydroxyglutarate, thereby perturbing the epigenome (38). IDH mutant glioma cells show increased protein succinylation, exhibit mitochondrial dysfunction and are resistant to apoptosis. Ectopically expressed SIRT5 in these cells impaired their growth in vitro and in vivo. Another recent report indicates that SIRT5 inactivates STAT3, thus suppressing mitochondrial pyruvate metabolism in lung cancer (39).

Here, we identify a critical requirement for SIRT5 in melanoma cell survival, through chromatin regulation. In all cutaneous and uveal melanoma cell lines tested, from both humans and mice and with varied genetic drivers, SIRT5 depletion resulted in rapid loss of proliferative capacity and cell death. Likewise, SIRT5 loss reduced melanoma formation in xenograft and autochthonous mouse melanoma models. Via transcriptomic analysis, we identified a core set of genes that responds to SIRT5 depletion. Among these, MITF, an essential lineage-specific transcription factor in melanoma, is downregulated, along with expression of its targets (40). SIRT5 loss is also associated with reduced expression of $c-M Y C$, a well-described protooncogene that is often overexpressed in metastatic melanoma and melanoma cell lines, which 
plays an important role in therapeutic resistance $(41,42)$. We link the effects of SIRT5 depletion on gene expression to alterations in histone acetylation and methylation induced by metabolic changes occurring in the context of SIRT5 loss-of-function. Taken together, our results identify SIRT5 as a novel genotype-independent dependency in melanoma cells, likely exerting its effects via chromatin modifications and gene regulation. Given the modest effects of SIRT5 loss-of-function in normal tissues, SIRT5 may represent an attractive therapeutic target in melanoma and potentially other cancer types. 


\section{Results}

\section{The chromosomal region encompassing SIRT5 shows frequent copy number gain in}

\section{human melanoma}

In humans, the SIRT5 gene localizes to chromosome $6 \mathrm{p} 23$. The $6 \mathrm{p}$ region exhibits frequent copy number gain in melanoma, an event associated with an adverse prognosis, both in melanoma (43) and other cancers (44). To confirm that gain of the SIRT5 locus specifically occurs in human melanomas, we mined TCGA (The Cancer Genome Atlas) data (45) using cBioportal, and observed that copy number gain or amplification of SIRT5 was present in 55\% of melanoma cases, whereas SIRT5 deletion or mutation was rare (Figure 1A, S1A, and S1B). Increased SIRT5 copy number also correlated with increased SIRT5 mRNA expression in these samples (Figure S1C). In contrast, the presence of extra copies of the other six sirtuins was much less common in melanoma (Figure $1 \mathrm{~A}$ and $\mathrm{S} 1 \mathrm{~A}$ ). Activating mutations in BRAF and NRAS represent the most common oncogenic drivers in cutaneous melanoma (46). SIRT5 gain or amplification was observed in melanomas with either driver, and in melanomas with the less common driver mutation, NF1 (Figure 1A). Increased SIRT5 copy number was associated with moderately worsened overall survival ( $p=0.0097$; Figure 1B), although not progression-free survival (Figure S1D).

To assess further the status of the SIRT5 locus in melanoma, we performed fluorescence in situ hybridization (FISH) analysis of SIRT5 and the centromere of chromosome 6 in an independent group of melanoma samples (Figure 1C and S1E). Consistent with TCGA data, increased SIRT5 copy number was observed in 38\% (12/32) of cases analyzed overall, with coamplification of SIRT5 and the centromere of chromosome 6 present in 16\% (5/32) of cases. Similarly, using comparative genomic hybridization analysis in yet another independent group of melanoma samples, gain of the SIRT5 locus was present in $27 \%$ of melanoma cases analyzed (37/139), the most frequent gain among any of the sirtuins (Figure 1D). We also note that the 
SIRT7 locus was amplified in a substantial fraction of melanoma cases. SIRT7 promotes DNA repair by deacetylating and desuccinylating histones (47), however it is not currently known what role SIRT7-mediated deacylation might play in melanomagenesis.

We then interrogated SIRT5 mRNA expression in melanomas of varied depth of invasion, and found that increased SIRT5 mRNA expression occurred in melanomas of greater Clark's level, which are more clinically aggressive and confer a worse prognosis $(48,49)$ (Figure 1E). Similarly, we examined SIRT5 protein expression in tissue microarrays containing examples of benign and dysplastic nevi, as well as localized and metastatic melanomas. We found by immunohistochemistry that SIRT5 protein was overexpressed in melanomas relative to benign melanocytic lesions (Figure 1F).

To characterize the stage of melanogenesis at which SIRT5 gain occurs, we screened a panel of genomically characterized benign and dysplastic nevi $(n=30)(50)$ for SIRT5 somatic mutations and copy number aberrations. No deleterious point mutations were identified in SIRT5; however, there was evidence of regional loss of heterozygosity encompassing the SIRT5 locus in 3/30 benign nevi (10\%) assayed (Table S1). However, no SIRT5 copy number gain or amplification was identified in any of the nevus samples, supporting the idea that SIRT5 amplification represents a relatively late event in melanomagenesis. This is consistent with the known rarity of such genomic events in nevi $(50,51)$. Overall, these data show that gain or amplification of SIRT5 is a common genomic event in melanoma but not nevi.

\section{SIRT5 is required for survival of BRAF ${ }^{\mathrm{V} 600 \mathrm{E}}$ and NRAS ${ }^{\mathrm{Q61R}}$ melanoma cells}

We assessed the potential requirement of SIRT5 in melanoma cells using a panel of $10 B R A F$ or NRAS mutant melanoma cell lines (Table S2). SIRT5 protein was readily detectable by immunoblot in all cell lines tested (Figure S2A). We initially depleted SIRT5 using two lentiviral 
shRNAs targeting distinct regions of the SIRT5 mRNA (knockdown (KD) 1 and KD2) (11). Although predominantly mitochondrial, SIRT5 is also present in the cytosol and the nucleus (11), and was efficiently depleted from all of these compartments upon SIRT5 shRNA transduction in all cell lines tested (Figure S2B and S2C). In both BRAF ${ }^{\mathrm{V} 600 \mathrm{E}}$ and NRAS ${ }^{\mathrm{Q} 61 \mathrm{R}}$ cells, SIRT5 depletion induced rapid loss of proliferation over the course of 7 days (Figure $2 \mathrm{~A}$ and S2D). Similar results were obtained in an in vitro colony forming assay (Figure 2B). Vemurafenib is a targeted therapy FDA-approved for treatment of BRAF-mutant melanoma. Patients treated with targeted therapies often rapidly relapse with drug-resistant disease (52). SIRT5 inhibition in a vemurafenib-resistant derivative of the melanoma cell line SK-MEL-239, SK-MEL-239VR, induced rapid loss of proliferation upon SIRT5 KD, indicating that these vemurafenib-resistant cells retained SIRT5 dependency (Figure 2A and S2E). To complement shRNA-based studies, and to further evaluate the requirement of melanoma cells for SIRT5, we mutated the SIRT5 locus via CRISPR-Cas9, using four distinct guide RNAs (gRNAs, G1-G4) targeting SIRT5. Consistent with results obtained using shRNA, a dramatic reduction in colony formation was observed in SIRT5 mutant populations compared to control (Figure 2C). In contrast, SIRT5 KD in several ovarian cancer cell lines did not induce loss of viability as seen in melanoma, indicating that SIRT5 depletion is tolerated in some cancer types (Figure S2F and S2G).

\section{Loss of SIRT5 leads to apoptotic cell death in cutaneous and uveal melanoma cells}

We evaluated the mechanism of cellular attrition induced by SIRT5 loss-of-function. SIRT5 depletion in melanoma cells induced cleavage of caspase 3 (Figure $3 \mathrm{~A}$ and 3B) and induction of Annexin V positivity (Figure 3C and 3D). Importantly, SIRT5 depletion also blocked proliferation and induced cleavage of caspase 3 in uveal melanoma cell lines (Figure 3B; top panel; representative of 4/4 uveal melanoma cell lines tested; see Table S2). Cell loss and induction of caspase 3 cleavage at 96 hrs. post-transduction were also observed, to varying degrees, with 
an additional 3 unique shRNAs targeting human SIRT5 (Figure 3B; middle panel), and 5 unique shRNAs targeting murine SIRT5 in YUMM5.2, a mouse melanoma cell line (53) (Figure 3B; bottom panel).

Non-apoptotic mechanisms of cell death have been described in melanomas and other cancer types, specifically: autophagic, ER-stress induced, necroptosis and pyroptosis (54). We evaluated whether SIRT5 KD in melanoma cell lines harboring either BRAF or NRAS mutations induced these alternate cell death pathways. We did not observe increased conversion of LC3 A/B I to LC3 A/B II or SQSTM1/p62 loss (Figure S3A), increased expression of PERK, Calnexin, IRE1 alpha or PDI (Figure S3B), phosphorylation of either MLKL or RIP (Figure S3C), or accumulation of gasdermin D or caspase 1 cleavage products (Figure S3D), although there was some variation observed between different SIRT5 KD constructs. Thus, SIRT5 is required for survival and proliferation of multiple genetically diverse melanoma cell lines in vitro, in both human and mouse, and for survival of human uveal melanoma cells.

\section{SIRT5 supports robust melanoma tumor formation in vivo}

To investigate the potential requirement for SIRT5 to support melanoma tumor development in vivo, we initially employed a xenograft assay. Immediately following transduction with SIRT5 shRNAs, A2058 melanoma cells were subcutaneously injected into the flanks of female NOD/SCID mice (Figure S4A); tumor growth was followed by serial measurement of tumor volume (Figure 4A; left panel). SIRT5 depletion greatly impaired tumor growth and reduced tumor size at endpoint relative to controls (Figure 4A; right panel, 4B and S4B).

To examine the role of SIRT5 in melanoma development in a more physiologic, immunocompetent context, we crossed Sirt5 KO mice to a commonly-used mouse melanoma model, the Braf ${ }^{C A} ;$ Pten $^{f / f t} ;$ Tyr::CreER strain (55). Topical application of 4-hydroxytamoxifen 
$(4 \mathrm{HT})$ in this system induces activated BRAF expression and ablation of Pten in melanocytes, resulting in melanoma development. In males, SIRT5-deficient mice showed an approximately 3-fold reduction in tumor mass on average (WT: $1.005 \pm 0.618 \mathrm{~g}$ vs. KO: $0.323 \pm 0.198 \mathrm{~g}, \mathrm{p}<0.05$, (Figure 4C and 4D). In our colony, female mice showed rapid ulceration of even small melanoma tumors following induction (not shown), requiring euthanasia of the host and rendering it difficult to assess the effects of SIRT5 in melanoma in females. Thus, SIRT5 promotes human and mouse melanoma growth, both in cell culture and in vivo.

\section{Neither glucose nor glutamine metabolism are greatly altered by SIRT5 loss}

Initially, we considered the possibility that SIRT5 depletion might induce global metabolic collapse and energetic catastrophe in melanoma. SIRT5 has been reported to promote mitochondrial respiration $(56,57)$ and glycolysis $(14)$. We previously showed that SIRT5 suppresses mitochondrial respiration through Pyruvate Dehydrogenase and Complex II in 293T cells and liver mitochondria (11), a finding recapitulated in some systems (39), but not others $(56,57)$. We used the XFe96 Extracellular Flux Analyzer to assess the effects of SIRT5 depletion on cellular bioenergetics in melanoma cells. Relative to SIRT5-proficient controls, SIRT5 KD A2058 or A375 cells did not show consistent changes in the extracellular acidification rate (ECAR), a measure of cellular glycolysis (Figure 5A). Likewise, glucose-dependent mitochondrial oxygen consumption rate (OCR), ATP production, and mitochondrial membrane potential were not consistently affected by SIRT5 depletion (Figure 5B-5D).

Melanoma and many other cancer types replenish the TCA cycle in part via glutaminolysis (5861). In this pathway, glutaminase (GLS) catalyzes conversion of glutamine to glutamate, generating carbon and nitrogen to fuel the metabolic demands of tumorigenesis. In breast cancer cells, SIRT5 desuccinylates GLS to stabilize it, protecting it from ubiquitination and subsequent degradation. Loss of SIRT5 resulted in decreased GLS expression, exogenous 
glutamine consumption, glutamine-derived intracellular metabolite levels, and cellular proliferation (35). These findings, along with reports that inhibiting glycolysis or glutamine metabolism sensitizes melanoma cells to cell death, prompted us to investigate a potential role for SIRT5 in promoting glutamine metabolism in melanoma $(62,63)$. We cultured control or SIRT5 KD A2058 cells in medium containing glutamine labeled with stable isotopes $\left(\left[{ }^{15} \mathrm{~N}_{2}\right]-\right.$ glutamine or $\left[{ }^{13} \mathrm{C}_{5}\right]$-glutamine) or in medium containing $\left[{ }^{13} \mathrm{C}_{6}\right]$-glucose, and measured both labeling derived from ${ }^{13} \mathrm{C}$ or ${ }^{15} \mathrm{~N}$ and total quantities of cellular metabolites. Following SIRT5 KD, the fractional labeling of glutamine derived metabolites (glutamate, aspartate and TCA cycle metabolites) modestly decreased when cells were cultured in $\left[{ }^{13} \mathrm{C}_{5}\right]$-glutamine, but showed corresponding (or compensatory) increases in labeling when cultured in ${ }^{13} \mathrm{C}_{6}$-glucose (Figure S5A-S5B). Labeling derived from ${ }^{15} \mathrm{~N}_{2}$-glutamine was inconsistent between the two KD constructs analyzed in A2058 cells (Figure S5C). Overall, these results are consistent with previous results showing that SIRT5 promotes glutaminase activity (35). However, importantly, total cellular amounts of glutamate, aspartate, TCA cycle or other metabolites were not consistently reduced by SIRT5 loss (Figure S5D), indicating that although the depletion of SIRT5 may reduce glutaminase activity, this effect is insufficient to compromise levels of essential cellular metabolites. In parallel studies, glutamine-dependent mitochondrial OCR, and GLS protein levels, were assessed, and were not appreciably altered by SIRT5 depletion across multiple melanoma cell lines (Figure S5E and S5F). Moreover, incubation of SIRT5 KD A2058 cells with exogenous non-essential amino acids plus alpha-ketoglutarate - interventions that can rescue defects in glutamine catabolism (61) - in the context of SIRT5 KD failed to rescue the proliferative defect observed upon SIRT5 loss (Figure S5G). Taken together, these data indicate that neither glycolysis nor glutamine metabolism represent major SIRT5 target pathways in promoting melanoma viability. 


\section{Transcriptomic analysis reveals a requirement for SIRT5 in supporting MITF and MITF target gene expression}

To understand the requirement of melanoma cells for SIRT5 mechanistically, RNA-seq based transcriptomic analysis was performed on three cutaneous melanoma cell lines (A2058, A375 and SK-MEL-2), each subjected to SIRT5 depletion using two distinct shRNAs (Table S3). A gene was scored as differentially expressed only if it was consistently altered in all biological replicates by both independent SIRT5 shRNAs. We identified core sets of protein-coding genes whose expression responded to SIRT5 KD, many of which overlapped among the cell lines (Figure 6A and 6B). We then asked if any significant differentially expressed genes (DEGs) found in our SIRT5 RNA-seq dataset correlated with SIRT5 expression in TCGA data of clinical human skin cutaneous melanoma samples (see Methods for details). The most significant positively correlated overlapping DEG in this analysis was the Melanocyte Inducing Transcription Factor (MITF) (Figure 6C).

MITF, a key lineage-specific oncogenic transcription factor in melanoma that plays crucial roles in development and proliferation of melanocytes (40). MITF is expressed in human melanomas, and MITF amplification, present in a subset of melanoma tumors, portends a poor prognosis (64). Melanomas exhibit a wide range of MITF expression levels (65-67). In cutaneous melanoma cells with robust baseline MITF expression, MITF protein and mRNA expression declined markedly in response to SIRT5 KD (Figure 6D and 6E), associated with decreased expression of MITF's canonical targets: genes involved in metabolism (PPARGC1A), melanocytic differentiation (TYR, MLANA), cell survival (BCL2) and others (Figure 6E). A trend towards a reduced MITF gene expression profile was also observed in A375 cells, which have low baseline MITF expression, upon SIRT5 KD (Figure S6A). Decreased SIRT5, MITF and MITF target gene expression was validated by qRT-PCR in A2058 cells, and to a lesser degree, 
in SK-MEL-2 (Figure S6B). We also observed a decrease in MITF protein levels upon SIRT5 KD in MP-41 cells, a uveal melanoma line (Figure 6D).

To assess the potential relationship between SIRT5 and MITF in a more physiologic, non-lossof-function setting, we mined TCGA data, to test whether any correlation exists between SIRT5 and MITF mRNA expression in melanoma clinical samples. Consistent with the RNA-seq data, mRNA co-expression analysis revealed a strong positive correlation between SIRT5, MITF and two canonical MITF target genes, PPARGC1A and BCL2. Indeed, the correlation between SIRT5 and MITF expression was stronger than that of MITF with these two of MITF's targets (Figure 6F and S6C). As a specificity control, SIRT3 levels showed a modest, negative correlation with MITF expression (Figure S6C). These data suggest that SIRT5 expression levels influence expression of MITF and its targets in patient melanoma tumors.

Previous reports demonstrate that the proto-oncogene $c-M Y C$ is upregulated in melanoma tumors and cell lines, acting to bypass mutant BRAF- or NRAS-induced senescence during melanomagenesis (41). Furthermore, siRNA KD of c-MYC in melanocytic tumor cells results in a loss of MITF expression (68). Consistent with these data, we observed a loss of MITF expression and a concomitant reduction in expression of $c-M Y C$ in SIRT5-depleted melanoma cell lines. A positive correlation between SIRT5 and c-MYC RNA expression in melanoma tumors from TCGA data was observed (Figure S6C). Both c-MYC RNA and c-MYC protein levels were decreased in melanoma cells after SIRT5 ablation (Figure S6D and S6E).

Gene set enrichment analysis (GSEA) was used to identify pathways affected by SIRT5 depletion. GSEA revealed negatively enriched gene patterns in $c-M Y C$, c-MYC-target gene signatures, and mitochondrial biogenesis pathways (Figure S6F). We also observed a positive enrichment of genes involved in apoptosis, consistent with our observation that SIRT5 loss 
induces apoptosis in melanoma cells (see Figure 3). Ingenuity Pathway Analysis (IPA) of transcriptional regulators predicts that both MITF and $c-M Y C$ were significantly inhibited by SIRT5 depletion, based on comparisons between data from aggregated SIRT5 KD melanoma cells and SIRT5 control lines (Figure S6G). The multiple canonical pathways altered upon SIRT5 loss highlight other, pleiotropic effects of SIRT5 depletion on melanoma cells (Figure $\mathrm{S} 6 \mathrm{H})$. Taken together, these data show that SIRT5 promotes expression and activity of two key oncogenic drivers, MITF and c-MYC, in melanoma.

\section{SIRT5 regulates melanoma cell metabolism to promote histone acetylation}

To obtain systems-level insight into potential roles for SIRT5 in regulating gene expression, we re-analyzed our transcriptomic data, using a genome-scale model of human metabolism to identify metabolic reactions that change in activity after SIRT5 KD. The Recon1 human network model used contains a relationship between 3,744 reactions, 2,766 metabolites,1,496 metabolic genes, and 2,004 metabolic enzymes (69). This network model has been used successfully to predict the metabolic behavior of various cancer cells and stem cells $(70,71)$. Using this model, we identified a metabolic flux state most consistent with expression data for each of the three cell lines after SIRT5 depletion. This was achieved by maximizing the activity of reactions that are associated with up-regulated genes and minimizing flux through reactions that are downregulated for each condition, while simultaneously satisfying the stoichiometric and thermodynamic constraints embedded in the model (see Methods).

The model identified 20 reactions among the 3744 that showed significantly different activity across all cell lines after SIRT5 KD ( $p<0.01$; Figure 7A, Table S4). Among these, the enzyme ATP-Citrate Lyase (ACLY) was predicted to have the most significant change, with reduced activity after SIRT5 KD. ACLY generates acetyl-CoA from citrate, thereby playing an important role in supporting histone acetylation (72). Furthermore, the mitochondrial 
methylenetetrahydrofolate dehydrogenase reaction was also predicted to have reduced activity after SIRT5 loss, a part of the folate and one-carbon metabolism (1CM) pathways (see below). Several reactions involving cholesterol metabolism and nucleotide salvage were also affected by SIRT5 KD, highlighting the pervasive effects of SIRT5 in melanoma cells.

To test the predictions of the metabolic model, we evaluated protein acetylation levels in SIRT5 KD cells. Indeed, SIRT5 depletion induced a striking decrease in total lysine acetylation, most notably on histones, including H3K9 acetylation (H3K9ac) and H4K16 acetylation (H4K16ac) (Figure 7B and 7C). This reduction in $\mathrm{H} 3 \mathrm{~K} 9 \mathrm{ac}$, a known mark of active gene expression (73), combined with the decrease in MITF and c-MYC, prompted us to test whether H3K9ac levels are reduced within the promoter regions of these genes. CUT\&RUN (Cleavage Under Targets and Release Using Nuclease) followed by qRT-PCR in A2058 cells demonstrated that upon SIRT5 depletion, a significant reduction of H3K9ac in the promoter regions of both MITF and cMYC occurred (Figure 7D), suggesting a role for SIRT5 in maintaining transcriptional activity of these genes in melanoma cells by promoting histone acetylation.

After 4 weeks in culture following SIRT5 KD, a small residual population of A2058 cells overcame SIRT5 loss-of-function to survive and proliferate, although SIRT5 depletion was maintained. Importantly, total lysine acetylation and MITF expression was restored in surviving SIRT5 KD A2058 cell populations (Figure 7E and 7F), consistent with the relevance of SIRT5driven histone acetylation in melanoma survival. This phenotype was recapitulated in vivo. Although tumors that formed in SIRT5-deficient Braf ${ }^{C A} ; P_{t e n}^{f / f f l} ;$ Tyr::CreER mice were smaller than WT controls (see Figure 4), total lysine acetylation, H3K9ac, MITF and c-MYC protein levels were similar to controls. Markers for cell death (PARP cleavage) and cellular proliferation (PCNA and phospho-histone H3 S10 (H3pS10)) were also similar between SIRT5 WT and 
SIRT5-deficient tumors in the model, suggesting that these parameters may have recovered during successful tumor formation (Figure S7D).

Protein acetyltransferases employ acetyl-CoA to acetylate their protein targets, including histones (74). To investigate the potential basis for reduced histone acetylation in SIRT5depleted melanoma cells, we employed a sensitive mass spectrometry-based method to assess total cellular acetyl-CoA levels $(75,76)$. Surprisingly, we observed an increase of total cellular acetyl-CoA after SIRT5 KD (Figure 7G), implying that reduced acetyl-CoA levels do not contribute to the observed decrease in lysine acetylation upon SIRT5 depletion, and suggesting that other phenomena, such as reduced acetyltransferase activity may underlie the reduced acetylation levels in SIRT5-depleted melanoma cells (see Discussion).

\section{SIRT5 promotes one-carbon metabolism and histone methylation in melanoma}

To investigate further how SIRT5 may function to affect gene expression in melanoma, SIRT5depleted melanoma cell lines were profiled using liquid chromatography coupled tandem mass spectrometry (LC-MS/MS)-based metabolomics, followed by functional analysis using MetaboAnalyst pathway enrichment (Table S5). Two BRAF mutant lines (A2058 and A375) and an NRAS mutant (SK-MEL-2) showed perturbations in pathways involving 1CM in response to SIRT5 depletion (Figure 8A, S7A and S7B). 1CM is comprised of the linked folate and methionine cycles (77). Outputs include metabolites required for amino acid and nucleotide synthesis; glutathione for antioxidant defense; and crucially, S-adenosylmethionine (SAM) for methylation reactions, including those on histones. We observed a reduction in levels of several key $1 \mathrm{CM}$ metabolites upon SIRT5 depletion in BRAF mutant melanoma cell lines, but not in SKMEL-2 (Figure 8B). 
Histone methylation, particularly $\mathrm{H} 3 \mathrm{~K} 4$ trimethylation (H3K4me3), is highly sensitive to fluctuations in SAM levels (78). We observed reductions in H3K4me3 and H3K9me3 in melanoma cells following SIRT5 KD, consistent with 1CM perturbation (Figure 8C). However, addition of exogenous SAM did not consistently restore $\mathrm{H} 3 \mathrm{~K} 4 \mathrm{me} 3$ or $\mathrm{H} 3 \mathrm{~K} 9 \mathrm{me}$, nor did it markedly elevate levels of these marks in control cells (Figure S7C and not shown). As for acetylation, SIRT5-depleted melanoma cells that grew out after prolonged culture recovered H3K4me3 and H3K9me3 levels (Figure 8D), while maintaining reduced SIRT5 expression (Figure 7E), suggesting that loss of these histone modifications represents an important driver of the lethality associated with SIRT5 depletion in melanoma.

A decrease in cellular glutathione content occurring in the context of impaired 1CM would be predicted to elevate levels of cellular reactive oxygen species (ROS) (79). Consistently, in A2058 cells, we observed increased staining with 2',7'-dichlorofluorescin diacetate (DCFDA), a ROS-sensitive dye, following SIRT5 depletion (Figure 8E). However, treatment with the antioxidants, $\mathrm{N}$-acetylcysteine, mitoTEMPOL or $\beta$-mercaptoethanol failed to mitigate cell lethality after SIRT5 loss (data not shown), indicating that regulation of ROS levels is not likely a primary determinant of the requirement of melanoma cells for SIRT5.

We noted that previous proteomic surveys identified the $1 \mathrm{CM}$ enzyme, MTHFD1L (methylenetetrahydrofolate dehydrogenase (NADP ${ }^{+}$dependent) 1 like), as a candidate SIRT5 substrate $(11,80)$. MTHFD1L is a $1 \mathrm{CM}$ enzyme that participates in the folate cycle to convert formate and tetrahydrofolate into 10-formyl-tetrahydrofolate in an ATP-dependent reaction. We tested the interaction of MTHFD1L with SIRT5 in the context of melanoma, and found that MTHFD1L co-immunoprecipitates with SIRT5 (Figure 8F). These data suggest a potential role for SIRT5 in regulating multiple 1CM enzymes, such as SHMT2 and potentially MTHFD1L and others, to promote $1 \mathrm{CM}$ and histone methylation. Likewise, since SK-MEL-2 cells showed a 
reduction in histone H3K4me3 levels without apparent declines in $1 \mathrm{C}$ metabolites under our experimental conditions, it is likely that SIRT5 plays additional roles in regulating histone methylation, perhaps in an oncogenic driver-dependent manner. We propose that SIRT5 regulates histone methylation and acetylation via regulation of multiple protein targets in melanoma cells. 


\section{Discussion}

Sirtuin-family $\mathrm{NAD}^{+}$-dependent protein deacylases regulate metabolism and other diverse aspects of cell biology (81). SIRT5 is a poorly-understood, atypical sirtuin, whose primary known biochemical function is to remove succinyl, malonyl, and glutaryl groups from lysines on its target proteins $(8,9,11-13)$. A substantial fraction of SIRT5 is present in the mitochondrial matrix; however, SIRT5 is present and functional in the cytosol, and even in the nucleus (11, 14). Most of the phenotypes associated with SIRT5 loss-of-function in normal cells and tissues reported in the literature to date are remarkably mild (17). In sharp contrast, here we report that cutaneous and uveal melanoma cells show exquisite dependency on SIRT5, in a genotypeindependent manner. SIRT5 depletion, induced by shRNA or CRISPR/Cas9, provokes dramatic, rapid loss of cell viability and induction of apoptosis in both cutaneous and uveal melanoma cell lines. Likewise, SIRT5 promotes melanoma xenograft tumor formation in immunocompromised mice, and melanoma formation in an autochthonous Braf; Pten-driven mouse melanoma strain.

Our transcriptomic analyses reveal that SIRT5 plays a major role in maintaining proper gene expression in melanoma cells. SIRT5-dependent genes notably include the lineage-specific oncogenic transcription factor MITF (82) and c-MYC (41). In the TCGA dataset, SIRT5 levels correlate with those of MITF and c-MYC, suggesting that SIRT5 activity influences both MITF and $c-M Y C$ expression in a physiologic context. Indeed, we found that SIRT5 depletion results in loss of $\mathrm{H} 3 \mathrm{~K} 9 \mathrm{ac}$, a marker for active transcription, within the promoter regions of these genes. These data are consistent with previously published results describing a role for histone modifications in sustaining MITF expression and melanoma proliferation (83). Genetic or pharmaceutical inhibition of the p300 acetyltransferase results in reduced MITF expression, reduced histone acetylation within of the MITF promoter, and induction of markers of cellular 
senescence in melanoma cell lines, suggesting regulation of chromatin dynamics as a mechanism of MITF expression and melanoma growth (83). Via metabolomic analysis, we identified a role for SIRT5 in promoting 1CM in two BRAF-dependent cell lines, and in maintaining histone trimethylation at $\mathrm{H} 3 \mathrm{~K} 4$ and $\mathrm{H} 3 \mathrm{~K} 9$, marks associated with transcriptional activation and repression, respectively. SIRT5 also plays a distinct role in maintaining histone acetylation. To our knowledge, SIRT5 is the first protein implicated in maintaining both histone methylation and acetylation, highlighting its important roles in maintaining chromatin structure and gene expression in melanoma.

Our in vivo findings in an autochthonous system are in contrast to a published study by Moon et al., in which SIRT5 deficiency was found to exert no impact on tumor growth in a similar mouse melanoma model as the one used in our studies (84). Several potential explanations exist for this discrepancy. Moon et al. used a Sirt5 allele distinct from the one employed in our work; the Sirt5 allele used in their analysis deletes a single exon in the Sirt5 gene (16), whereas the one used herein deletes essentially the entire Sirt5 protein coding sequence (15). Likewise, subtle genetic background differences in the strains of the mice used may contribute to these discrepancies, as could microbiome differences between the mouse colonies. Another potential explanation involves the protocol used to induce gene recombination; we applied a higher concentration of tamoxifen than did Moon et al. (64.5 mM versus $5 \mathrm{mM})$. Importantly, since this model is a global Sirt5 KO, we cannot rule out the possibility that SIRT5 may function melanoma cell non-autonomously in this system, for example, by modulating the anti-melanoma immune response or other aspects of the tumor microenvironment. However given the striking dependency of cultured melanoma cells on SIRT5 in vitro, we strongly suspect that a very important component of SIRT5's function, at minimum, is a cell-autonomous pro-survival role in melanoma cells. 
MITF is a member of microphthalmia family of transcription factors, and is dysregulated in melanoma (85). Attenuation of melanocyte differentiation and pigmentation are observed in humans and mice deficient for MITF activity, highlighting the importance of MITF in melanocyte survival and function. Likewise, MITF is known to play key roles in melanoma cell survival and differentiation, and MITF amplification occurs in $15 \%$ to $20 \%$ of melanomas, associated with a worsened prognosis (64). In melanoma cell lines where MITF is expressed, SIRT5 depletion induced a rapid decrease in expression of MITF itself and several well-characterized MITF targets. Likewise, in TCGA data, SIRT5 and MITF levels were highly correlated, suggesting that SIRT5 may play a role in regulating MITF in tumors in vivo. Notably, we were unable to rescue the lethality of SIRT5 depletion by overexpressing MITF in melanoma cells (data not shown); however, this experiment is complicated by the fact that MITF overexpression itself can drive melanoma cells to leave the cell cycle and differentiate, and thus is likely selected against in short-term culture (86). Likewise, we were unable to rescue SIRT5-depleted melanoma cells via c-MYC overexpression, although we were able to overexpress c-MYC (data not shown). Nevertheless, given the well-known importance of these transcription factors in melanoma pathobiology, we hypothesize that loss of MITF and c-MYC expression likely represent important mechanisms through which SIRT5 promotes melanoma viability.

We did not observe major effects of SIRT5 depletion on OCR, ECAR, or overall ATP production in melanoma. Instead, through mass spectrometry-based metabolite profiling, we identified onecarbon metabolism (1CM) as one SIRT5 target pathway likely important for maintenance of gene expression and melanoma viability. 1CM consists of the linked folate and methionine cycles. A major output of $1 \mathrm{CM}$ is SAM, the universal methyl donor in mammalian cells. Metabolite profiling in two BRAF mutant melanoma cells lacking SIRT5 reveals profound perturbations in levels of many $1 \mathrm{C}$ metabolites, including reductions in cellular SAM. Moreover, H3K4me3, a mark of active gene expression and a sensitive marker for intracellular SAM levels, 
drops in response to SIRT5 loss-of-function. Furthermore, global lysine acetylation and H3K9me3, which marks heterochromatic regions in the genome (87) decrease upon SIRT5 loss. Likewise, oxidative stress increases in SIRT5-depleted melanoma cells, consistent with impaired regeneration of reduced glutathione, a major antioxidant species and an output of $1 \mathrm{CM}$.

Many open questions remain as to the mechanisms by which SIRT5 promotes proper gene expression and viability in melanoma. The accumulation of acetyl-CoA in SIRT5-depleted melanoma cells suggests that SIRT5 may promote the activity of a histone acetyltransferase to promote histone acetylation, a possibility that we are currently investigating. Alternatively, SIRT5 could promote generation of a localized acetyl-CoA pool necessary to drive histone acetylation (cf. the nuclear pool (74)), without influencing global acetyl-CoA levels. A large number of studies implicate alterations in levels of specific metabolites in driving chromatin modifications (88). Increased lactate, for example inhibits histone deacetylases, thereby increasing histone acetylation (89). Although we observe only modest and, in some cases, inconsistent changes in cellular metabolite levels upon SIRT5 KD, it is possible that alterations in levels of specific metabolites, or a combination of these metabolite abnormalities, may in part be responsible for the loss of histone modifications we observe. In addition, we identified MTHFD1L as a novel SIRT5 interactor and candidate target that may play a role in SIRT5-mediated regulation of $1 \mathrm{CM}$. Unfortunately, we have been unsuccessful at rescuing the cellular lethality associated with SIRT5 depletion using relevant small molecule metabolites or drugs (acetate, acetyl-CoA, SAM, serine, glycine, histone deacetylase and demethylase inhibitors, antioxidants, nucleotides, and amino acids [data not shown]). We suspect that this reflects pleiotropic functions and targets of SIRT5 in melanoma cells, impairment of which cannot be rescued by intervention in any individual pathway. SIRT5 targets involved in other pathways -- e.g. ROS suppression, cell 
death $(32,90)$, and others -- could well contribute to the requirement of melanoma cells for SIRT5. Likewise, we identified perturbations in innate immune pathways in SIRT5-depleted melanoma cells, which could also contribute to the requirement of melanoma cells for this protein. This is consistent with the hundreds of cellular targets of SIRT5, involved in diverse cellular pathways, identified in proteomics studies (17). Moreover, it is consistent with the observation that SIRT5 plays pro-survival roles across multiple different cancer types, via distinct proposed mechanisms. As the dominant cellular desuccinylase/demalonylase/deglutarylase, it is possible that SIRT5 is recruited to play distinct roles in supporting tumorigenesis, modulating activities of different suites of targets and pathways, in a cancer type-specific manner.

Overall, our data reveal a major, hitherto unknown requirement for SIRT5 in melanoma cell survival, through suppression of apoptosis via regulation chromatin modifications and expression of critical pro-survival genes, including MITF and c-MYC (Figure 8G). These results, along with those already in the literature (7), suggest that SIRT5 may play potent oncogenic roles across many diverse tumor types, seemingly engaging a variety of different cellular mechanisms to do so in a cancer- and context-specific manner. Since the phenotypes of Sirt5 null mice are quite mild, we propose that SIRT5 may represent an attractive new therapeutic target, in melanoma and specific other cancer types. In this regard, published studies (17, 9194), including recent work focused on breast cancer (37) demonstrate that SIRT5 is in principle druggable with small molecules. SIRT5 dependency may be particularly translationally significant in uveal melanoma, where currently no effective therapeutic options exist for patients with metastatic disease. 


\section{Materials and Methods}

Analysis of SIRT5 gene amplification, mRNA and protein expression in melanoma

Percentages of genetic alterations, as indicated, in SIRT1-SIRT5, BRAF, NRAS, PTEN, MITF and NF1 in human melanoma cases were calculated from TCGA data where copy number and mutation data were available ( $n=287$, Provisional, analyzed on cBioPortal). Kaplan-Meier analysis of overall or disease-free survival in melanoma patients with or without copy number gain or amplification of SIRT5 were similarly analyzed. Sirtuin copy number analysis of melanoma cell lines was performed by high density SNP array of 8 primary, 72 stage III, 51 stage IV and 8 stage III/IV (metastatic disease) melanoma cell lines, as previously described $(48,49)$. Data were analyzed using Nexus Copy Number (BioDiscovery) for copy gain, copy loss and loss of heterozygosity of genes and chromosomal regions. Data from the TCGA was used via cBioPortal to further investigate SIRT5 in melanoma $(n=478$; Cancer Genome Atlas Network), including copy number (by GISTIC 2.0) and expression data (by RNAseq) and correlation with clinical attributes, including the Clark level at diagnosis of the melanoma.

\section{Immunohistochemical analysis of SIRT5 in human nevus and melanoma}

The study was undertaken with Human Ethics Review Committee approval and patient's informed consent. The Melanoma Institute Australia Medical Research Database and archival files of the Department of Tissue Pathology and Diagnostic Oncology, Royal Prince Alfred Hospital, were utilized to identify melanocytic lesions (human ethics committee approval X110289, HREC/11/RPAH/444). Melanocytic lesions were selected based on the pathological diagnosis of the melanocytic lesion as a compound nevus, dysplastic nevus, thin primary melanoma (Breslow thickness $<1 \mathrm{~mm}$ ), thick primary melanoma (Breslow thickness $>1 \mathrm{~mm}$ ) or metastatic melanoma in regional lymph node or distant metastatic site. Immunohistochemical SIRT5 staining intensity in melanocytes was scored by two pathologists blinded as to the diagnosis associated with each tissue core. Scores were then averaged. 


\section{Cell culture}

Cutaneous melanoma cell lines with mutations in either NRAS or BRAF: A375, A2058, SK-MEL2, SK-MEL-28, VMM15, and VMM917 were purchased from ATCC (Table S2); SK-MEL-19 was generously provided by Monique Verhaegen (UM); C8161 was provided by Dr. Zaneta Nikolovska-Coleska (UM); SK-MEL-239 and vemurafenib-resistant (SK-MEL-239VR) lines were generously provided by Dr. Emily Bernstein (ISMMS). Uveal melanoma cell lines MP-38, MP-41 and MP-46 were purchased from ATCC; cell line 92-1 was purchased from Sigma-Aldrich (Table S2). Ovarian cancer cell lines A2780, SK-OV-3, OVCAR4 and OVCAR10 were generously provided by Kathleen Cho (UM).

Unless otherwise noted, A375, A2058 and SK-MEL-19 cell lines were cultured in DMEM (Gibco) containing $4.5 \mathrm{~g} / \mathrm{L}$ glucose, $110 \mathrm{mg} / \mathrm{L}$ sodium pyruvate, $4 \mathrm{mM} \mathrm{L}-$ glutamine, $100 \mathrm{units} / \mathrm{mL}$ penicillin, $100 \mu \mathrm{g} / \mathrm{mL}$ streptomycin and $10 \%$ heat-inactivated FBS. SK-MEL-2 and SK-MEL-28 cell lines were cultured in MEM (Gibco) containing 1.0g/L glucose, $110 \mathrm{mg} / \mathrm{L}$ sodium pyruvate, $2 \mathrm{mM} \mathrm{L-}$ glutamine, $0.1 \mathrm{mM}$ non-essential amino acids (Gibco), 100units $/ \mathrm{mL}$ penicillin, $100 \mu \mathrm{g} / \mathrm{mL}$ streptomycin solution and 10\% heat-inactivated FBS. VMM15, VMM917, SK-MEL-239 and SKMEL-239VR cell lines were cultured in RPMI (Gibco) containing 4.5g/L glucose, 10mM HEPES, $110 \mathrm{mg} / \mathrm{L}$ sodium pyruvate, $4 \mathrm{mM}$ L-glutamine, $100 \mathrm{units} / \mathrm{mL}$ penicillin, $100 \mu \mathrm{g} / \mathrm{mL}$ streptomycin and $10 \%$ heat-inactivated FBS. SK-MEL-239VR derivative cells were cultured in $2 \mu \mathrm{M}$ vemurafenib (Cayman Chemical). The C8161 cell line was grown in DMEM/F12 (1:1) (Gibco) containing 1.2mM L-glutamine, $0.1 \mathrm{mM}$ non-essential amino acids, 100 units $/ \mathrm{mL}$ penicillin, $100 \mu \mathrm{g} / \mathrm{mL}$ streptomycin and 5\% heat-inactivated FBS. MP-38, MP-41 and MP-46 cells were cultured in RPMI (Gibco) containing 2 mM L-glutamine, 10 mM HEPES, 110mg/L sodium pyruvate, $4.5 \mathrm{~g} / \mathrm{L}$ glucose, $100 \mathrm{units} / \mathrm{mL}$ penicillin, $100 \mu \mathrm{g} / \mathrm{mL}$ streptomycin and $25 \%$ heatinactivated FBS; 92-1 cells were cultured in RPMI (Gibco) containing 2 mM L-glutamine, $10 \mathrm{mM}$ 
HEPES, $110 \mathrm{mg} / \mathrm{L}$ sodium pyruvate, $4.5 \mathrm{~g} / \mathrm{L}$ glucose, $100 \mathrm{units} / \mathrm{mL}$ penicillin, $100 \mu \mathrm{g} / \mathrm{mL}$ streptomycin and 10\% heat-inactivated FBS. S-adenosylmethionine (SAM) resuspended in $0.005 \mathrm{M}$ sulfuric acid and $10 \%$ ethanol was purchased from New England Biolabs. All cell lines were routinely confirmed to be free of mycoplasma contamination by PCR assay and were grown in a humidified chamber at $37^{\circ} \mathrm{C}$ containing $5 \% \mathrm{CO}_{2}$. All cutaneous melanoma cell lines were authenticated via STR profiling at the University of Michigan DNA Sequencing Core (data available upon request). Table S2 lists the genetic alteration of each cell line, and the sex and age of the patient at the time of cell line derivation, if known. Ovarian cancer cell lines A2780, OVCAR10, OVCAR4 were cultured in RPMI (Gibco) containing 2 mM L-glutamine, $10 \mathrm{mM}$ HEPES, $110 \mathrm{mg} / \mathrm{L}$ sodium pyruvate, $4.5 \mathrm{~g} / \mathrm{L}$ glucose, $100 \mathrm{units} / \mathrm{mL}$ penicillin, $100 \mu \mathrm{g} / \mathrm{mL}$ streptomycin and 10\% heat-inactivated FBS. SK-OV-3 cells were cultured in McCoy's (Gibco) medium supplemented with $100 \mathrm{units} / \mathrm{mL}$ penicillin, $100 \mu \mathrm{g} / \mathrm{mL}$ streptomycin and $10 \%$ heatinactivated FBS.

\section{Lentiviral transduction}

Lentiviral plasmids in the pLKO.1 backbone, encoding puromycin $\mathrm{N}$-acetyl-transferase, containing shRNAs targeting human SIRT5 or murine Sirt5 (Table S6) were used to generate high-titer lentiviral particles at the Vector Core (UM). A non-silencing control shRNA against Gaussia luciferase was used as the non-targeting control (NT). KD3 and KD4 shRNA lentiviral plasmids targeting human SIRT5 were designed based on previously published CRISPR screens $(95,96)$. All other shRNA plasmids were purchased through the Vector Core (UM). Lentiviral transduction was carried out in the presence of $8 \mu \mathrm{g} / \mathrm{ml}$ polybrene in complete growth medium for $24 \mathrm{hrs}$, , after which medium was replaced with fresh complete growth medium. Puromycin was added to a final concentration of $1 \mu \mathrm{g} / \mathrm{ml} 48 \mathrm{hrs}$. post-transduction to select for transductants. Successful SIRT5 KD was routinely confirmed by western blotting 48-96 hrs. post-transduction. 


\section{Proliferation assays}

Forty-eight hrs. after lentiviral transduction, $5 \times 10^{3}$ cells were plated into 96 -well plates in the presence of $1 \mu \mathrm{g} / \mathrm{ml}$ puromycin or in $1 \mu \mathrm{g} / \mathrm{ml}$ puromycin with a cocktail of $0.1 \mathrm{mM}$ non-essential amino acids (Gibco) and 5mM alpha-ketoglutarate (Sigma), where indicated. Twenty-four hrs. after plating, relative cell mass was assessed using WST-1 Cell Proliferation Reagent (Clontech) per manufacturer's instruction. After addition of WST-1, plates were incubated at $37^{\circ} \mathrm{C}$ for $2 \mathrm{hrs}$. before reading the optical density at 450nm. OD450nm was assessed every 24 hrs. as indicated and plotted.

Ovarian cancer cell lines, A2780, SK-OV-3, OVCAR10 and OVCAR4, were transduced with lentiviral particles carrying either a non-silencing control shRNA against Gaussia luciferase, KD1 or KD2 shRNA targeting human SIRT5. Lentiviral transduction was carried out in the presence of $8 \mu \mathrm{g} / \mathrm{ml}$ polybrene in complete growth medium for $24 \mathrm{hrs}$., after which medium was replaced

with fresh complete growth medium. Puromycin was added to a final concentration of $2 \mu \mathrm{g} / \mathrm{ml} 48$ hrs. post-transduction to select for transductants. Successful SIRT5 KD was confirmed by western blotting. Following puromycin selection and confirmation of SIRT5 KD, 2.5x10 4 cells were plated in complete growth media in triplicates into 12-well plates. Cell number in each well were assessed every $24 \mathrm{hrs}$. after plating by manual counting using a hemocytometer.

\section{Colony formation assays}

Two million A2058 or SK-MEL-2 melanoma cell lines were lentivirally transduced in a 10-cm dish with a non-silencing shRNA or one of two shRNAs targeting human SIRT5. Forty-eight hrs. post-transduction $1 \times 10^{5}$ cells were plated into each of four wells of a six-well dish as previously described. Twelve days after transduction, puromycin-selected cells were stained with $0.25 \%$ (w/v) crystal violet (Sigma) in $20 \%$ ethanol for 30 minutes according to standard protocols. 
Colony formation was quantified by solubilizing crystal violet in a $30 \%$ methanol, $10 \%$ acetic acid solution and absorbance measured at OD590nm.

\section{CRISPR/Cas9 targeting of SIRT5}

CRISPR plasmids were generated as described (97). Briefly, guide sequences (Table S6) targeting the SIRT5 locus were inserted into pSpCas9(BB)-2A-Puro (PX459) backbone (Addgene catalog \#62988). Guide sequences were designed based on previous CRISPR screens $(95,96,98)$. Sanger sequencing confirmed successful cloning of guide sequences. A2058 cells were seeded at 50,000 cells/well two days prior to transfecting 500 ng of each indicated plasmid using polyethylenimine (PEI) in DMEM without serum or antibiotics.

Transfected cells were selected using puromycin $(1 \mu \mathrm{g} / \mathrm{ml})$ for 7 days, beginning 24 hrs. posttransfection. Fourteen days after transfection, cells were fixed and stained with $0.25 \%$ crystal violet in $20 \%$ ethanol for 30 mins at room temperature. Colony formation was quantified by solubilizing crystal violet in a $30 \%$ methanol, $10 \%$ acetic acid solution and absorbance measured at OD590nm. Confirmation of SIRT5 KD via immunoblot was performed 33 days post-transfection.

\section{Immunoblotting}

Whole-cell protein extracts were prepared in protein sample buffer $(62.5 \mathrm{mM}$ Tris $\mathrm{pH} 6.8,2 \%$ SDS, $10 \%$ glycerol). Lysates were sonicated for 30 seconds using a Branson Sonifier set to output "2." Lysates were then clarified by centrifugation at $15,000 \mathrm{rcf}$ for 30 minutes at $4^{\circ} \mathrm{C}$. Protein concentrations were determined using DC Protein Assay (Bio-Rad). Equivalent amounts $(10-50 \mu \mathrm{g})$ of total protein, supplemented with $710 \mathrm{mM} \beta$-mercaptoethanol and $0.01 \%(\mathrm{w} / \mathrm{v})$ bromophenol blue, then boiled for 5 minutes, were fractionated by SDS-PAGE on a $12 \%$ or $15 \%$ polyacrylamide gel, electrophoretically transferred to PVDF, and probed with antibodies diluted in 5\% nonfat milk in 1XTBS-0.1\% Tween-20. Membranes were imaged on an ImageQuant LAS 
4000 Scanner (GE Healthcare) after application of Immobilon Western HRP Substrate (Millipore). See Table S7 for antibodies used in this study.

\section{Immunofluorescence}

A2058 cells were plated at a density of $0.5 \times 10^{6}$ per well in six-well plates containing glass coverslips (Werner \#1.5 thickness, 12mm diameter). Twenty-four hrs. later, cells were transduced with either a lentivirus expressing a non-silencing shRNA (control) or one of two shRNAs targeting SIRT5 (KD1 or KD2). Ninety-six hrs. after transduction, puromycin-selected cells were incubated with 100nM Mitotracker (BD Biosciences) for 30 minutes at $37^{\circ} \mathrm{C}$, washed with PBS, and fixed in 3.7\% formaldehyde. After permeabilization in $0.3 \%$ Triton X-100 for 10 minutes at room temperature, cells were blocked with $5 \%$ normal goat serum in $0.2 \%$ Triton $X$ 100 in PBS for one hour. Cells were incubated in SIRT5 (Sigma) primary antibody diluted to 1 $\mu \mathrm{g} / \mathrm{ml}$ in $5 \%$ bovine serum albumin in $0.2 \%$ Triton $\mathrm{X}-100$ in PBS overnight at $4^{\circ} \mathrm{C}$. Cells were then washed 3 times in 1X PBS and incubated in Alexa 488 anti-rabbit (Invitrogen) diluted $1: 1000$ in $5 \%$ bovine serum albumin in $0.2 \%$ Triton X-100 in PBS for 1 hour at room temperature. Cells were washed 2 times in 1X PBS, and 1X with PBS supplemented with 0.01 $\mu \mathrm{g} / \mathrm{ml}$ DAPI. Cells were mounted with prolong gold antifade reagent (ThermoFisher) and imaged on an Olympus FV 500 Confocal microscope.

\section{Cellular Fractionation}

Lentivirally-transduced A2058 cells were washed twice with 1X PBS and harvested by scraping 96hrs. post-transduction. Subcellular fractionation was carried out using the CelLytic NuCLEAR Extraction Kit (Sigma-Aldrich), according to the manufacturer's protocol. The cytoplasmic fraction was concentrated using Amicon Ultra $0.5 \mathrm{~mL}$ Centrifugal Filters (Sigma-Aldrich), according to manufacturer's instructions. Each fraction and whole-cell pellets were resuspended in protein sample buffer, processed as previously (see above), and analyzed by immunoblot. 


\section{Annexin V flow cytometry}

A2058 and SK-MEL-2 cells were plated at a density of $0.5 \times 10^{6}$ per well of two six-well plates/cell line. Four wells of each cell line were transduced with either a lentivirus expressing a nonsilencing shRNA (control) or one of two shRNAs targeting SIRT5 (KD1 or KD2). Ninety-six hrs. after transduction, puromycin-unselected cells were harvested and stained for 30 minutes at room temperature with FITC Annexin V (BD Biosciences) and propidium iodide (Sigma), according to the BD Biosciences staining protocol. Cells were analyzed by flow cytometry using a BD FACSCalibur and results were plotted using FlowJo 10.2 analysis software.

\section{Xenograft assays}

A2058 puromycin-unselected cells were harvested $72 \mathrm{hrs}$. post-transduction with pLKO control, pLKO SIRT5 KD1, or pLKO SIRT5 KD2. Subcutaneous tumor growth was initiated by injection of $1 \times 10^{6}$ cells of each cell line, resuspended in 1:1 DMEM:Matrigel Matrix (Corning), into the contralateral flanks of 11-13 week old NOD.Cg-Prkdc scid $H r^{h r} / \mathrm{NCrHsd}$ female mice (Envigo). Each experimental group contained 5 mice. Tumor size was measured in millimeters (mm) using Vernier calipers at the timepoints indicated. Tumor volume was calculated according to the formula:

$$
V=\frac{X\left(Y^{2}\right)}{2}
$$

where $V$ is tumor volume in $\mathrm{mm}^{3}, X$ is the longest length of the tumor, and $Y$ is the shortest length of the tumor, perpendicular to $X(99,100)$. All mice were euthanized when a tumor ulcerated or reached $2000 \mathrm{~mm}^{3}$. All mice were housed at the Biomedical Science Research Building (UM). All vertebrate animal experiments were approved by and performed in accordance with the regulations of the University Committee on Use and Care of Animals. 


\section{Tumor induction in Braf ${ }^{C A} ;$ Pten $^{f / f t} ;$ Tyr::CreER;Sirt5 mice}

Sirt5 KO mice (15) were crossed with Braf ${ }^{C A} ; P_{t e n}^{f / / f l} ;$ Tyr::CreER mice (55) to generate groups of littermate Braf ${ }^{C A} ; P^{A} n^{f l / f l} ;$ Tyr::CreER;Sirt5 KO and WT controls on a mixed BL/6/129SvJ genetic background. For melanoma induction, flanks of adult mice (4-9 weeks of age) were treated with depilatory cream with a cotton applicator. After 3-5 minutes, the area was rinsed with distilled water, and the treatment spot marked. Topical administration of 4-hydroxytamoxifen (4-HT; $25 \mathrm{mg} / \mathrm{ml}$ in DMSO, Cayman Chemical) was repeated on each of three consecutive days, by applying one microliter of 4-HT to the spot. Mice were monitored for tumor appearance, which occurred typically within 4-8 weeks, and euthanized when a tumor ulcerated or reached $2000 \mathrm{~mm}^{3}$. All mice were housed at the Biomedical Science Research Building (UM). Experiments were approved by and performed in accordance with the regulations of the University Committee on Use and Care of Animals.

\section{Transcriptomic analysis of SIRT5 depletion}

A2058, A375 and SK-MEL-2 cells were plated at a density of $0.5 \times 10^{6} /$ well of two six-well plates per cell line. Four wells of each cell line were transduced with either a lentivirus expressing a non-silencing shRNA (control) or one of two shRNAs targeting SIRT5 (KD1 or KD2). Total RNA was extracted in TRIzol (Invitrogen) 96 hrs. post-transduction from three wells and treated with RNAse-free DNAse I (Roche) for 1 hour at $37^{\circ} \mathrm{C}$, according to manufacturer's instructions. RNA samples ( $n=3$ biological replicates for each of 3 cell lines for each condition) were submitted to the University of Michigan Sequencing Core for sample processing and Illumina HiSeq-4000 50nt paired-end sequencing. The remaining well was harvested for protein to confirm SIRT5 KD by immunoblot. Illumina libraries were prepared using random primers according to manufacturer's instructions. 
Paired-end reads were assessed for various quality metrics before and after trimming via FastQC v.0.11.9 (101) and MultiQC v.1.9 (102). All quality control modules investigated suggested high sample quality and removal of adapter content. Reads were trimmed by Trimmomatic v.0.39 (103) in paired-end mode, yielding filtered R1 and R2 FASTQ files containing only mate-paired reads. Trimmed FASTQ files were aligned by STAR v.2.7.3a (104) in paired-end mode against Gencode (GRCh38 Release 34 primary assembly). For each unique sample, all corresponding FASTQ files (i.e. R1/R2, split lanes) were aligned together in a single STAR call via -readFilesIn to yield single sorted BAM files for each sample. STAR-output BAM files were subsequently counted by the SubRead v.2.0.0 (105) featureCounts command to yield counts. Count matrices produced by featureCounts were then supplied to the Bioconductor package DESeq2 v.1.28.1 (106) for differential gene expression analysis. Contrasts controlling for cell line and pooling unique knockdowns together were utilized to discern the generalized impacts of SIRT5 knockdown. DESeq2 results were first independently filtered, and then the unique, non-censored genes across all contrasts of interest were combined to generate a standardized list of genes that were re-evaluated for differential expression without independent filtering to ensure gene-level statistics were generated for every contrast and not missing. All log2(Fold Change) values were subsequently shrunk, utilizing the apeglm (v.1.10.0) package's “Approximate Posterior Estimation for GLM” (apeglm) algorithm (107), providing Bayesian shrinkage estimators for the effect size. Genes with calculated p-values, after adjusting for multiple comparisons by the Benjamini-Hochberg method (i.e. q-values), at $p<0.01$ (5\% FDR) and a $\mid \log 2($ Fold Change)|>1 were then considered significant. FPKM values were estimated using DESeq2 FPKM function in robust size factor normalization mode.

\section{Differently expressed gene correlation with SIRT5 expression in human melanoma} TCGA RNA data (EBPlusPlusAdjustPANCAN_IIluminaHiSeq_RNASeqV2.geneExp.tsv) were downloaded from the Pan-Cancer Atlas site (PanCanAtlas; https://gdc.cancer.gov/about- 
data/publications/pancanatlas; accessed November, 2020, (108)). These data were filtered to encompass 474 sequenced human skin cutaneous melanoma samples with RNA abundance data (i.e. counts). The counts of differently expressed genes (DEGs) in response to aggregate SIRT5 KD $(q<0.05)$ that uniquely mapped to Entrez Ids were correlated with SIRT5 counts in these clinical sample via Spearman's rank correlation. Genes of interest were identified by overlapping genes evaluated for correlation with known oncogenes from the ONGene database ((109); http://ongene.bioinfo-minzhao.org/) and melanoma subtype signatures defined

previously (110). Prior to intersection, the melanoma subtype signatures (gene symbols) were updated using the HGNC Multi-symbol checker (https://www.genenames.org/tools/multi-symbolchecker/) to maximize the number of genes overlapping those in the more recent genome annotation we utilized.

\section{Code Availability}

Supporting analysis code, files, and analysis documentation is hosted at https://github.com/monovich/giblin-sirt5-melanoma.

\section{SIRT5 RNA-seq gene ontology}

Differentially expressed genes were classified by a padj-value $<0.05$. To identify shared or unique protein-coding genes between A2038, A375, and SK-MEL-2 datasets, differentially expressed protein-coding genes from each cell line were first designated as up- or downregulated upon SIRT5 KD. Each up- or down-regulated protein-coding list was then crossreferenced between the three cell lines to generate the values indicated in the Venn diagram. Gene set enrichment analysis (GSEA (111)) was performed using the Preranked function in the GSEA 4.1.0 software against the MSigDB 7.2 release (112) on the unfiltered RNAseq dataset of pooled SIRT5 KD against pooled control samples (GSEA references here). The list of ranked genes used as input for GSEA analysis was generated by multiplying the sign of fold-change (+ 
or -) with - $\log ($ padj). Additional gene ontology was performed using Ingenuity Pathway Analysis (IPA: QIAGEN Inc.) on transcripts with a padj-value<0.05 from the RNAseq dataset of pooled SIRT5 KD against pooled control samples. All pathways with a p-value $<0.05(-\log (p-v a l u e)>1.3)$ were deemed significant. As defined by IPA, significant upstream regulators were defined as significant based on a p-value $<0.05$ and an absolute activation z-score greater than 2 .

\section{CUT\&RUN}

CUT\&RUN for H3K9ac in A2058 cells was carried out using the CUT\&RUN Assay Kit (Cell Signaling Technology), according to the manufacturer's instructions. Briefly, $2 \times 10^{5} \mathrm{~A} 2058$ cells were harvested $96 \mathrm{hrs}$. after transduction with either a lentivirus expressing a non-silencing shRNA (control) or one of two shRNAs targeting SIRT5 (KD1 or KD2) and subjected to chromatin immunoprecipitation using $5 \mu \mathrm{l}$ of either anti-H3K9ac or, as a negative control, rabbit IgG isotype antibody. Samples were incubated overnight with shaking at $4^{\circ} \mathrm{C}$. DNA was then extracted via ethanol precipitation overnight at $-20^{\circ} \mathrm{C}$, and then resuspended in $50 \mu \mathrm{l}$ of water. The purified DNA (2 $\mu$ l per reaction) was quantified by real-time PCR using SimpleChIP human MITF (Cell Signaling Technology) or human c-MYC primers (forward: 5'-

GGACCCGCTTCTCTGAAAGG-3' and reverse: 5'-GCAAGTGGACTTCGGTGCTTACC-3', as previously described (113)), and SYBR Select Master Mix (Applied Biosystems). Ct values were normalized to control H3K9ac values. ChIP-qRTPCR was done three independent times with $\mathrm{n}=3$ technical replicates each time.

\section{Identifying differentially active reactions using genome-scale metabolic modeling} Gene expression data from the melanoma cell lines A2058, A375, and SK-MEL-2 was used as input to identify differentially active reactions in the human genome-scale metabolic model using a modeling approach detailed in Shen et al. $(114,115)$. This approach identifies a metabolic flux state that best fits the transcriptomics profile in each condition. Normalized expression data for 
each cell line was compared between the two groups ( $C$ vs. KD) to attain a list of significantly up- and down-regulated genes using a significance threshold of $p<0.05$ and a z-score threshold above 2 or below -2 , respectively. These genes were then overlaid onto the metabolic model based on gene-protein-reaction annotations in the model. Finally, reaction flux data was generated using a linear optimization version of the iMAT algorithm $(71,115)$ with the following inputs: the RECON1 model, the list of up- and down-regulated genes, and the recommended values for the optional parameters (rho $=1 \mathrm{E}-1$, kappa $=1 \mathrm{E}-1$, epsilon $=1$, ode $=0$ ). This resulted in flux predictions for all three cell lines. Differentially active reactions were identified by transforming the fluxes into z-scores and estimating the significance of the difference between Z-scores before and after KD across all cell lines using a t-test. The flux data for all the reactions is available in Table S4. Flux data was visualized through heatmaps generated using $R$ software, where reactions were clustered by rank correlation. Only reactions with significant flux difference between control and KD groups $(p<0.01)$ are shown. Transport reactions were excluded from the analysis.

\section{Acetyl-CoA Quantification}

Acetyl-CoA were quantified by stable isotope dilution liquid chromatography-high resolution mass spectrometry, as previously described $(75,76)$. Cell pellets were spiked with an internal standard prepared as described (75), and sonicated for 12 cycles of 0.5 sec. pulses in $10 \%$ $(w / v)$ trichloroacetic acid (Sigma Aldrich) in water. Protein was pelleted by centrifugation at $17,000 \mathrm{rcf}$ for $10 \mathrm{~min}$. at $4^{\circ} \mathrm{C}$. The cleared supernatant was purified by solid-phase extraction using Oasis HLB 1cc (30 mg) SPE columns (Waters). Columns were washed with 1ml methanol, equilibrated with $1 \mathrm{ml}$ water, loaded with sample, desalted with $1 \mathrm{ml}$ water, and eluted with $1 \mathrm{ml}$ methanol containing $25 \mathrm{mM}$ ammonium acetate. The purified extracts were evaporated to dryness under nitrogen and resuspended in $55 \mu \mathrm{l} 5 \%(\mathrm{w} / \mathrm{v}) 5$-sulfosalicylic acid (SSA) in optima HPLC grade water. Acetyl-CoA was measured by liquid chromatography-high resolution 
mass spectrometry. Briefly, $5 \mu$ l of sample in $5 \%$ SSA were analyzed by injection into an Ultimate 3000 HPLC coupled to a Q Exactive Plus (Thermo Scientific) mass spectrometer in positive ESI mode using the settings described previously (76). Calibration curves were prepared using analytical standards from Sigma Aldrich and processed identically as the samples. Data were integrated using Tracefinder v4.1 (Thermo Scientific) software, and additional statistical analysis conducted by Prism v7.05 (GraphPad). Acetyl-CoA values were normalized to cell number and reported as pmol/10 $10^{5}$ cells.

\section{Targeted metabolomics analysis of SIRT5 depletion}

Melanoma cell lines infected with a control (pLKO.1 empty vector), SIRT5 KD1 or KD2 virus were cultured for $72 \mathrm{hrs}$. in complete growth medium on $10 \mathrm{~cm}$ dishes in biological sextuplicate. Three dishes were reserved for metabolite collection, and three dishes were harvested in protein sample buffer $(62.5 \mathrm{mM}$ Tris $\mathrm{pH} 6.8,2 \%$ SDS, $10 \%$ glycerol) for protein quantification. A complete medium change was performed two hrs. prior to metabolite collection. Cells were washed twice in $2 \mathrm{ml}$ of PBS; one wash at room temperature and one at $4^{\circ} \mathrm{C}$. Taking care to aspirate all remaining liquid, plates were placed onto dry ice to cool before incubating in $4 \mathrm{ml}$ of $80 \%$ methanol for 10 mins. Plates were then scrape-harvested, and lysates transferred to $15 \mathrm{ml}$ conical tubes. Lysates were centrifuged at $200 \mathrm{xg}$ for 10 mins. For all experiments, the quantity of the metabolite fraction analyzed was adjusted to the corresponding average protein concentration. Liquid chromatography coupled-tandem mass spectrometry (LC-MS/MS) was employed for the detection of relative metabolite levels (116). Samples were analyzed on a 6490 Triple Quadrupole (QqQ) LC-MS against a targeted panel of 225 metabolites run in positive and negative modes. Agilent MassHunter Optimizer and Workstation Software LC-MS Data Acquisition for 6400 Series Triple Quadrupole B.08.00 was used for standard optimization and data acquisition. Agilent MassHunter Workstation Software Quantitative Analysis Version B.0700 for QqQ was used for initial raw data extraction and analysis. Each MRM transition and 
its retention time of left delta and right delta was $1 \mathrm{~min}$. Additional parameters include mass extraction window of 0.05 Da right and left from the extract m/z, Agile2 integrator algorithm, peak filter of 100 counts, noise algorithm RMS, noise SD multiplier of 5 min, S/N 3, Accuracy Max 20\% max \%Dev, and Quadratic/Cubic Savitzky-Golay smoothing algorithm with smoothing function width of 14 and Gaussian width of 5 . Peak area values under 10,000 were discarded as noise. Remaining raw values for each metabolite were median-centered across all conditions for each cell line. The average and standard deviation were taken for all replicates, and Student's ttests were conducted comparing each KD to the control. Data for each metabolite was represented as $\log (2)$ fold change with relative standard deviation. Pathway enrichment studies of both up- and down-regulated signaling were completed using Metaboanalyst and represented as $\log 10(p-v a l u e)(117)$

\section{CM-H2DCFDA staining for ROS measurement}

A2058 cells were plated at a density of $1 \times 10^{6}$ per well of two six-well plates. Three wells were transduced each with either a lentivirus expressing a non-silencing shRNA (control) or one of two shRNAs targeting SIRT5 (KD1 or KD2). Ninety-six hrs. after transduction, CM-H2DCFDA (ThermoFisher) was added to a final concentration of $5 \mu \mathrm{M}$ and incubated for 30 minutes in a humidified chamber at $37^{\circ} \mathrm{C}$. Cells were analyzed by flow cytometry using a BD FACSCalibur and results were plotted using FlowJo 10.2 analysis software.

\section{Co-immunoprecipitation of SIRT5 with MTHFD1L}

A2058 whole-cell protein extracts were prepared in protein lysis buffer $(50 \mathrm{mM}$ Tris $\mathrm{pH} 7.4$, $150 \mathrm{mM} \mathrm{NaCl}, 1 \%$ Triton X-100, 0.5\% NP40, 10\% Glycerol) supplemented with $1 \mu \mathrm{M}$ TSA, 10mM nicotinamide, PhosStop cocktail (Roche), and protease inhibitor cocktail (Roche). Lysates were sonicated for 30 seconds using a Branson Sonifier set to output "2." Lysates were then clarified by centrifugation at $15,000 \mathrm{rcf}$ for 30 minutes at $4^{\circ} \mathrm{C}$. Protein concentrations were 
determined using DC Protein Assay (Bio-Rad). Equivalent amounts (1mg) of total protein were mixed with the indicated amount (or $5 \mu \mathrm{g}$ ) of biotinylated anti-SIRT5 antibody or normal rabbit IgG, and $20 \mu$ l of protein A magnetic beads (Pierce), equilibrated in lysis buffer. After overnight rotation at $4^{\circ} \mathrm{C}$, samples were washed 4 times in $1 \mathrm{ml}$ of lysis buffer. Following the final wash, samples were boiled in $62.5 \mathrm{mM}$ Tris $\mathrm{pH} 6.8,2 \%$ SDS, and $10 \%$ glycerol, supplemented with 710mM $\beta$-mercaptoethanol and $0.01 \%(w / v)$ bromophenol blue for 5 mins, and fractionated by SDS-PAGE, followed by electrophoretic transfer and immunoblotting with the indicated antibodies.

Measurement of Oxygen consumption, Extracellular acidification, and Real-Time ATP production rate

Oxygen consumption rate (OCR), Extracellular acidification rate (ECAR), and Real-Time ATP production rate were measured using the XFe96 Extracellular Flux Analyzer (Seahorse Bioscience, Agilent Technologies, Santa Clara, CA). To measure glucose-dependent mitochondrial respiration, mitochondrial stress tests were performed to measure OCR per manufacturer's instructions. Briefly, 72 hrs. post-transduction, $4 \times 10^{4}$ A375 or A2058 cells were plated in DMEM complete media supplemented with $10 \%$ heat-inactivated FBS into each well of a 96-well Seahorse microplate. Cells were then incubated in $5 \% \mathrm{CO}_{2}$ at $37^{\circ} \mathrm{C}$ for $24 \mathrm{hrs}$. Following incubation, cells were washed twice, incubated in non- $\mathrm{CO}_{2}$ incubator at $37^{\circ} \mathrm{C}$, and analyzed in XF assay media (non-buffered DMEM containing 25mM glucose, and 1mM sodium pyruvate, $\mathrm{pH} 7.4$ ) at $37^{\circ} \mathrm{C}$, under basal conditions and in response to $2 \mu \mathrm{M}$ oligomycin (Sigma), $1 \mu \mathrm{M}$ fluoro-carbonyl cyanide phenylhydrazone (FCCP) (Sigma) and $0.5 \mu \mathrm{M}$ rotenone (Sigma)/0.5 $\mu \mathrm{M}$ antimycin A (Sigma). Data were analyzed by the Seahorse XF Cell Mito Stress Test Report Generator. OCR ( $\mathrm{pmol} \mathrm{O}_{2} / \mathrm{min}$ ) values were normalized to the protein content. 
To measure glutamine-dependent mitochondrial respiration, mitochondrial stress test was performed to measure OCR as per manufacturer's instructions. Briefly, 72 hrs. posttransduction, $4 \times 10^{4} \mathrm{~A} 375, \mathrm{~A} 2058$ or SK-MEL-2 cells were plated in DMEM complete media supplemented with $10 \%$ heat-inactivated FBS into each well of a 96 -well Seahorse microplate. Cells were then incubated in $5 \% \mathrm{CO}_{2}$ at $37^{\circ} \mathrm{C}$ for $24 \mathrm{hrs}$. Following incubation, cells were washed twice, incubated (in non- $\mathrm{CO}_{2}$ incubator at $37^{\circ} \mathrm{C}$ ), and analyzed in XF assay media (nonbuffered DMEM containing $4 \mathrm{mM} \mathrm{L-glutamine,} \mathrm{and} 1 \mathrm{mM}$ sodium pyruvate, $\mathrm{pH} 7.4$ ) at $37^{\circ} \mathrm{C}$, under basal conditions and in response to $2 \mu \mathrm{M}$ oligomycin (Sigma), $1 \mu \mathrm{M}$ fluoro-carbonyl cyanide phenylhydrazone (FCCP) (Sigma) and $0.5 \mu \mathrm{M}$ rotenone (Sigma)/0.5 $\mu \mathrm{M}$ antimycin $\mathrm{A}$ (Sigma). Data were analyzed by the Seahorse XF Cell Mito Stress Test Report Generator. OCR (pmol $\mathrm{O}_{2} / \mathrm{min}$ ) values were normalized to protein content.

ECAR values were measured by performing glycolysis stress tests according to manufacturer's instructions. Briefly, 72 hrs. post-transduction, $4 \times 10^{4}$ A375 and A2058 cells were plated in DMEM complete media supplemented with $10 \%$ heat-inactivated FBS into each well of a 96well Seahorse microplate. Cells were then incubated in $5 \% \mathrm{CO}_{2}$ at $37^{\circ} \mathrm{C}$ for $24 \mathrm{hrs}$. Following incubation, cells were washed twice, incubated in a non- $\mathrm{CO}_{2}$ incubator at $37^{\circ} \mathrm{C}$, and analyzed in XF assay media (non-buffered DMEM containing $2 \mathrm{mM} \mathrm{L-glutamine,} \mathrm{pH} 7.4$ ) at $37^{\circ} \mathrm{C}$, under basal conditions and in response to $10 \mathrm{mM}$ glucose (Sigma), $2 \mu \mathrm{M}$ oligomycin (Sigma), and 50mM 2-deoxy-D-glucose (Sigma). Data were analyzed by the Seahorse XF Cell Glycolysis Stress Test Report Generator. ECAR ( $\mathrm{mpH} / \mathrm{min}$ ) values were normalized to protein content.

The Seahorse XF Real-Time ATP Rate Assay Kit (Agilent) was used to simultaneously measure the basal ATP production rates from mitochondrial respiration and glycolysis. The assay was performed per manufacturer's instructions. Briefly, 72 hrs. post-transduction, $4 \times 10^{4}$ A375 and A2058 cells were plated in DMEM complete media supplemented with $10 \%$ heat-inactivated 
FBS into each well of a 96-well Seahorse microplate. Cells were then incubated in $5 \% \mathrm{CO}_{2}$ at $37^{\circ} \mathrm{C}$ for $24 \mathrm{hrs}$. Following incubation, cells were washed twice, incubated in a non- $\mathrm{CO}_{2}$ incubator at $37^{\circ} \mathrm{C}$, and analyzed in XF assay media (non-buffered DMEM containing $10 \mathrm{mM}$ glucose, $2 \mathrm{mM}$ L-glutamine, and $1 \mathrm{mM}$ sodium pyruvate, $\mathrm{pH} 7.4$ ) at $37^{\circ} \mathrm{C}$, under basal conditions and in response to $1.5 \mu \mathrm{M}$ oligomycin, and $0.5 \mu \mathrm{M}$ rotenone/0.5 $\mu \mathrm{M}$ antimycin $\mathrm{A}$. Data were analyzed by the Seahorse XF Real-Time ATP Rate Assay Report Generator. ATP production rates were normalized to protein content.

\section{JC-1 Mitochondrial membrane potential assay}

At 96 hrs. post-transduction approximately $2 \times 10^{5}$ A2058 cells in $100 \mu$ l warm PBS were transferred into tube and stained with $10 \mu \mathrm{g} / \mathrm{ml}$ of JC-1 dye (AnaSpec). An additional 100 $\mathrm{\mu l}$ from control cells were treated with $200 \mu \mathrm{M}$ FCCP, a mitochondrial uncoupler, which depolarizes mitochondrial membrane potential, and used as a positive control. After 30 minutes of staining, cells were centrifuged to remove excess JC-1, washed twice with warm PBS and resuspended in $100 \mu \mathrm{l}$ of warm PBS. Cells were transferred into black 96 -well plates and absorbance measured at 535nm (excitation)/595nm (emission) (aggregate, red) and 485nm (excitation)/435nm (emission) (monomer, green) using a H1 Synergy plate reader. The data are presented as a ratio of red to green fluorescence.

$\left[{ }^{13} C_{6}\right]$ Glucose, $\left[{ }^{13} C_{5}\right]$ Glutamine and $\left[{ }^{15} N_{2}\right]$ Glutamine Labelling for Metabolic Flux Glucose and glutamine labelling were carried out as described previously (58). Briefly, 72 hrs. after transduction with pLKO control, pLKO SIRT5 KD1, or pLKO SIRT5 KD2, cells were trypsinized and plated in triplicate in 6-well dishes at a density of $1 \times 10^{6}$ cells per well. Culture medium was replaced with $\left[{ }^{13} \mathrm{C}_{6}\right]$ glucose, $\left[{ }^{13} \mathrm{C}_{5}\right]$ glutamine or $\left[{ }^{15} \mathrm{~N}_{2}\right]$ glutamine labelling medium for 6 hours. Glucose labeling medium consisted of: MEM (1 g/L glucose; Gibco) supplemented with $1 \mathrm{~g} / \mathrm{L}\left[\mathrm{U}-{ }^{13} \mathrm{C}_{6}\right]$ glucose (Sigma), $10 \% \mathrm{v} / \mathrm{v}$ fetal bovine serum, $2 \mathrm{mM} \mathrm{L-glutamine,} 1 \% \mathrm{v} / \mathrm{v}$ 
pen/strep solution, and 1\% MEM non-essential amino acids. Glutamine labeling medium was prepared as the glucose labeling medium, except an additional $1 \mathrm{~g} / \mathrm{L}$ of unlabeled glucose, 1 $\mathrm{mM}$ unlabeled L-glutamine, and $1 \mathrm{mM}\left[\mathrm{U}-{ }^{13} \mathrm{C}_{5}\right] \mathrm{L}$-glutamine (Sigma) or $1 \mathrm{mM}\left[{ }^{15} \mathrm{~N}_{2}\right]$ L-glutamine (Sigma) was added. After 6 hours, cells were washed with cold PBS, then $0.45 \mathrm{ml}$ of $50 \%$ methanol:50\% water containing the internal standard, $20 \mu \mathrm{M} \mathrm{L-norvaline} \mathrm{(Sigma)} \mathrm{was} \mathrm{added} \mathrm{to}$ each well. Plates were frozen on dry ice for 30 minutes and thawed on ice for 10 minutes. The cell suspension was transferred to a microfuge tube followed by the addition of $0.225 \mathrm{ml}$ chloroform. Samples were vortexed, then centrifuged at $20,000 \mathrm{rcf}$ for 5 minutes at $4^{\circ} \mathrm{C}$. The top layer was transferred to a fresh tube and dried in a Speedvac. Samples were then shipped to the Sanford Burnham Prebys Medical Discovery Institute (La Jolla, CA) for GC/MS-based metabolic profiling. Derivatization of metabolites, GC/MS settings, and data analysis for stable isotope labeling and metabolite quantification were as described (61). Metabolite quantities were determined using mass ion peak areas corresponding to unlabeled metabolites, and then corrected for the fraction of a metabolite that was ${ }^{13} \mathrm{C}$ or ${ }^{15} \mathrm{~N}$ labeled to yield the total (labeled plus unlabeled) cellular quantity for that metabolite. This amount was normalized to total cellular protein.

\section{Statistical Analysis}

All statistical analyses were performed using Prism graphing software (Graphpad). Unless otherwise noted, $\mathrm{p} \leq 0.05$ produced from an unpaired Student's t-test was considered significant.

\section{Study approval}

All mice were housed at the Biomedical Science Research Building (UM). All vertebrate animal experiments were approved by and performed in accordance with the regulations of the University Committee on Use and Care of Animals. 


\section{Author Contributions:}

WG, LBR, AHG, SK, ACM, AMM, MES, MA, ASAM, CHC, NK, KAM, HJL, LZ, PS, ST, ELV, SI, MW, JSW, HPS, RAS, ALP, AA, RAS, MSS, DAS, DRF, MWB, SC, ZNC, MEV, NWS, MHR, ALO, CAL performed experiments and/or analyzed data. WG and DBL interpreted data, wrote and revised the paper. WG made the figures. DBL supervised overall design and study interpretation. 
Acknowledgements: Funding: Melanoma Research Alliance (DL and CAL), NIH R01GM101171, AACR-Bayer (17-80-44-LOMB), DoD awards CA170628, CA190267, OC140123, and NF170044 (DL), and the Rogel Cancer Center. Use of the Rogel Cancer Center shared resources is also gratefully acknowledged by DL and CAL (P30CA46592). SK was supported in part through an award from the Pablove Foundation. CAL was supported by a 2017 AACR NextGen Grant for Transformative Cancer Research (17-20-01-LYSS), an ACS Research Scholar Grant (RSG-18-186-01), and NIH award R01CA244931. ZNC was supported by NIH R01CA217141 and AACR-Bayer (19-80-44-NIKO). Metabolomics studies performed at the University of Michigan were supported by NIH grant DK097153. ALP is supported by the Highlands and Islands Enterprise (HMS9353763); MSS holds a fellowship (APP1106491) from the National Health and Medical Research Council (NHMRC). WG, AG, AM and LR were supported by NIH T32 awards (WG: GM007544, AG000114, HL007853 and AR007917; AG: AG000114 and GM113900; AM: AG000114; LR NL007517). NWS was supported by R01GM132261. ST was funded by American Diabetes Association postdoctoral fellowship \#118-PDF-144. RAS and JSW are supported by the Australian National Health and Medical Research Council Fellowship program. RAS is also supported by a National Health and Medical Research Council of Australia Program Grant (APP1093017). Support from colleagues at Melanoma Institute Australia and the Royal Prince Alfred Hospital is also gratefully acknowledged. HPS holds an NHMRC MRFF Next Generation Clinical Researchers Program Practitioner Fellowship (APP1137127). The SBP Cancer Metabolism Core is supported by National Cancer Institute Cancer Center Support Grant P30 030199. Dr. Jeongsoon Park is acknowledged for technical contributions early in the project. Dr. Scott Pletcher (UM) is acknowledged for assistance with statistical analysis. Drs. Emily Bernstein (Icahn School of Medicine at Mount Sinai) and Kathleen Cho (UM) are acknowledged for generously providing cell lines. Drs. David Fisher (MGH Cancer Center/Harvard Medical School) and Kathryn Wellen (University of Pennsylvania) are gratefully acknowledged for helpful discussions. Olga Zagnitko 
bioRxiv preprint doi: https://doi.org/10.1101/2020.09.07.286526; this version posted February 24, 2021. The copyright holder for this preprint (which was not certified by peer review) is the author/funder. All rights reserved. No reuse allowed without permission.

is acknowledged for assistance with GC/MS measurements. Drs. Robert Weiss, Hening Lin, and Michael Deininger are acknowledged for discussion of unpublished data. GEO submission of RNA-seq data is in process. 


\section{References}

1. Siegel RL, Miller KD, Fuchs HE, and Jemal A. Cancer Statistics, 2021. CA Cancer J Clin. 2021;71(1):7-33.

2. Siegel RL, Miller KD, and Jemal A. Cancer statistics, 2019. CA: A Cancer Journal for Clinicians. 2019;69(1):7-34.

3. Dharmadhikari N, Mehnert JM, and Kaufman HL. Oncolytic virus immunotherapy for melanoma. Current treatment options in oncology. 2015;16(3):326.

4. Guy GP, and Ekwueme DU. Years of potential life lost and indirect costs of melanoma and non-melanoma skin cancer: a systematic review of the literature.

PharmacoEconomics. 2011;29(10):863-74.

5. Larkin J, Chiarion-Sileni V, Gonzalez R, Grob JJ, Rutkowski P, Lao CD, et al. Five-Year Survival with Combined Nivolumab and Ipilimumab in Advanced Melanoma. $N$ Engl $J$ Med. 2019;381(16):1535-46.

6. Singh M, Durairaj P, and Yeung J. Uveal Melanoma: A Review of the Literature. Oncology and Therapy. 2018;6(1):87-104.

7. Bringman-Rodenbarger LR, Guo AH, Lyssiotis CA, and Lombard DB. Emerging Roles for SIRT5 in Metabolism and Cancer. Antioxid Redox Signal. 2018;28(8):677-90.

8. Du J, Zhou Y, Su X, Yu JJ, Khan S, Jiang H, et al. Sirt5 is a NAD-dependent protein lysine demalonylase and desuccinylase. Science. 2011;334(6057):806-9.

9. Rardin MJ, He W, Nishida Y, Newman JC, Carrico C, Danielson SR, et al. SIRT5 regulates the mitochondrial lysine succinylome and metabolic networks. Cell metabolism. 2013;18(6):920-33.

10. North BJ, Marshall BL, Borra MT, Denu JM, and Verdin E. The human Sir2 ortholog, SIRT2, is an NAD+-dependent tubulin deacetylase. Mol Cell. 2003;11(2):437-44.

11. Park J, Chen Y, Tishkoff DX, Peng C, Tan M, Dai L, et al. SIRT5-mediated lysine desuccinylation impacts diverse metabolic pathways. Mol Cell. 2013;50(6):919-30. 
12. Tan M, Peng C, Anderson Kristin A, Chhoy P, Xie Z, Dai L, et al. Lysine Glutarylation Is a Protein Posttranslational Modification Regulated by SIRT5. Cell metabolism. 2014;19(4):605-17.

13. Peng C, Lu Z, Xie Z, Cheng Z, Chen Y, Tan M, et al. The first identification of lysine malonylation substrates and its regulatory enzyme. Molecular \& cellular proteomics : MCP. 2011;10(12):M111 012658 1-12.

14. Nishida Y, Rardin MJ, Carrico C, He W, Sahu AK, Gut P, et al. SIRT5 Regulates both Cytosolic and Mitochondrial Protein Malonylation with Glycolysis as a Major Target. Mol Cell. 2015;59(2):321-32.

15. Lombard DB, Alt FW, Cheng HL, Bunkenborg J, Streeper RS, Mostoslavsky R, et al. Mammalian Sir2 homolog SIRT3 regulates global mitochondrial lysine acetylation. Molecular and cellular biology. 2007;27(24):8807-14.

16. Yu J, Sadhukhan S, Noriega LG, Moullan N, He B, Weiss RS, et al. Metabolic characterization of a Sirt5 deficient mouse model. Scientific reports. 2013;3:2806.

17. Kumar S, and Lombard DB. Functions of the sirtuin deacylase SIRT5 in normal physiology and pathobiology. Crit Rev Biochem Mol Biol. 2018;53(3):311-34.

18. Boylston JA, Sun J, Chen Y, Gucek M, Sack MN, and Murphy E. Characterization of the cardiac succinylome and its role in ischemia-reperfusion injury. $\mathrm{J} \mathrm{Mol} \mathrm{Cell} \mathrm{Cardiol.}$ 2015;88:73-81.

19. Sadhukhan S, Liu X, Ryu D, Nelson OD, Stupinski JA, Li Z, et al. Metabolomics-assisted proteomics identifies succinylation and SIRT5 as important regulators of cardiac function. Proc Natl Acad Sci U S A. 2016;113(16):4320-5.

20. Hershberger KA, Abraham DM, Martin AS, Mao L, Liu J, Gu H, et al. Sirtuin 5 is required for mouse survival in response to cardiac pressure overload. J Biol Chem. 2017;292(48):19767-81. 
21. Hershberger KA, Abraham DM, Liu J, Locasale JW, Grimsrud PA, and Hirschey MD. Ablation of Sirtuin5 in the postnatal mouse heart results in protein succinylation and normal survival in response to chronic pressure overload. J Biol Chem. 2018;293(27):10630-45.

22. Roth M, and Chen WY. Sorting out functions of sirtuins in cancer. Oncogene. 2014;33(13):1609-20.

23. Ohanna M, Bonet C, Bille K, Allegra M, Davidson I, Bahadoran P, et al. SIRT1 promotes proliferation and inhibits the senescence-like phenotype in human melanoma cells. Oncotarget. 2014;5(8):2085-95.

24. Bajpe PK, Prahallad A, Horlings H, Nagtegaal I, Beijersbergen R, and Bernards R. A chromatin modifier genetic screen identifies SIRT2 as a modulator of response to targeted therapies through the regulation of MEK kinase activity. Oncogene. 2015;34(4):531-6.

25. George J, Nihal M, Singh CK, Zhong W, Liu X, and Ahmad N. Pro-Proliferative Function of Mitochondrial Sirtuin Deacetylase SIRT3 in Human Melanoma. J Invest Dermatol. 2016;136(4):809-18.

26. Garcia-Peterson LM, Ndiaye MA, Singh CK, Chhabra G, Huang W, and Ahmad N. SIRT6 histone deacetylase functions as a potential oncogene in human melanoma. Genes Cancer. 2017;8(9-10):701-12.

27. Strub T, Ghiraldini FG, Carcamo S, Li M, Wroblewska A, Singh R, et al. SIRT6 haploinsufficiency induces BRAF(V600E) melanoma cell resistance to MAPK inhibitors via IGF signalling. Nat Commun. 2018;9(1):3440.

28. Lu W, Zuo Y, Feng Y, and Zhang M. SIRT5 facilitates cancer cell growth and drug resistance in non-small cell lung cancer. Tumour biology : the journal of the International Society for Oncodevelopmental Biology and Medicine. 2014. 
29. Lv XB, Liu L, Cheng C, Yu B, Xiong L, Hu K, et al. SUN2 exerts tumor suppressor functions by suppressing the Warburg effect in lung cancer. Scientific reports. 2015;5:17940.

30. Lin ZF, Xu HB, Wang JY, Lin Q, Ruan Z, Liu FB, et al. SIRT5 desuccinylates and activates SOD1 to eliminate ROS. Biochemical and biophysical research communications. 2013;441(1):191-5.

31. Zhou L, Wang F, Sun R, Chen X, Zhang M, Xu Q, et al. SIRT5 promotes IDH2 desuccinylation and G6PD deglutarylation to enhance cellular antioxidant defense. EMBO Rep. 2016;17(6):811-22.

32. Yang X, Wang Z, Li X, Liu B, Liu M, Liu L, et al. SHMT2 Desuccinylation by SIRT5 Drives Cancer Cell Proliferation. Cancer Res. 2018;78(2):372-86.

33. Chang L, Xi L, Liu Y, Liu R, Wu Z, and Jian Z. SIRT5 promotes cell proliferation and invasion in hepatocellular carcinoma by targeting E2F1. Mol Med Rep. 2017.

34. Zhang R, Wang C, Tian Y, Yao Y, Mao J, Wang H, et al. SIRT5 Promotes Hepatocellular Carcinoma Progression by Regulating Mitochondrial Apoptosis. J Cancer. 2019;10(16):3871-82.

35. Greene KS, Lukey MJ, Wang X, Blank B, Druso JE, Lin MJ, et al. SIRT5 stabilizes mitochondrial glutaminase and supports breast cancer tumorigenesis. Proc Natl Acad Sci U S A. 2019.

36. Pavlova NN, and Thompson CB. The Emerging Hallmarks of Cancer Metabolism. Cell metabolism. 2016;23(1):27-47.

37. Abril YLN, Fernandez IR, Hong JY, Chiang YL, Kutateladze DA, Zhao Q, et al. Pharmacological and genetic perturbation establish SIRT5 as a promising target in breast cancer. Oncogene. 2021. 
38. Li F, He X, Ye D, Lin Y, Yu H, Yao C, et al. NADP(+)-IDH Mutations Promote Hypersuccinylation that Impairs Mitochondria Respiration and Induces Apoptosis Resistance. Mol Cell. 2015;60(4):661-75.

39. Xu YS, Liang JJ, Wang Y, Zhao XJ, Xu L, Xu YY, et al. STAT3 Undergoes Acetylationdependent Mitochondrial Translocation to Regulate Pyruvate Metabolism. Scientific reports. 2016;6:39517.

40. Hartman ML, and Czyz M. Pro-Survival Role of MITF in Melanoma. Journal of Investigative Dermatology. 2015;135(2):352-8.

41. Zhuang D, Mannava S, Grachtchouk V, Tang WH, Patil S, Wawrzyniak JA, et al. C-MYC overexpression is required for continuous suppression of oncogene-induced senescence in melanoma cells. Oncogene. 2008;27(52):6623-34.

42. Singleton KR, Crawford L, Tsui E, Manchester HE, Maertens O, Liu X, et al. Melanoma Therapeutic Strategies that Select against Resistance by Exploiting MYC-Driven Evolutionary Convergence. Cell Rep. 2017;21(10):2796-812.

43. North JP, Vetto JT, Murali R, White KP, White CR, Jr., and Bastian BC. Assessment of copy number status of chromosomes 6 and 11 by FISH provides independent prognostic information in primary melanoma. The American journal of surgical pathology. $2011 ; 35(8): 1146-50$.

44. Santos GC, Zielenska M, Prasad M, and Squire JA. Chromosome $6 p$ amplification and cancer progression. J Clin Pathol. 2007;60(1):1-7.

45. Cancer Genome Atlas N. Genomic Classification of Cutaneous Melanoma. Cell. 2015;161(7):1681-96.

46. Hodis E, Watson IR, Kryukov GV, Arold ST, Imielinski M, Theurillat JP, et al. A landscape of driver mutations in melanoma. Cell. 2012;150(2):251-63.

47. Wu D, Li Y, Zhu KS, Wang H, and Zhu WG. Advances in Cellular Characterization of the Sirtuin Isoform, SIRT7. Front Endocrinol (Lausanne). 2018;9:652. 
48. Stark M, and Hayward N. Genome-wide loss of heterozygosity and copy number analysis in melanoma using high-density single-nucleotide polymorphism arrays. Cancer Res. 2007;67(6):2632-42.

49. Dutton-Regester K, Aoude LG, Nancarrow DJ, Stark MS, O'Connor L, Lanagan C, et al. Identification of TFG (TRK-fused gene) as a putative metastatic melanoma tumor suppressor gene. Genes Chromosomes Cancer. 2012;51(5):452-61.

50. Stark MS, Tan JM, Tom L, Jagirdar K, Lambie D, Schaider H, et al. Whole-Exome Sequencing of Acquired Nevi Identifies Mechanisms for Development and Maintenance of Benign Neoplasms. J Invest Dermatol. 2018;138(7):1636-44.

51. Shain AH, Yeh I, Kovalyshyn I, Sriharan A, Talevich E, Gagnon A, et al. The Genetic Evolution of Melanoma from Precursor Lesions. New England Journal of Medicine. 2015;373(20):1926-36.

52. Luebker SA, and Koepsell SA. Diverse Mechanisms of BRAF Inhibitor Resistance in Melanoma Identified in Clinical and Preclinical Studies. Front Oncol. 2019;9:268.

53. Meeth K, Wang JX, Micevic G, Damsky W, and Bosenberg MW. The YUMM lines: a series of congenic mouse melanoma cell lines with defined genetic alterations. Pigment Cell \& Melanoma Research. 2016;29(5):590-7.

54. Hartman ML. Non-Apoptotic Cell Death Signaling Pathways in Melanoma. Int J Mol Sci. $2020 ; 21(8)$.

55. Dankort D, Curley DP, Cartlidge RA, Nelson B, Karnezis AN, Damsky WE, Jr., et al. Braf(V600E) cooperates with Pten loss to induce metastatic melanoma. Nat Genet. 2009;41(5):544-52.

56. Buler M, Aatsinki SM, Izzi V, Uusimaa J, and Hakkola J. SIRT5 is under the control of PGC-1alpha and AMPK and is involved in regulation of mitochondrial energy metabolism. FASEB journal : official publication of the Federation of American Societies for Experimental Biology. 2014;28(7):3225-37. 
57. Zhang Y, Bharathi SS, Rardin MJ, Lu J, Maringer KV, Sims-Lucas S, et al. Lysine desuccinylase SIRT5 binds to cardiolipin and regulates the electron transport chain. $J$ Biol Chem. 2017;292(24):10239-49.

58. Scott DA, Richardson AD, Filipp FV, Knutzen CA, Chiang GG, Ronai ZA, et al. Comparative metabolic flux profiling of melanoma cell lines: beyond the Warburg effect. J Biol Chem. 2011;286(49):42626-34.

59. Filipp FV, Scott DA, Ronai ZA, Osterman AL, and Smith JW. Reverse TCA cycle flux through isocitrate dehydrogenases 1 and 2 is required for lipogenesis in hypoxic melanoma cells. Pigment Cell Melanoma Res. 2012;25(3):375-83.

60. Filipp FV, Ratnikov B, De Ingeniis J, Smith JW, Osterman AL, and Scott DA. Glutaminefueled mitochondrial metabolism is decoupled from glycolysis in melanoma. Pigment Cell Melanoma Res. 2012;25(6):732-9.

61. Ratnikov B, Aza-Blanc P, Ronai ZA, Smith JW, Osterman AL, and Scott DA. Glutamate and asparagine cataplerosis underlie glutamine addiction in melanoma. Oncotarget. 2015;6(10):7379-89.

62. Qin JZ, Xin H, and Nickoloff BJ. Targeting glutamine metabolism sensitizes melanoma cells to TRAIL-induced death. Biochemical and biophysical research communications. 2010;398(1):146-52.

63. Qin JZ, Xin H, and Nickoloff BJ. 2-deoxyglucose sensitizes melanoma cells to TRAILinduced apoptosis which is reduced by mannose. Biochemical and biophysical research communications. 2010;401(2):293-9.

64. Garraway LA, Widlund HR, Rubin MA, Getz G, Berger AJ, Ramaswamy S, et al. Integrative genomic analyses identify MITF as a lineage survival oncogene amplified in malignant melanoma. Nature. 2005;436(7047):117-22.

65. Hoek KS, and Goding CR. Cancer stem cells versus phenotype-switching in melanoma. Pigment Cell \& Melanoma Research. 2010;23(6):746-59. 
66. Müller J, Krijgsman O, Tsoi J, Robert L, Hugo W, Song C, et al. Low MITF/AXL ratio predicts early resistance to multiple targeted drugs in melanoma. Nature Communications. 2014;5(1):5712.

67. Cheli Y, Guiliano S, Botton T, Rocchi S, Hofman V, Hofman P, et al. Mitf is the key molecular switch between mouse or human melanoma initiating cells and their differentiated progeny. Oncogene. 2011;30(20):2307-18.

68. Seoane M, Buhs S, Iglesias P, Strauss J, Puller AC, Muller J, et al. Lineage-specific control of TFIIH by MITF determines transcriptional homeostasis and DNA repair. Oncogene. 2019;38(19):3616-35.

69. Duarte NC, Becker SA, Jamshidi N, Thiele I, Mo ML, Vo TD, et al. Global reconstruction of the human metabolic network based on genomic and bibliomic data. Proc Natl Acad Sci U S A. 2007;104(6):1777-82.

70. Chandrasekaran S, Zhang J, Sun Z, Zhang L, Ross CA, Huang YC, et al. Comprehensive Mapping of Pluripotent Stem Cell Metabolism Using Dynamic GenomeScale Network Modeling. Cell Rep. 2017;21(10):2965-77.

71. Shlomi T, Cabili MN, Herrgard MJ, Palsson BO, and Ruppin E. Network-based prediction of human tissue-specific metabolism. Nat Biotechnol. 2008;26(9):1003-10.

72. Wellen KE, Hatzivassiliou G, Sachdeva UM, Bui TV, Cross JR, and Thompson CB. ATPcitrate lyase links cellular metabolism to histone acetylation. Science. 2009;324(5930):1076-80.

73. Gates LA, Shi J, Rohira AD, Feng Q, Zhu B, Bedford MT, et al. Acetylation on histone $\mathrm{H} 3$ lysine 9 mediates a switch from transcription initiation to elongation. The Journal of biological chemistry. 2017;292(35):14456-72.

74. Sivanand S, Viney I, and Wellen KE. Spatiotemporal Control of Acetyl-CoA Metabolism in Chromatin Regulation. Trends Biochem Sci. 2018;43(1):61-74. 
75. Snyder NW, Tombline G, Worth AJ, Parry RC, Silvers JA, Gillespie KP, et al. Production of stable isotope-labeled acyl-coenzyme A thioesters by yeast stable isotope labeling by essential nutrients in cell culture. Anal Biochem. 2015;474:59-65.

76. Frey AJ, Feldman DR, Trefely S, Worth AJ, Basu SS, and Snyder NW. LCquadrupole/Orbitrap high-resolution mass spectrometry enables stable isotope-resolved simultaneous quantification and (1)(3)C-isotopic labeling of acyl-coenzyme A thioesters. Anal Bioanal Chem. 2016;408(13):3651-8.

77. Yang $\mathrm{M}$, and Vousden $\mathrm{KH}$. Serine and one-carbon metabolism in cancer. Nat Rev Cancer. 2016;16(10):650-62.

78. Gao X, Reid MA, Kong M, and Locasale JW. Metabolic interactions with cancer epigenetics. Molecular Aspects of Medicine. 2017;54:50-7.

79. Mailloux RJ, McBride SL, and Harper ME. Unearthing the secrets of mitochondrial ROS and glutathione in bioenergetics. Trends Biochem Sci. 2013;38(12):592-602.

80. Colak G, Pougovkina O, Dai L, Tan M, Te Brinke H, Huang H, et al. Proteomic and Biochemical Studies of Lysine Malonylation Suggest Its Malonic Aciduria-associated Regulatory Role in Mitochondrial Function and Fatty Acid Oxidation. Molecular \& cellular proteomics : MCP. 2015;14(11):3056-71.

81. Kumar S, and Lombard DB. Mitochondrial sirtuins and their relationships with metabolic disease and cancer. Antioxid Redox Signal. 2015;22(12):1060-77.

82. Hartman ML, and Czyz M. MITF in melanoma: mechanisms behind its expression and activity. Cellular and Molecular Life Sciences. 2015;72(7):1249-60.

83. Kim E, Zucconi BE, Wu M, Nocco SE, Meyers DJ, McGee JS, et al. MITF Expression Predicts Therapeutic Vulnerability to p300 Inhibition in Human Melanoma. Cancer Res. 2019;79(10):2649-61.

84. Moon H, Zhu J, and White AC. Sirt5 is dispensable for Braf(V600E) -mediated cutaneous melanoma development and growth in vivo. Exp Dermatol. 2019;28(1):83-5. 
85. Haq R, and Fisher DE. Biology and clinical relevance of the micropthalmia family of transcription factors in human cancer. J Clin Oncol. 2011;29(25):3474-82.

86. Wellbrock C, and Marais R. Elevated expression of MITF counteracts B-RAF-stimulated melanocyte and melanoma cell proliferation. J Cell Biol. 2005;170(5):703-8.

87. Becker JS, Nicetto D, and Zaret KS. H3K9me3-Dependent Heterochromatin: Barrier to Cell Fate Changes. Trends Genet. 2016;32(1):29-41.

88. Li X, Egervari G, Wang Y, Berger SL, and Lu Z. Regulation of chromatin and gene expression by metabolic enzymes and metabolites. Nat Rev Mol Cell Biol. 2018;19(9):563-78.

89. Latham T, Mackay L, Sproul D, Karim M, Culley J, Harrison DJ, et al. Lactate, a product of glycolytic metabolism, inhibits histone deacetylase activity and promotes changes in gene expression. Nucleic Acids Res. 2012;40(11):4794-803.

90. Schlicker C, Gertz M, Papatheodorou P, Kachholz B, Becker CFW, and Steegborn C. Substrates and Regulation Mechanisms for the Human Mitochondrial Sirtuins Sirt3 and Sirt5. Journal of Molecular Biology. 2008;382(3):790-801.

91. Roessler C, Nowak T, Pannek M, Gertz M, Nguyen GT, Scharfe M, et al. Chemical probing of the human sirtuin 5 active site reveals its substrate acyl specificity and peptide-based inhibitors. Angew Chem Int Ed Engl. 2014;53(40):10728-32.

92. Polletta L, Vernucci E, Carnevale I, Arcangeli T, Rotili D, Palmerio S, et al. SIRT5 regulation of ammonia-induced autophagy and mitophagy. Autophagy. 2015;11(2):25370.

93. Guetschow ED, Kumar S, Lombard DB, and Kennedy RT. Identification of sirtuin 5 inhibitors by ultrafast microchip electrophoresis using nanoliter volume samples. Anal Bioanal Chem. 2016;408(3):721-31. 
94. Yang L-L, He Y-Y, Chen Q-L, Qian S, and Wang Z-Y. Design and Synthesis of New 9Substituted Norharmane Derivatives as Potential Sirt5 Inhibitors. Journal of Heterocyclic Chemistry. 2017;54(2):1457-66.

95. Sanson KR, Hanna RE, Hegde M, Donovan KF, Strand C, Sullender ME, et al. Optimized libraries for CRISPR-Cas9 genetic screens with multiple modalities. Nat Commun. 2018;9(1):5416.

96. Birsoy K, Wang T, Chen WW, Freinkman E, Abu-Remaileh M, and Sabatini DM. An Essential Role of the Mitochondrial Electron Transport Chain in Cell Proliferation Is to Enable Aspartate Synthesis. Cell. 2015;162(3):540-51.

97. Ran FA, Hsu PD, Wright J, Agarwala V, Scott DA, and Zhang F. Genome engineering using the CRISPR-Cas9 system. Nat Protoc. 2013;8(11):2281-308.

98. Sanjana NE, Shalem O, and Zhang F. Improved vectors and genome-wide libraries for CRISPR screening. Nat Methods. 2014;11(8):783-4.

99. Bousquet PF, Brana MF, Conlon D, Fitzgerald KM, Perron D, Cocchiaro C, et al. Preclinical evaluation of LU 79553: a novel bis-naphthalimide with potent antitumor activity. Cancer Res. 1995;55(5):1176-80.

100. Faustino-Rocha A, Oliveira PA, Pinho-Oliveira J, Teixeira-Guedes C, Soares-Maia R, da Costa RG, et al. Estimation of rat mammary tumor volume using caliper and ultrasonography measurements. Lab Anim (NY). 2013;42(6):217-24.

101. Andrews S. FastQC: a quality control tool for high throughput sequence data. Available online at: http://wwwbioinformaticsbabrahamacuk/projects/fastqc. 2010.

102. Ewels P, Magnusson M, Lundin S, and Kaller M. MultiQC: summarize analysis results for multiple tools and samples in a single report. Bioinformatics. 2016;32(19):3047-8.

103. Bolger AM, Lohse M, and Usadel B. Trimmomatic: a flexible trimmer for Illumina sequence data. Bioinformatics. 2014;30(15):2114-20. 
104. Dobin A, Davis CA, Schlesinger F, Drenkow J, Zaleski C, Jha S, et al. STAR: ultrafast universal RNA-seq aligner. Bioinformatics. 2013;29(1):15-21.

105. Liao Y, Smyth GK, and Shi W. featureCounts: an efficient general purpose program for assigning sequence reads to genomic features. Bioinformatics. 2014;30(7):923-30.

106. Love MI, Huber W, and Anders S. Moderated estimation of fold change and dispersion for RNA-seq data with DESeq2. Genome Biol. 2014;15(12):550.

107. Zhu A, Ibrahim JG, and Love MI. Heavy-tailed prior distributions for sequence count data: removing the noise and preserving large differences. Bioinformatics. 2019;35(12):2084-92.

108. Cancer Genome Atlas Research N, Weinstein JN, Collisson EA, Mills GB, Shaw KR, Ozenberger BA, et al. The Cancer Genome Atlas Pan-Cancer analysis project. Nat Genet. 2013;45(10):1113-20.

109. Liu Y, Sun J, and Zhao M. ONGene: A literature-based database for human oncogenes. J Genet Genomics. 2017;44(2):119-21.

110. Tsoi J, Robert L, Paraiso K, Galvan C, Sheu KM, Lay J, et al. Multi-stage Differentiation Defines Melanoma Subtypes with Differential Vulnerability to Drug-Induced IronDependent Oxidative Stress. Cancer Cell. 2018;33(5):890-904 e5.

111. Subramanian A, Tamayo P, Mootha VK, Mukherjee S, Ebert BL, Gillette MA, et al. Gene set enrichment analysis: a knowledge-based approach for interpreting genome-wide expression profiles. Proc Natl Acad Sci U S A. 2005;102(43):15545-50.

112. Liberzon A, Subramanian A, Pinchback R, Thorvaldsdottir H, Tamayo P, and Mesirov JP. Molecular signatures database (MSigDB) 3.0. Bioinformatics. 2011;27(12):1739-40.

113. Sengupta D, Kannan A, Kern M, Moreno MA, Vural E, Stack B, Jr., et al. Disruption of BRD4 at H3K27Ac-enriched enhancer region correlates with decreased c-Myc expression in Merkel cell carcinoma. Epigenetics. 2015;10(6):460-6. 
114. Shen F, Boccuto L, Pauly R, Srikanth S, and Chandrasekaran S. Genome-scale network model of metabolism and histone acetylation reveals metabolic dependencies of histone deacetylase inhibitors. Genome Biol. 2019;20(1):49.

115. Shen F, Cheek C, and Chandrasekaran S. Dynamic Network Modeling of Stem Cell Metabolism. Methods Mol Biol. 2019;1975:305-20.

116. Lee HJ, Kremer DM, Sajjakulnukit $P$, Zhang L, and Lyssiotis CA. A large-scale analysis of targeted metabolomics data from heterogeneous biological samples provides insights into metabolite dynamics. Metabolomics. 2019;15(7):103.

117. Chong J, Yamamoto M, and Xia J. MetaboAnalystR 2.0: From Raw Spectra to Biological Insights. Metabolites. 2019;9(3). 


\section{Legends}

Figure 1 Increased SIRT5 copy number in human melanoma.

A. Gain of extra SIRT5 copies in melanoma. BRAF, NRAS, PTEN, MITF, NF1 and other sirtuins are shown for comparison ( $\mathrm{n}=287$; data from TCGA, Provisional, analyzed on cBioPortal). ND, not determined. Percentage of samples with any genomic alteration (Any) or amplification or gain (Amp/Gain) is indicated. Graphed are any alterations queried for the indicated gene. Copy number gain indicates a low-level gain of a single additional copy, and amplification refers to high-level amplification (multiple extra copies). Results from the query (GENE: MUT AMP HOMDEL GAIN HETLOSS) in cBioPortal were analyzed and plotted. B. Kaplan-Meier analysis of overall survival in melanoma patients with or without copy number gain or amplification of SIRT5. Overall survival was analyzed using the query: "SIRT5: AMP GAIN." C. SIRT5 (6p23) and centromere $6 \mathrm{p}$ (Cen6p) amplification (amp) or co-amplification (Co-amp) in melanoma, as assayed by FISH staining ( $n=32)$. D. Sirtuin gene copy number $(C N)$ in human melanoma samples, as assayed by high density SNP array ( $n=139)$. E. SIRT5 mRNA expression levels in melanoma correlate with Clark's level ( $p=0.0044$, linear regression; $p=0.037$, ANOVA). F. SIRT5 protein levels are increased in melanoma relative to benign melanocytic lesions $(p=0.0333$, Chisquared; n=14 nevi, n=87 melanoma). See also Figure S1 and Table S1.

Figure 2 SIRT5 is required for melanoma cell growth and survival.

A. BRAF or NRAS mutant melanoma cell lines indicated were infected with a non-targeting shRNA (control) or one of two SIRT5 shRNAs (KD1 or KD2). Equivalent cell numbers were then plated $48 \mathrm{hrs}$. post-transduction into 96-well plates in the presence of puromycin. Cell mass was determined at the indicated timepoints via WST-1 assay, with absorbance measured at 450nm. Average results ( $n=6 /$ timepoint) are graphed. Error bars represent standard deviation. Representative of 5/5 SIRT5 shRNAs tested (see also Figure 3B). B. SIRT5 KD results in significantly ( $p<0.0001$, unpaired Student's t-test) impaired colony formation by A2058 and SK- 
MEL-2 cells 12 days post-transduction. Cell mass was assayed using crystal violet staining, with absorbance measured at 590nm. Average of $n=12$ technical replicates results are plotted. Error bars represent SD. Representative crystal violet-stained wells are shown. Lower panel, representative immunoblot analysis demonstrating SIRT5 KD. C. Top panel, viability of A2058 cells transfected with the indicated CRISPR guide RNA (Control or G1-G4). Cell mass was assayed using crystal violet staining, with absorbance measured at $590 \mathrm{~nm}$. Average of $n=9$ technical replicates results are plotted. Error bars represent standard deviation. Significance calculated using unpaired Student's t-test. Bottom panel, representative immunoblot analysis confirming CRISPR-mediated SIRT5 loss (Control: empty vector).

\section{Figure 3 SIRT5 depletion rapidly induces apoptosis in melanoma cells.}

A. Immunoblot analysis demonstrating induction of caspase 3 cleavage 72 and 96 hrs. posttransduction with shRNAs targeting SIRT5 (KD1 or KD2) in A2058 and SK-MEL-2 cell lines. B. Viability of MP-41, A2058 or YUMM5.2 cells infected with control (C) or one of five SIRT5 shRNAs (KD1-KD5) against human SIRT5 (top and middle panels) or mouse Sirt5 (bottom panel). Average results ( $n=6 /$ timepoint) are graphed. Error bars represent standard deviation. Right panels: immunoblot analysis demonstrating loss of SIRT5 and induction of caspase 3 cleavage following SIRT5 KD. C. Flow cytometric analysis of A2058 cells stained with Annexin $\mathrm{V}$ and propidium iodide (PI), as indicated, showing an increased fraction of Annexin V-positive cells 96 hrs. after SIRT5 KD. D. Average of $n=3$ technical replicates is plotted. Error bars represent (SD). Significance calculated using unpaired Student's t-test. Increased Annexin V ${ }^{+}$ staining is observed in both the PI-positive and PI-negative populations.

\section{Figure 4 SIRT5 loss-of-function inhibits melanoma tumor growth in vivo.}

A. SIRT5 depletion in A2058 cells results in attenuated xenograft tumor growth. Quantification of tumor size was initiated on day 13 after initial injection of cells (left panel). Tumor size was 
recorded with Vernier calipers on the days indicated. Each point represents the measurements on $n=5$ mice for each condition (C, KD1, or KD2). Pairwise representation of endpoint tumor size in each mouse within each group is plotted (right panel). Average tumor mass measurements at day 28 are plotted $(p<0.05$, paired two-tailed t-test for each group). Error bars represent SD. B. Mice were sacrificed, and tumors were dissected at 28 days after initial injection. Scale bar below tumors $=2 \mathrm{~cm}$. C. SIRT5 deficiency attenuates tumor formation in an autochthonous melanoma model. Sirt5 deficient-mice were bred into the

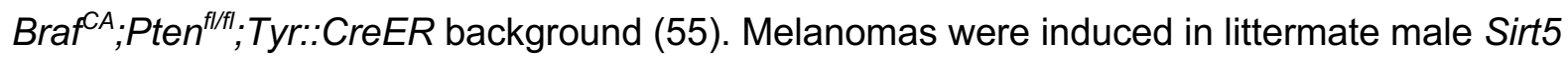
WT or Sirt5 KO mice as shown by topical application of 4HT at ages 4-9 weeks; tumors were weighed following euthanasia. Averages of 5 sets of male mice are plotted $(p<0.05$, paired twotailed t-test). Means \pm SD are shown. D. SIRT5 immunoblot of a representative tumor from a Sirt5 WT or KO male or female mouse (left panel). Representative tumor from a Sirt5 WT or KO male mouse, as indicated, after $4 \mathrm{HT}$ induction (right panel). Scale bar=1cm.

\section{Figure 5 Bioenergetics are maintained upon SIRT5 loss in melanoma cells.}

A2058 and A375 cells maintain glycolytic function (A.), glucose-dependent mitochondrial respiration (B.), and ATP production (C.) upon SIRT5 depletion compared to control cells. Mitochondrial respiration, glycolytic stress tests, and ATP production rates were measured at 72 hrs. post-transduction with shRNAs against SIRT5 using a Seahorse XFe96 Analyzer. All rates are normalized to total protein content per sample ( $n=6$ for $\mathbf{A}$. and $\mathbf{C}$., $n=5$ for B.). OCR, oxygen consumption rate; ECAR, extracellular acidification rate. Error bars represent standard deviation. D. Mitochondrial membrane potential is stable in A2058 cells after SIRT5 loss (C, control cells, $n=6$ ). Cells were incubated with JC-1, a dye which exhibits membrane potentialdependent accumulation in mitochondria, indicated by a fluorescence emission shift from green to red. Mitochondrial depolarization is indicated by a decrease in the red:green (aggregate:monomer) fluorescence intensity ratio. FCCP, a mitochondrial uncoupler, 
depolarizes mitochondrial membrane potential and is used as a positive control. Significance calculated using unpaired Student's t-test.

\section{Figure 6 Expression of MITF and MITF target genes is dependent upon SIRT5.}

Genes (A.) upregulated or (B.) downregulated upon SIRT5 KD. Only genes significantly $(p<0.05)$ altered in both KDs in each cell line, as indicated, were scored.

C. Expression levels of differentially expressed genes (DEGs; qadj<0.05) in response to SIRT5 KD were correlated with SIRT5 gene expression using Spearman's rank correlation coefficient in 443 sequenced human skin cutaneous melanoma (SKCM) samples, identifying DEGs with significant clinical correlation with SIRT5 expression $(q<0.01)$. Labeled genes represent oncogenes or extremely correlated genes most significantly altered by SIRT5 knockdown ( $q<0.0001$, log2FoldChange>2). D. Immunoblot demonstrating loss of MITF expression 96 hrs. post-transduction with shRNAs targeting SIRT5 (KD1 or KD2) compared to a non-targeting control $(C)$ in 5 cutaneous and one uveal melanoma cell lines, as indicated. E. Relative FPKMs in A2058 and SK-MEL-2 cells demonstrate a loss of MITF (bar graphs, upper panels) and several MITF target gene transcripts upon SIRT5 KD (heatmaps, lower panels). Scale bars adjacent to heat maps indicate linear fold change (control (C) set to 1). Significance calculated using unpaired Student's t-test. F. Expression of SIRT5, MITF and the MITF target, PPARGC1A are positively correlated in melanoma clinical samples $(p<0.0001$, data from TCGA, analyzed on cBioPortal; see Figure 1A).

\section{Figure 7 SIRT5 promotes histone acetylation in melanoma.}

A. Heatmap of z-scores calculated from metabolic reaction fluxes predicted by genome-scale modeling to be differentially active $(p<0.01)$ after SIRT5 KD. B. Total histone acetylation is reduced $96 \mathrm{hrs}$. post-transduction with shRNAs targeting SIRT5 (KD1 or KD2) compared to a non-targeting control $(\mathrm{C})$ in melanoma cell lines. Lanes were run on the same gel but are 
noncontiguous. C. Immunoblot demonstrating loss of H3K9ac and H4K16ac 96 hrs. posttransduction with shRNAs targeting SIRT5 (KD1 or KD2) compared to a non-targeting control (C) in A2058 cells. D. H3K9ac is reduced within the promoter regions of MITF and c-Myc in SIRT5-depleted A2058 cells via CUT\&RUN followed by qRT-PCR. Signal (Ct values) relative to input DNA were normalized to control (C) samples for each primer set. Graphed are averages of $\mathrm{n}=9$ replicates. Error bars represent SD. Significance calculated using unpaired Student's t-test. Acetylation (E.) and MITF expression (F.) are restored in A2058 cells lacking SIRT5 after 4 weeks of continual culture in puromycin. G. Total cellular acetyl-CoA levels are increased in A2058, A375 and SK-MEL-2 cells 96 hrs. after SIRT5 depletion. Acetyl-CoA abundance was quantified by liquid chromatography-high resolution mass spectrometry and normalized to cell number. Plotted are average $(n=5)$ acetyl-CoA levels as pmol acetyl-CoA $/ 10^{5}$ cells. Error bars represent SD. Significance calculated using unpaired Student's t-test.

\section{Figure 8 SIRT5 promotes histone methylation and reduced cellular ROS levels in}

\section{melanoma.}

A. LC-MS/MS-based metabolite profiling followed by MetaboAnalyst pathway analysis demonstrate alterations in glycine and serine and methionine biosynthesis pathways in melanoma cells upon SIRT5 depletion. B. Perturbations in 1C metabolite levels in response to SIRT5 loss in the cell lines shown. Each column represents the mean of 3 independently prepared biological replicates. Metabolite levels in SIRT5 depleted (KD1 and KD2, as indicated) samples are normalized to control. SAM, S-adenosyl-methionine; SAH, Sadenosylhomocysteine; GSH, reduced glutathione; GSSG, glutathione disulfide. C. H3K4me3 and H3K9me3 immunoblot in melanoma cells $96 \mathrm{hrs}$. post-transduction with shRNAs targeting SIRT5 (KD1 or KD2) compared to a non-targeting control (C). D. H3K4me3 and H3K9me3 levels are restored in A2058 cells lacking SIRT5 after 4 weeks of continual culture in puromycin. E. Flow cytometric analysis of DCFDA-stained A2058 cells 96 hrs. post-transduction with 
shRNAs targeting SIRT5 (KD1 or KD2) reveals increased ROS compared to a non-targeting control $(C), p<0.005$. Left panel, average mean fluorescence intensity of DCFDA positive populations in $n=3$ samples. Error bars represent SD. Significance calculated using unpaired Student's t-test. Right panel, representative flow cytometric of A2058 cells stained with DCFDA. F. SIRT5 interacts with MTHFD1L in A2058 cells. Increasing amounts of anti-SIRT5 antibody increases SIRT5-MTHFD1L coprecipitation compared to normal rabbit IgG control. Basal expression of SIRT5 and MTHFD1L in whole-cell extract (1\% of initial amount used for immunoprecipitation) is shown for comparison. G. Proposed model of promotion of MITF and cMYC expression via SIRT5-dependent chromatin modifications in human melanoma. Me, methylation; Ac, acetylation. 


\section{Figure 1}

A.

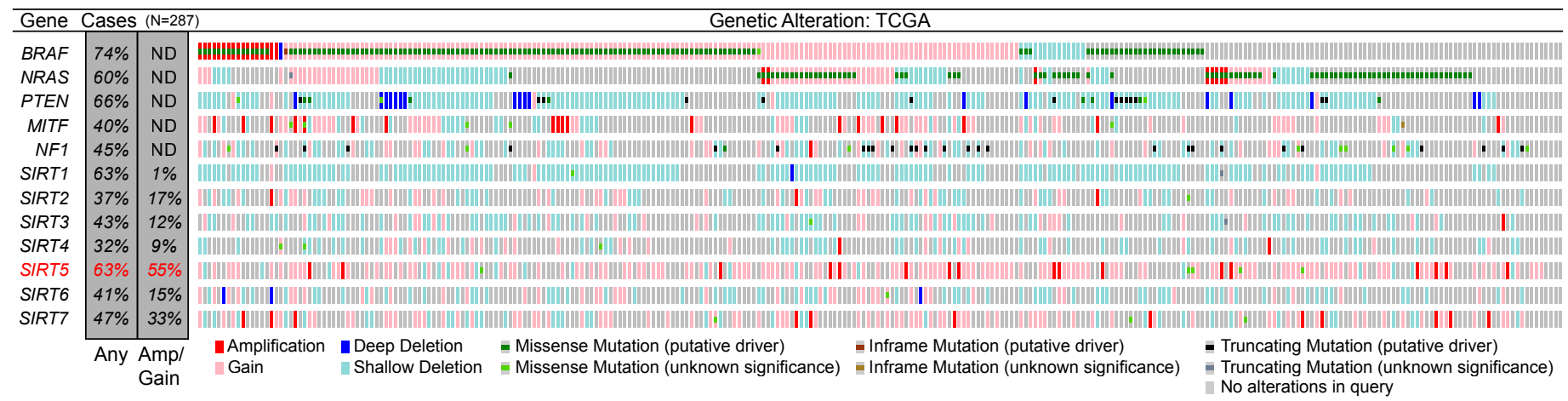

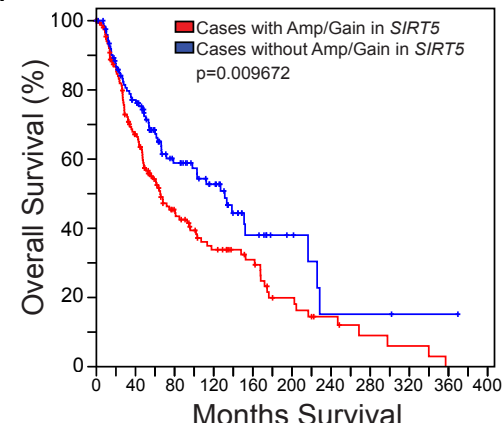

C.

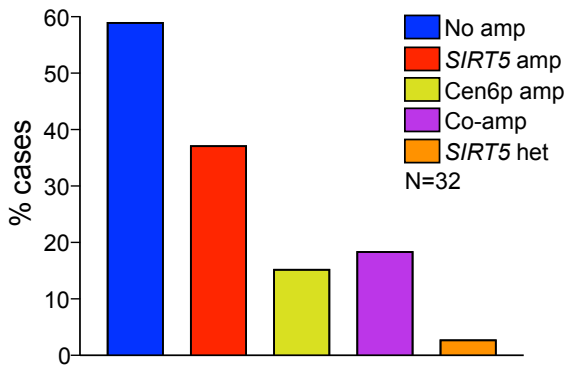

D.

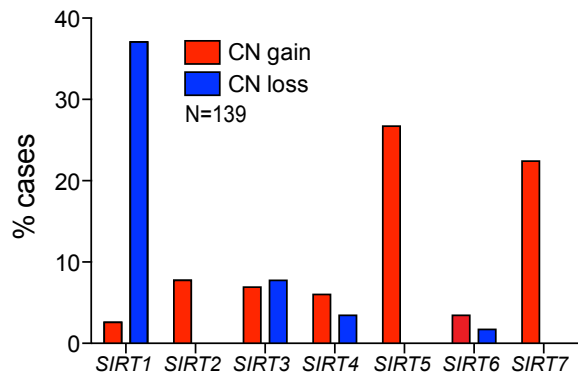

E.

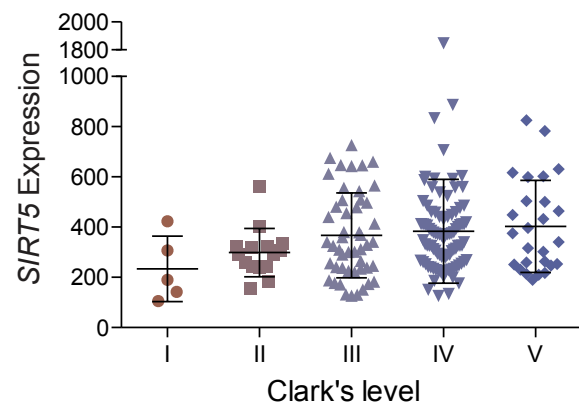

F.

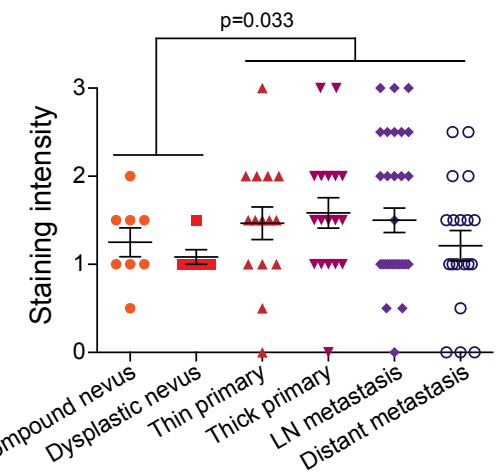

Figure 1 Increased SIRT5 copy number in human melanoma.

A. Gain of extra SIRT5 copies in melanoma. BRAF, NRAS, PTEN, MITF, NF1 and other sirtuins are shown for comparison ( $\mathrm{n}=287$; data from TCGA, Provisional, analyzed on cBioPortal). $\mathrm{ND}$, not determined. Percentage of samples with any genomic alteration (Any) or amplification or gain (Amp/Gain) is indicated. Graphed are any alterations queried for the indicated gene. Copy number gain indicates a low-level gain of a single additional copy, and amplification refers to high-level amplification (multiple extra copies). Results from the query (GENE: MUT AMP HOMDEL GAIN HETLOSS) in cBioPortal were analyzed and plotted. B. Kaplan-Meier analysis of overall survival in melanoma patients with or without copy number gain or amplification of SIRT5. Overall survival was analyzed using the query: "SIRT5: AMP GAIN." C. SIRT5 (6p23) and centromere 6p (Cen6p) amplification (amp) or co-amplification (Co-amp) in melanoma, as assayed by FISH staining $(n=32)$. D. Sirtuin gene copy number $(C N)$ in human melanoma samples, as assayed by high density SNP array ( $n=139)$. E. SIRT5 mRNA expression levels in melanoma correlate with Clark's level ( $p=0.0044$, linear regression; $p=0.037$, ANOVA). F. SIRT5 protein levels are increased in melanoma relative to benign melanocytic lesions ( $p=0.0333$, Chi-squared; $n=14$ nevi, $n=87$ melanoma). See also Figure S1 and Table S1. 
bioRxiv preprint doi: https://doi.org/10.1101/2020.09.07.286526; this version posted February 24, 2021. The copyright holder for this preprint

Figure 2 (which was not certified by peer review) is the author/funder. All rights reserved. No reuse allowed without permission.

A.
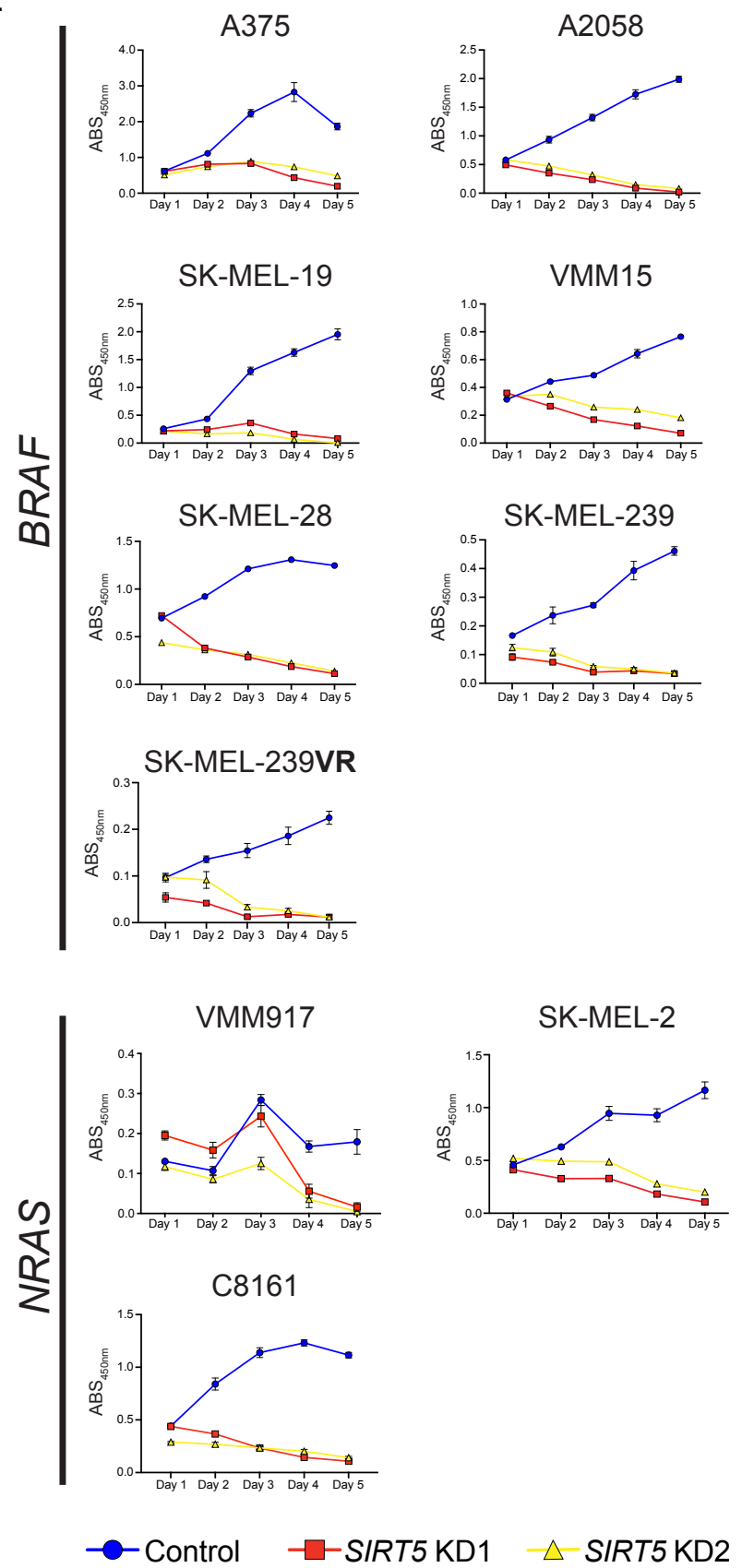

VMM15

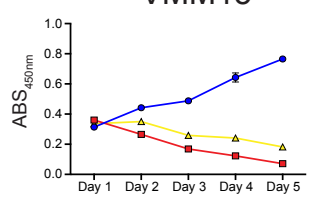

SK-MEL-239

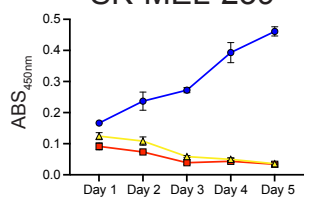

B.

A2058

C

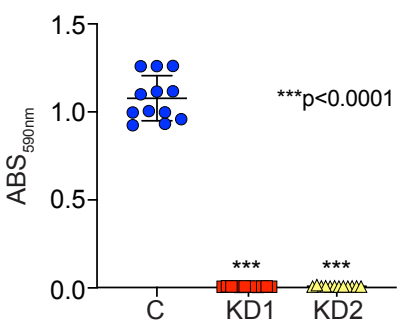

A2058
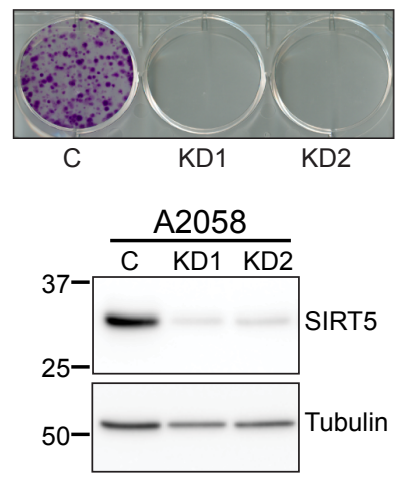

C.

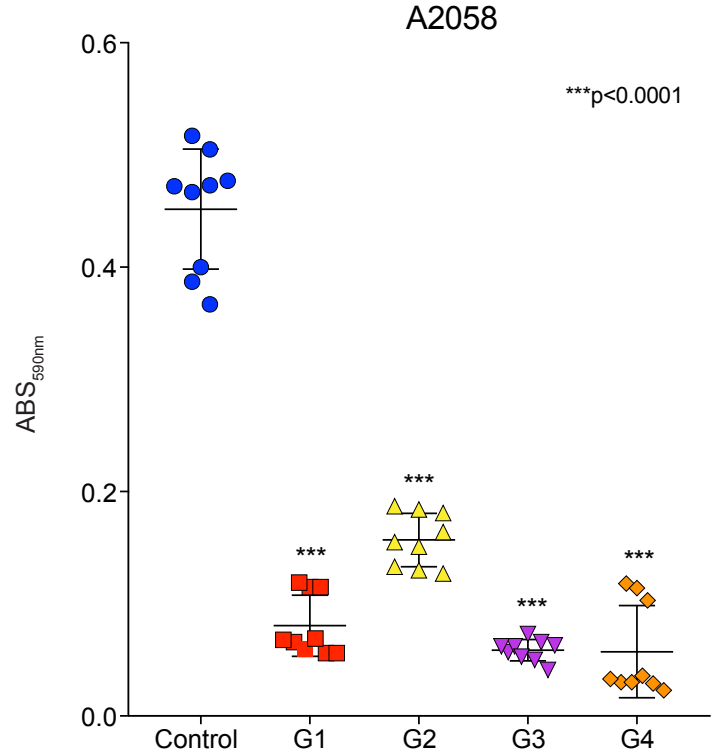

SK-MEL-2
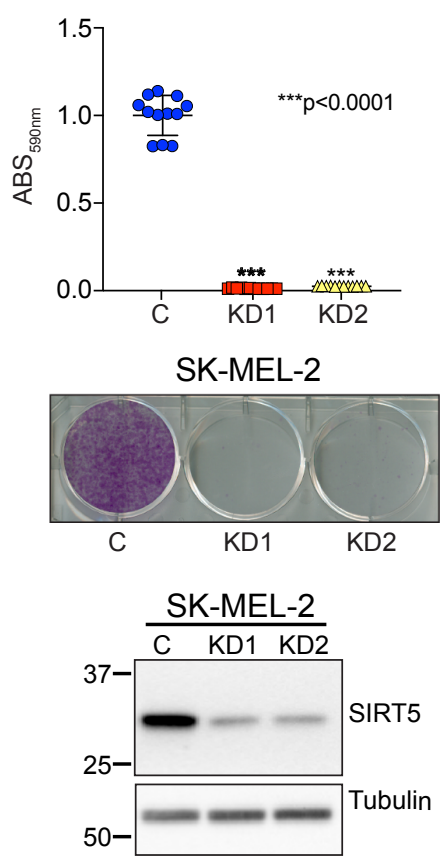

A2058

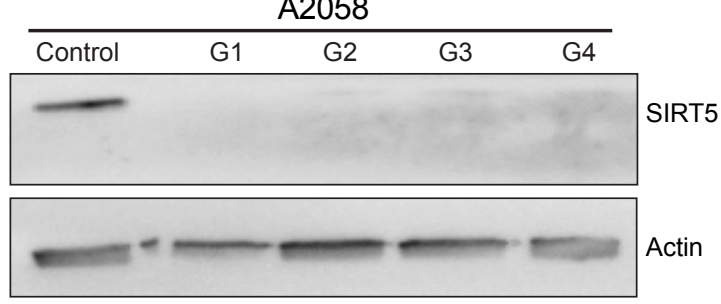

Figure 2 SIRT5 is required for melanoma cell growth and survival.

A. BRAF or NRAS mutant melanoma cell lines indicated were infected with a non-targeting shRNA (control) or one of two SIRT5 shRNAs (KD1 or KD2). Equivalent cell numbers were then plated $48 \mathrm{hrs}$. post-transduction into 96 -well plates in the presence of puromycin. Cell mass was determined at the indicated timepoints via WST-1 assay, with absorbance measured at $450 \mathrm{~nm}$. Average results ( $\mathrm{n}=6 /$ timepoint) are graphed. Error bars represent standard deviation. Representative of $5 / 5$ SIRT5 shRNAs tested (see also Figure 3B). B. SIRT5 KD results in significantly ( $p<0.0001$, unpaired Student's t-test) impaired colony formation by A2058 and SK-MEL-2 cells 12 days post-transduction. Cell mass was assayed using crystal violet staining, with absorbance measured at $590 \mathrm{~nm}$. Average of $\mathrm{n}=12$ technical replicates results are plotted. Error bars represent SD. Representative crystal violet-stained wells are shown. Lower panel, representative immunoblot analysis demonstrating SIRT5 KD. C. Top panel, viability of A2058 cells transfected with the indicated CRISPR guide RNA (Control or G1-G4). Cell mass was assayed using crystal violet staining, with absorbance measured at $590 \mathrm{~nm}$. Average of $n=9$ technical replicates results are plotted. Error bars represent standard deviation. Significance calculated using unpaired Student's t-test. Bottom panel, representative immunoblot analysis confirming CRISPR-mediated SIRT5 loss (Control: empty vector). 
bioRxiv preprint doi: https://doi.org/10.1101/2020.09.07.286526; this version posted February 24, 2021. The copyright holder for this preprint Figure 3 (which was not certified by peer review) is the author/funder. All rights reserved. No reuse allowed without permission.

A.
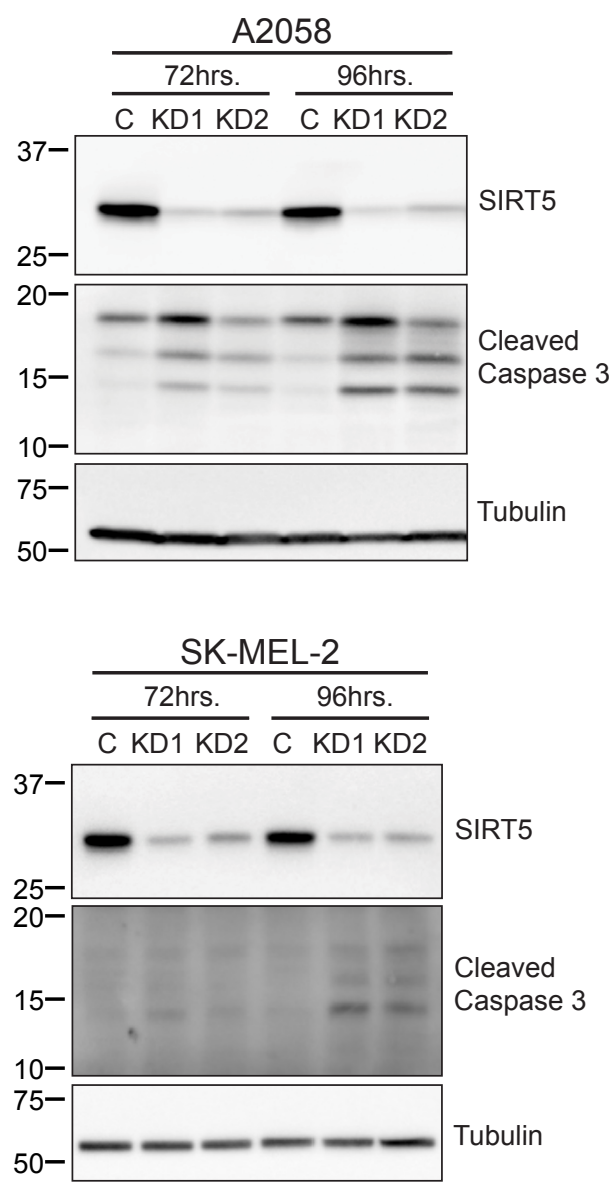

C.
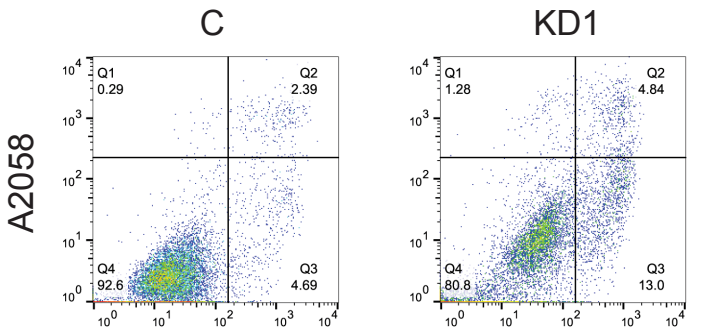

B.
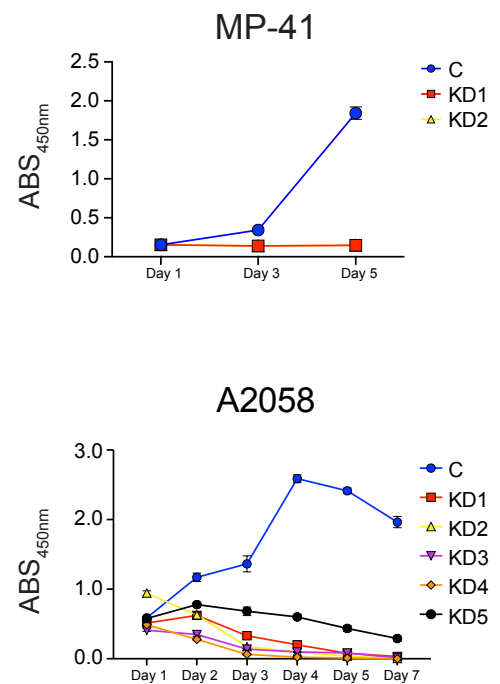

YUMM 5.2

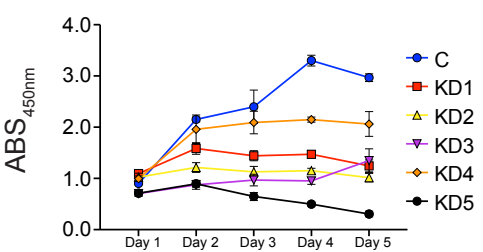

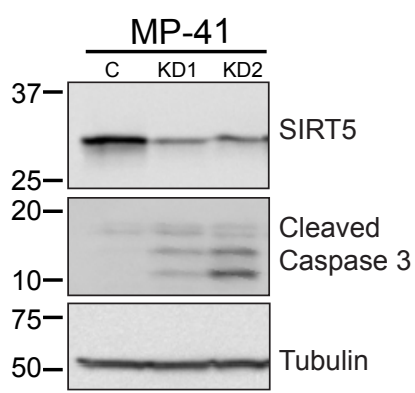

A2058

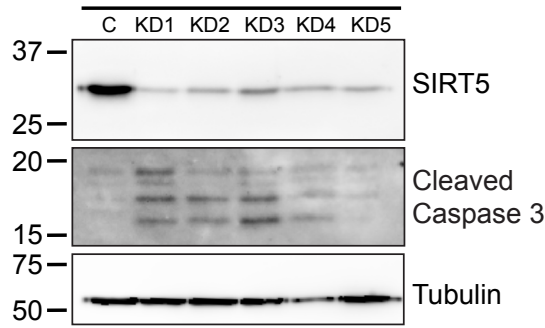

YUMM 5.2

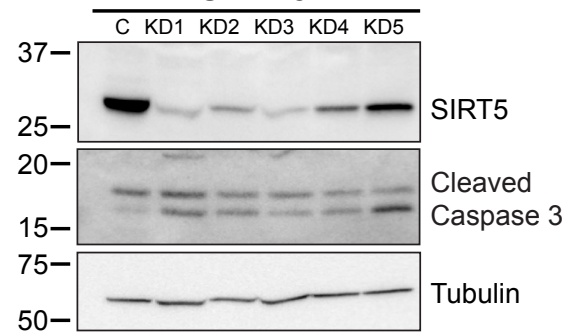

D.

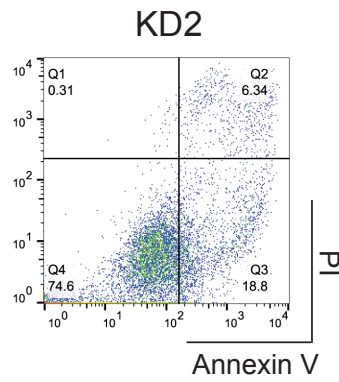

A2058

Figure 3 SIRT5 depletion rapidly induces apoptosis in melanoma cells.

A. Immunoblot analysis demonstrating induction of caspase 3 cleavage 72 and 96 hrs. post-transduction with shRNAs targeting SIRT5 (KD1 or KD2) in A2058 and SK-MEL-2 cell lines. B. Viability of MP-41, A2058 or YUMM5.2 cells infected with control (C) or one of five SIRT5 shRNAs (KD1-KD5) against human SIRT5 (top and middle panels) or mouse Sirt5 (bottom panel). Average results ( $n=6 /$ timepoint) are graphed. Error bars represent standard deviation. Right panels: immunoblot analysis demonstrating loss of SIRT5 and induction of caspase 3 cleavage following SIRT5 KD. C. Flow cytometric analysis of A2058 cells stained with Annexin V and propidium iodide (PI), as indicated, showing an increased fraction of Annexin V-positive cells $96 \mathrm{hrs}$. after SIRT5 KD. D. Average of $n=3$ technical replicates is plotted. Error bars represent (SD). Significance calculated using unpaired Student's t-test. Increased Annexin $\mathrm{V}^{+}$staining is observed in both the PI-positive and PI-negative populations. 
bioRxiv preprint doi: https://doi.org/10.1101/2020.09.07.286526; this version posted February 24, 2021. The copyright holder for this preprint Figure 4 (which was not certified by peer review) is the author/funder. All rights reserved. No reuse allowed without permission.

A.

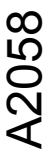

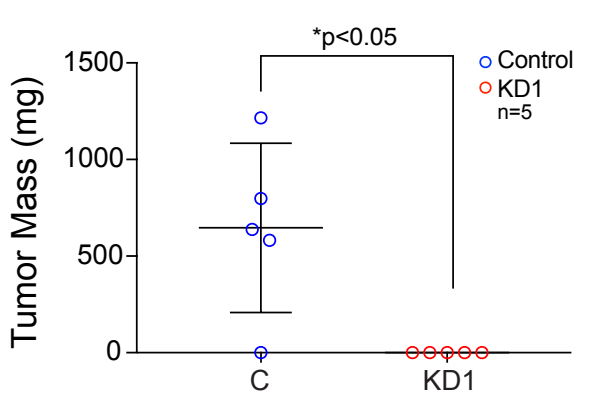

B.
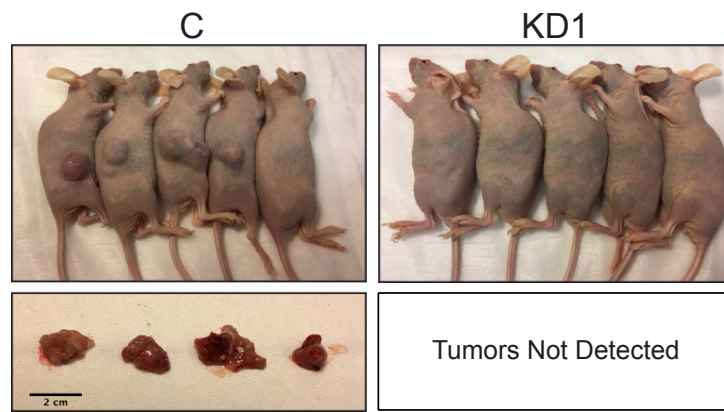

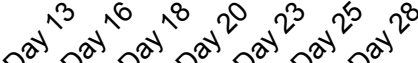

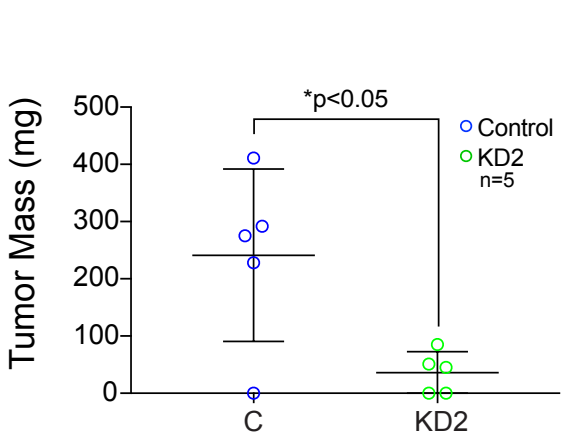

C

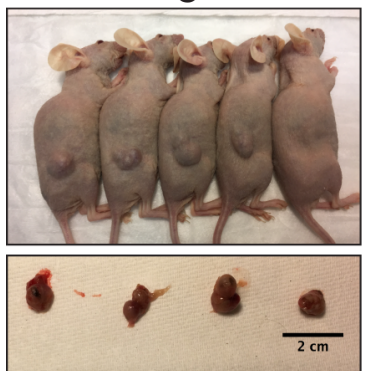

KD2

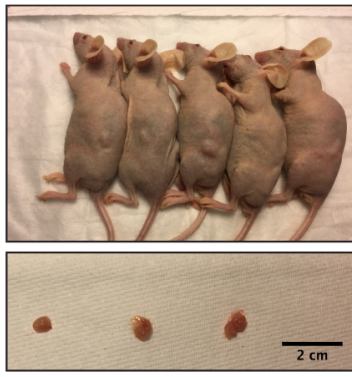

C.

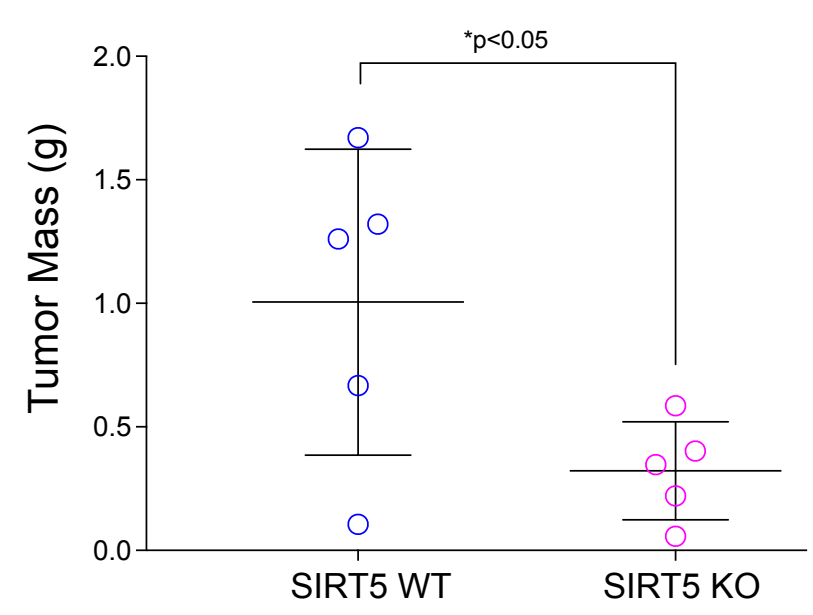

D.

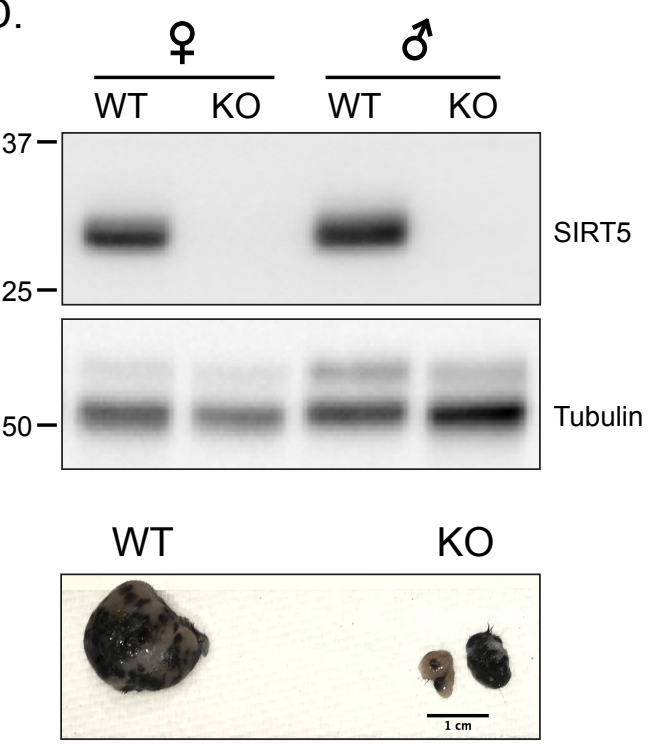

Figure 4 SIRT5 loss-of-function inhibits melanoma tumor growth in vivo.

A. SIRT5 depletion in A2058 cells results in attenuated xenograft tumor growth. Quantification of tumor size was initiated on day 13 after initial injection of cells (left panel). Tumor size was recorded with Vernier calipers on the days indicated. Each point represents the measurements on $n=5$ mice for each condition (C, KD1, or KD2). Pairwise representation of endpoint tumor size in each mouse within each group is plotted (right panel). Average tumor mass measurements at day 28 are plotted $(p<0.05$, paired two-tailed t-test for each group). Error bars represent SD. B. Mice were sacrificed, and tumors were dissected at 28 days after initial injection. Scale bar below tumors $=2 \mathrm{~cm}$. C. SIRT5 deficiency attenuates tumor formation in an autochthonous melanoma model. Sirt5 deficient-mice were bred into the BrafCA;Pten flffl:Tyr::CreER background (55). Melanomas were induced in littermate male Sirt5 WT or Sirt5 KO mice as shown by topical application of $4 \mathrm{HT}$ at ages 4-9 weeks; tumors were weighed following euthanasia. Averages of 5 sets of male mice are plotted $(p<0.05$, paired two-tailed t-test). Means \pm SD are shown. D. SIRT5 immunoblot of a representative tumor from a Sirt5 WT or KO male or female mouse (left panel). Representative tumor from a Sirt5 WT or KO male mouse, as indicated, after $4 \mathrm{HT}$ induction (right panel). Scale bar= $1 \mathrm{~cm}$. 
C bignxiv preprint doi: https://doi.org/10.1101/2020.09.07.286526; this version posted February 24, 2021. The copyright holder for this preprint

F Igure 5 (which was not certified by peer review) is the author/funder. All rights reserved. No reuse allowed without permission.

A.

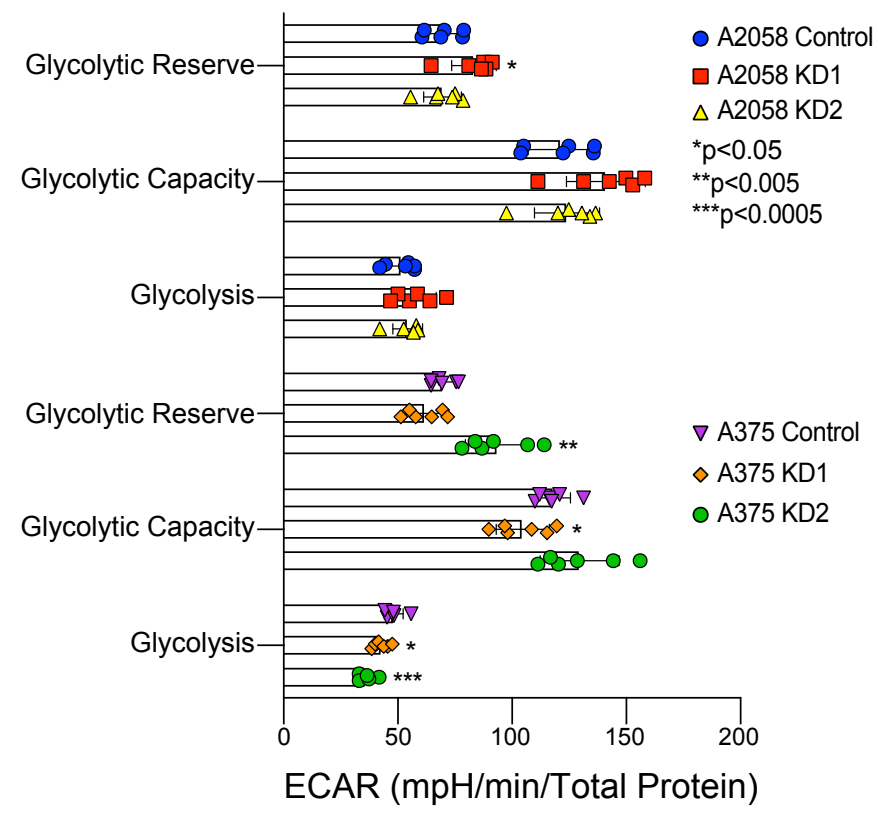

C.

ATP Production Rate

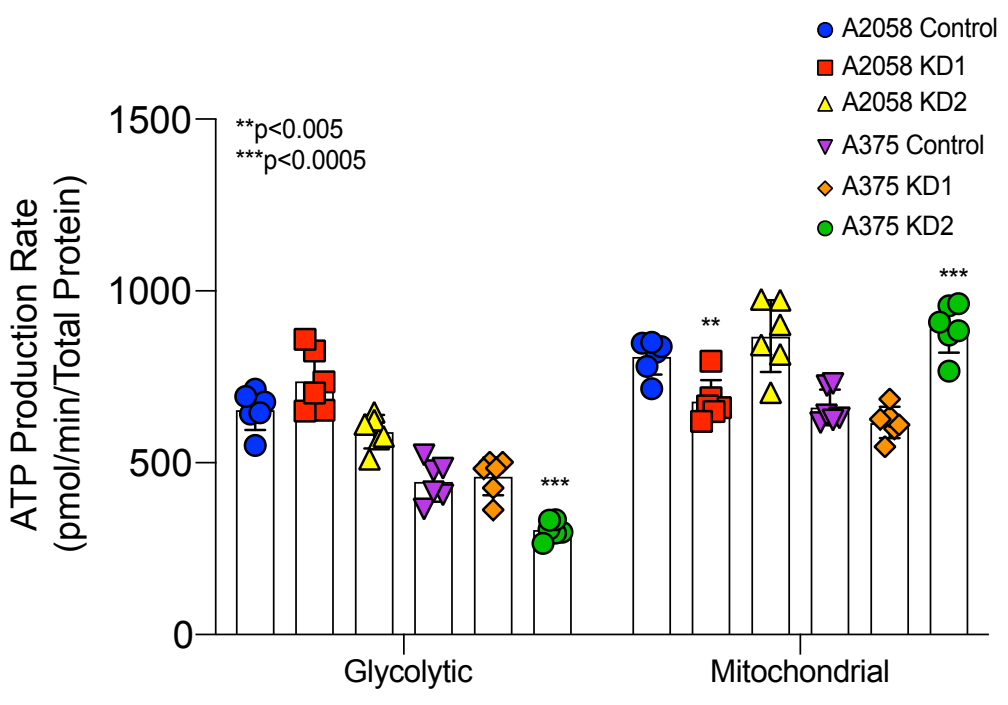

B.

Glucose-Dependent Mitochondrial Respiration

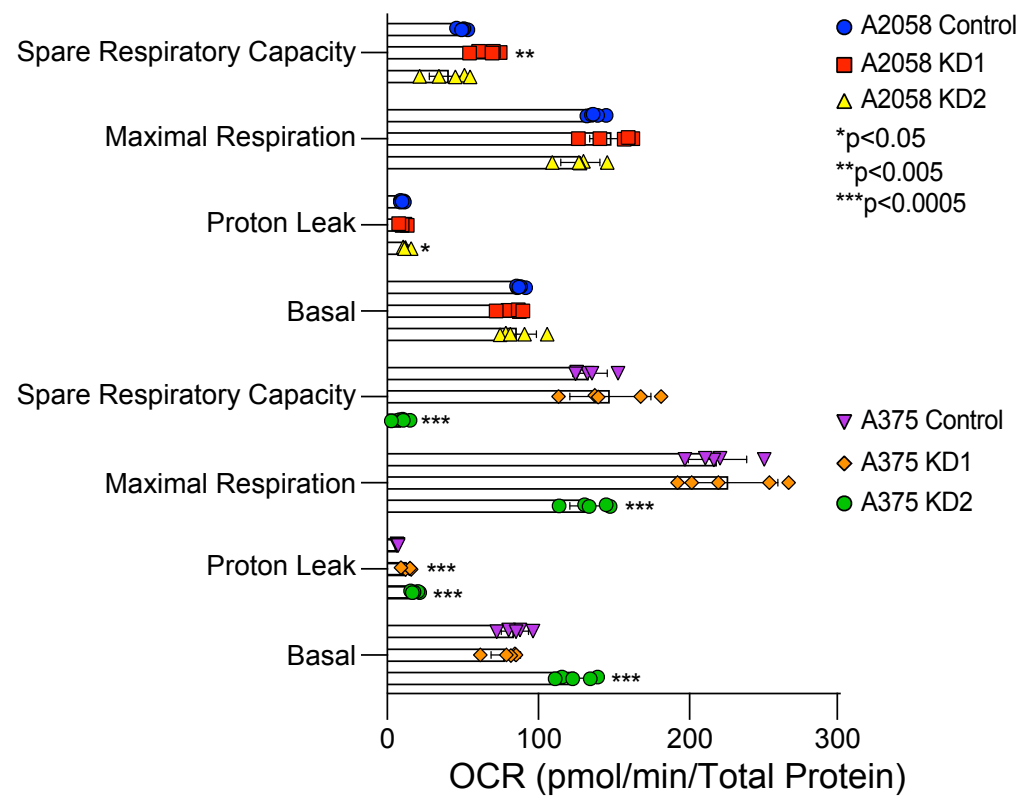

D.

\section{Mitochondrial Membrane Potential}

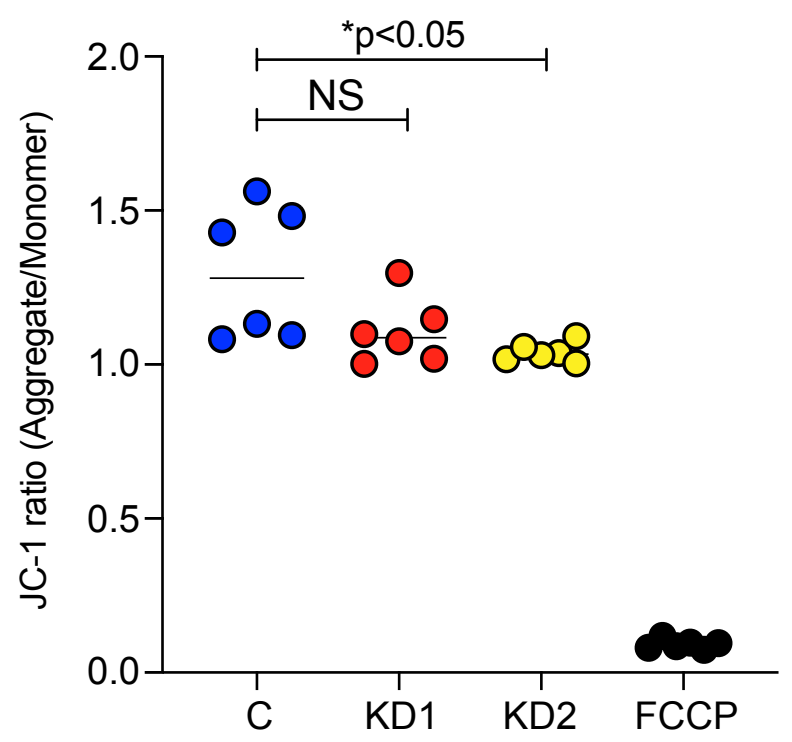

Figure 5 Bioenergetics are maintained upon SIRT5 loss in melanoma cells.

A2058 and A375 cells maintain glycolytic function (A.), glucose-dependent mitochondrial respiration (B.), and ATP production (C.) upon SIRT5 depletion compared to control cells. Mitochondrial respiration, glycolytic stress tests, and ATP production rates were measured at 72 hrs. post-transduction with shRNAs against SIRT5 using a Seahorse XFe96 Analyzer. All rates are normalized to total protein content per sample ( $n=6$ for $\mathbf{A}$. and $\mathbf{C}$., $n=5$ for B.). OCR, oxygen consumption rate; ECAR, extracellular acidification rate. Error bars represent standard deviation. D. Mitochondrial membrane potential is stable in A2058 cells after SIRT5 loss (C, control cells, $\mathrm{n}=6$ ). Cells were incubated with JC-1, a dye which exhibits membrane potential-dependent accumulation in mitochondria, indicated by a fluorescence emission shift from green to red. Mitochondrial depolarization is indicated by a decrease in the red:green (aggregate:monomer) fluorescence intensity ratio. FCCP, a mitochondrial uncoupler, depolarizes mitochondrial membrane potential and is used as a positive control. Significance calculated using unpaired Student's t-test. 
bioRxiv preprint doi: https://doi.org/10.1101/2020.09.07.286526; this version posted February 24, 2021. The copyright holder for this preprint Figurencon was not certified by peer review) is the author/funder. All rights resetyed. No reuse allowed withoutpermission.

A.

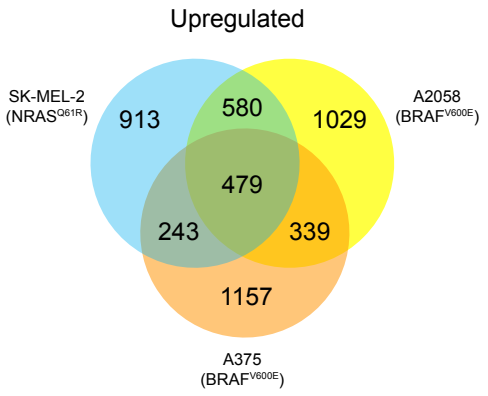

B.

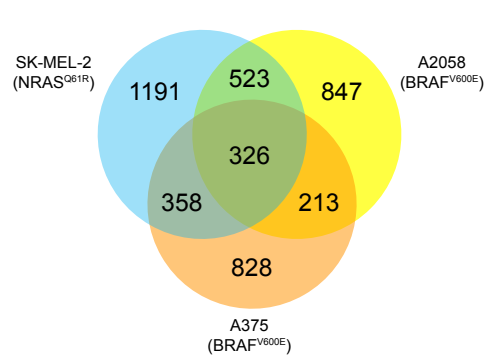

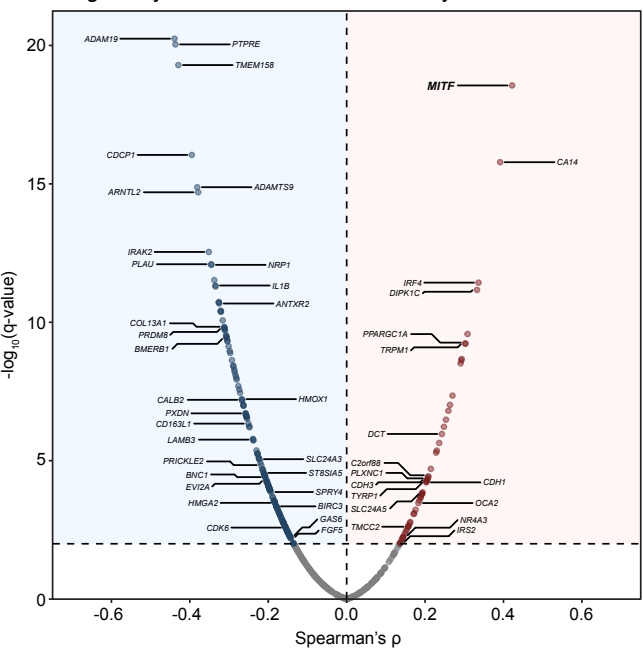
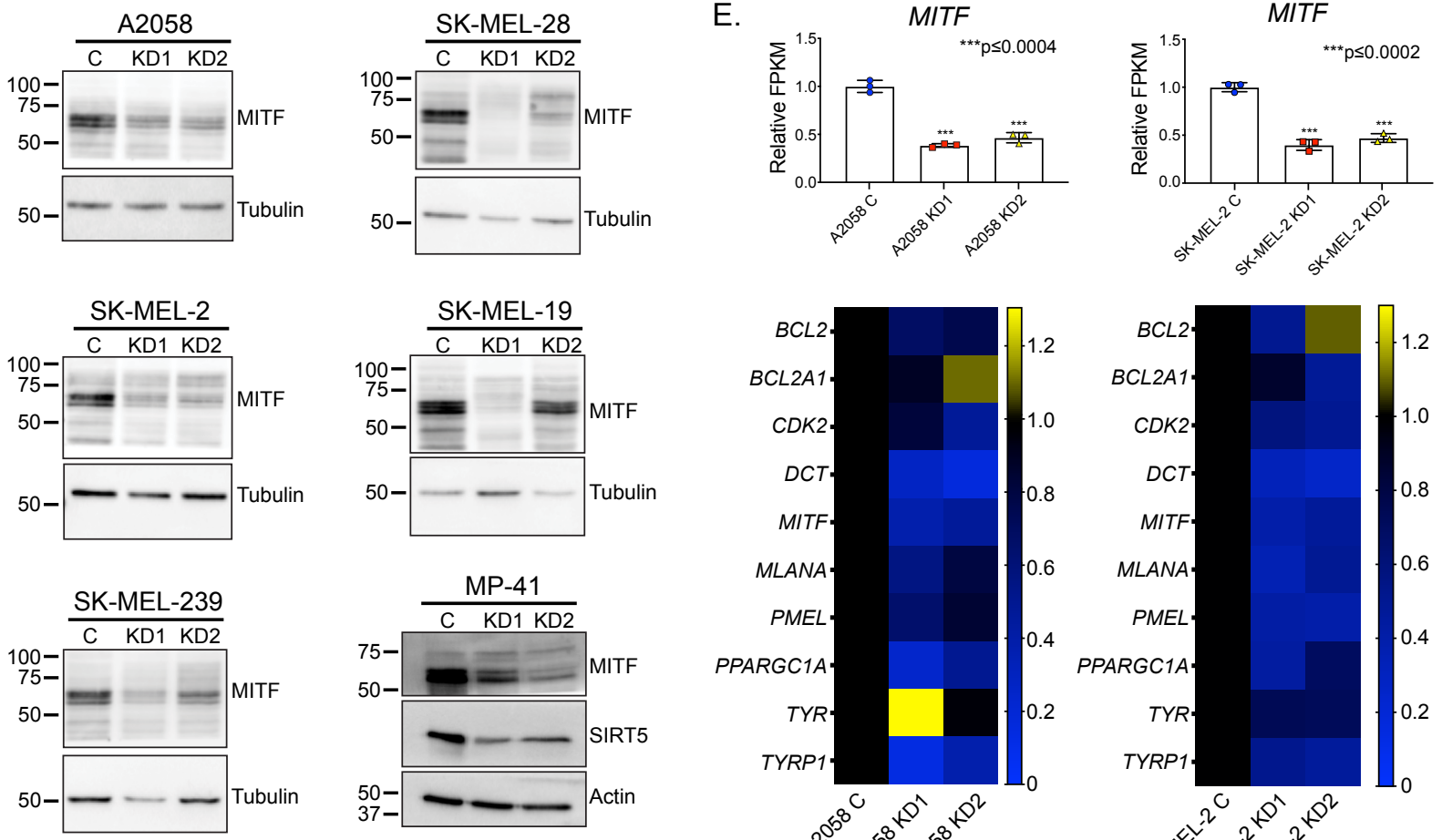

E.
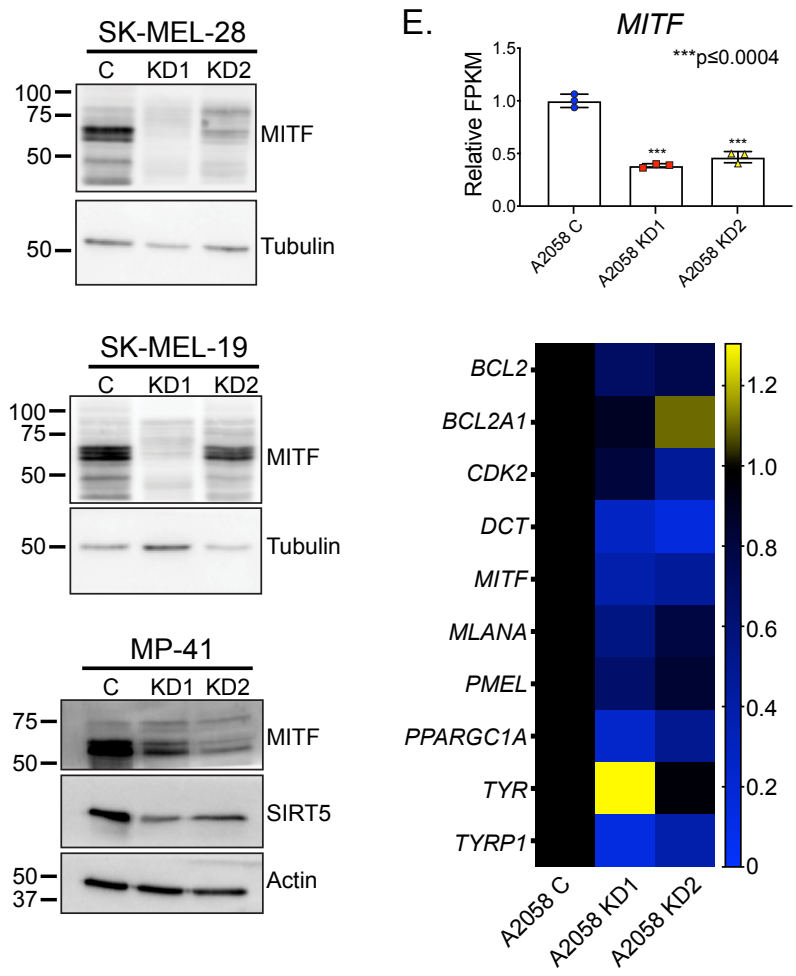

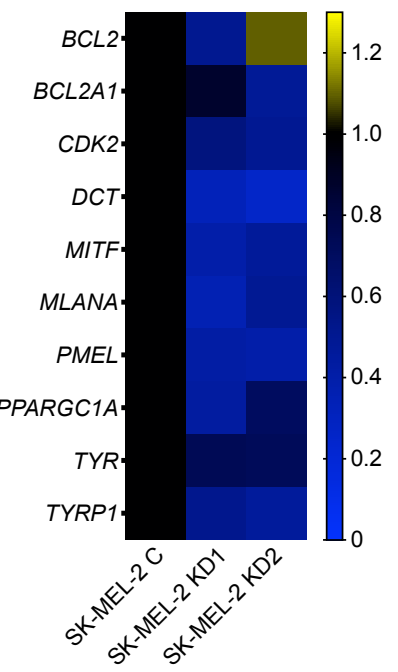

F.
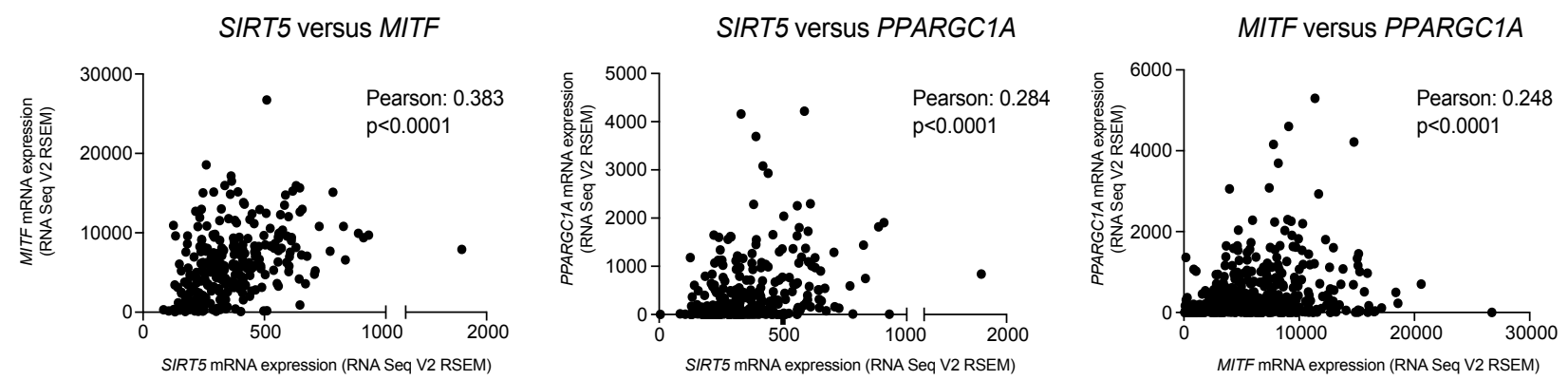

Figure 6 Expression of MITF and MITF target genes is dependent upon SIRT5.

Genes (A.) upregulated or (B.) downregulated upon SIRT5 KD. Only genes significantly $(p<0.05)$ altered in both KDs in each cell line, as indicated, were scored. C. Expression levels of differentially expressed genes (DEGs; qadj<0.05) in response to SIRT5 KD were correlated with SIRT5 gene expression using Spearman's rank correlation coefficient in 443 sequenced human skin cutaneous melanoma (SKCM) samples, identifying DEGs with significant clinical correlation with SIRT5 expression $(\mathrm{q}<0.01$ ). Labeled genes represent oncogenes or extremely correlated genes most significantly altered by SIRT5 knockdown ( $q<0.0001$, log2FoldChange>2). D. Immunoblot demonstrating loss of MITF expression 96 hrs. post-transduction with shRNAs targeting SIRT5 (KD1 or KD2) compared to a non-targeting control (C) in 5 cutaneous and one uveal melanoma cell lines, as indicated. E. Relative FPKMs in A2058 and SK-MEL-2 cells demonstrate a loss of MITF (bar graphs, upper panels) and several MITF target gene transcripts upon SIRT5 KD (heatmaps, lower panels). Scale bars adjacent to heat maps indicate linear fold change (control (C) set to 1). Significance calculated using unpaired Student's t-test. F. Expression of SIRT5, MITF and the MITF target, PPARGC1A are positively correlated in melanoma clinical samples ( $p<0.0001$, data from TCGA, analyzed on cBioPortal; see Figure $1 \mathrm{~A})$. 


\section{Figure 7}

A.<smiles>CCCC(CO)C[TeH]</smiles>

Pyruvate kinase

Pyruvate dehydrogenase

ATP-Citrate lyase

Phosphofructokinase

Cytidylate kinase (dCMP,UTP)

24-Dehydrocholesterol reductase [Precursor]

7-Dehydrocholesterol reductase. 1

Lathosterol oxidase.1

Retinol dehydrogenase (9-cis, NADH)

Retinol dehydrogenase (11-cis, NADH)

Formate dehydrogenase

Cytidylate kinase (CMP)

dCMP deaminase

Methylenetetrahydrofolate dehydrogenase, mitochondrial $3-\beta$-Hydroxysteroid- $\delta(8), \delta(7)$-isomerase Phosphatidate cytidylyltransferase

Retinol dehydrogenase (11-cis, NADPH)

7-Dehydrocholesterol reductase

Lathosterol oxidase

Retinol dehydrogenase (9-cis, NADPH)
B.

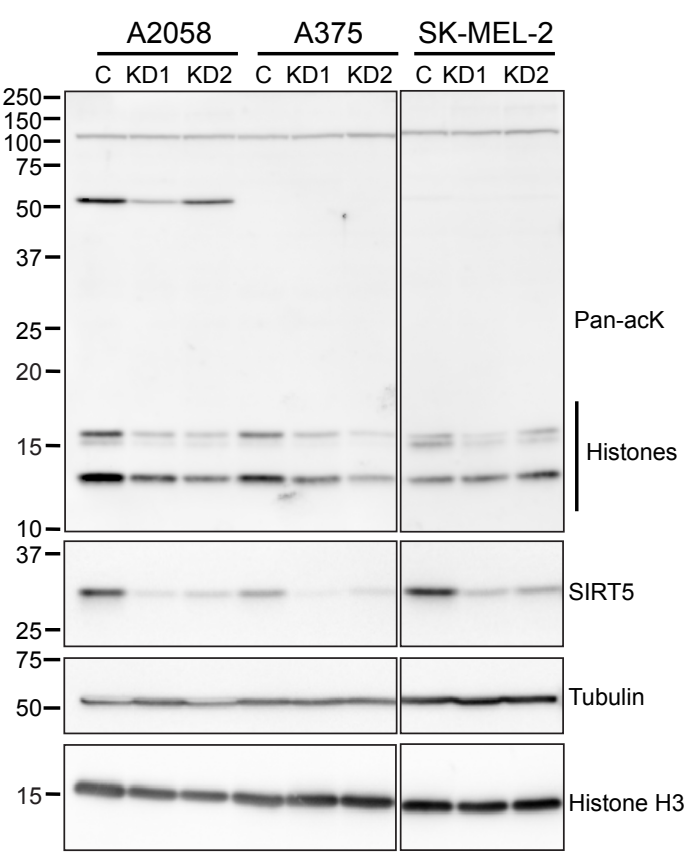

C.

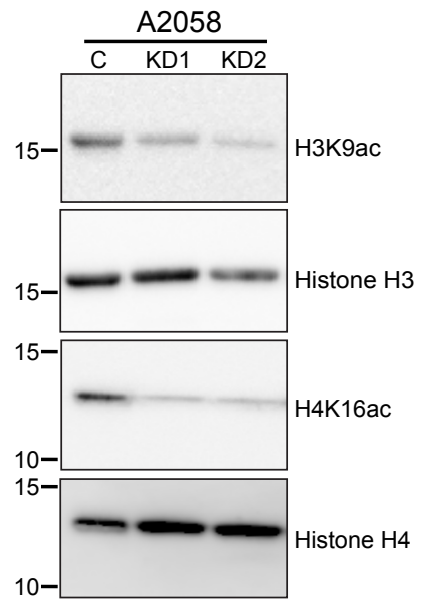

D.

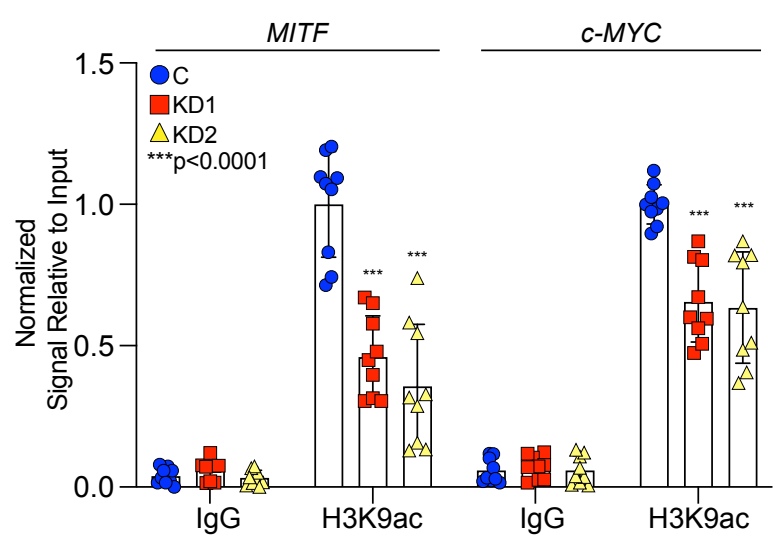

E.

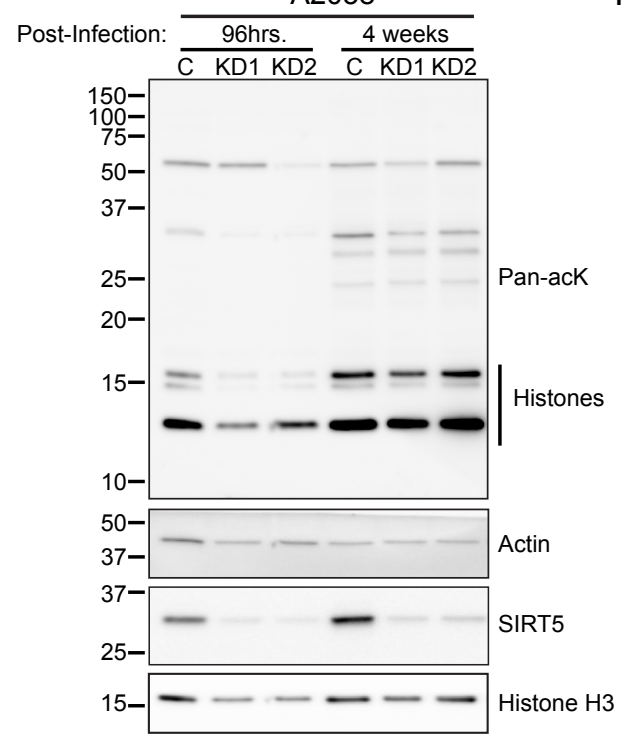

F. Post-Infection:

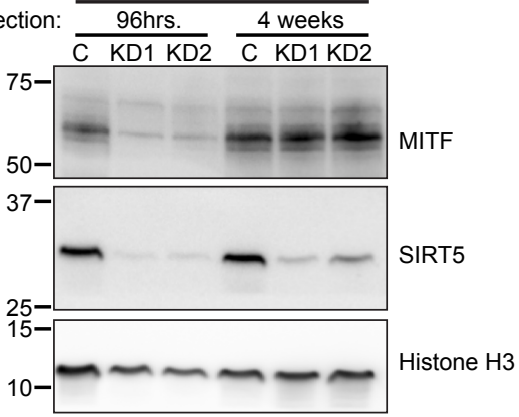

G.

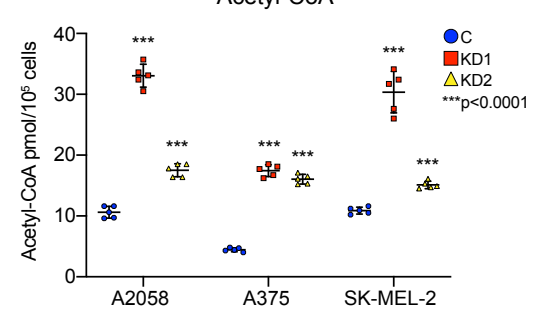

Figure 7 SIRT5 promotes histone acetylation in melanoma.

A. Heatmap of z-scores calculated from metabolic reaction fluxes predicted by genome-scale modeling to be differentially active $(p<0.01)$ after SIRT5 KD. B. Total histone acetylation is reduced 96 hrs. post-transduction with shRNAs targeting SIRT5 (KD1 or KD2) compared to a non-targeting control (C) in melanoma cell lines. Lanes were run on the same gel but are noncontiguous. C. Immunoblot demonstrating loss of H3K9ac and H4K16ac 96 hrs. post-transduction with shRNAs targeting SIRT5 (KD1 or KD2) compared to a non-targeting control (C) in A2058 cells. D. H3K9ac is reduced within the promoter regions of MITF and c-Myc in SIRT5-depleted A2058 cells via CUT\&RUN followed by qRT-PCR. Signal (Ct values) relative to input DNA were normalized to control (C) samples for each primer set. Graphed are averages of $n=9$ replicates. Error bars represen SD. Significance calculated using unpaired Student's t-test. Acetylation (E.) and MITF expression (F.) are restored in A2058 cells lacking SIRT5 after 4 weeks of continual culture in puromycin. G. Total cellular acetyl-Co
levels are increased in A2058, A375 and SK-MEL-2 cells 96 hrs. after SIRT5 depletion. Acetyl-CoA abundance was quantified by liquid chromatography-high resolution mass spectrometry and normalized to cell number Plotted are average $(n=5)$ acetyl-CoA levels as pmol acetyl-CoA/105 cells. Error bars represent SD. Significance calculated using unpaired Student's t-test. 


\section{Figure 8}

A.

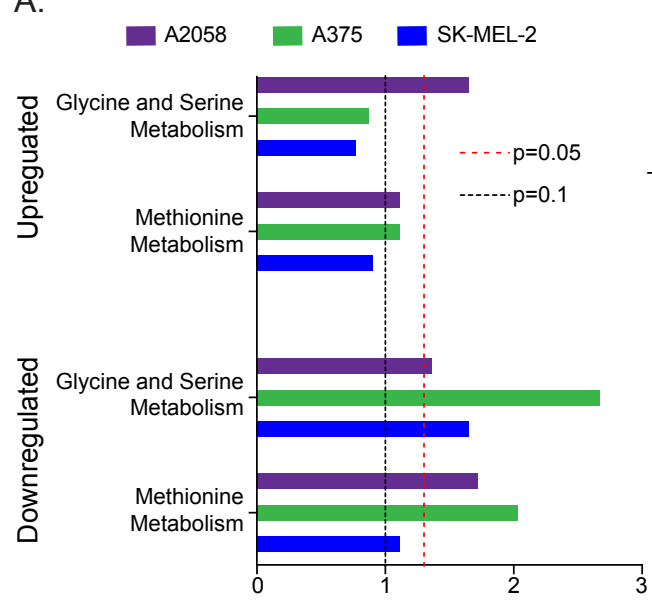

-log(p-value)
D.

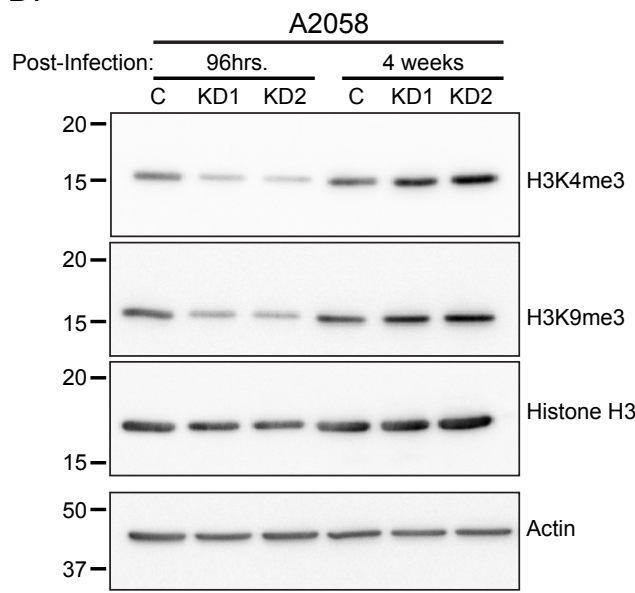

B. $\frac{\text { A2058 }}{\text { KD1 KD2 }} \frac{\text { A375 }}{\text { KD1 KD2 }}$ SK-MEL-2

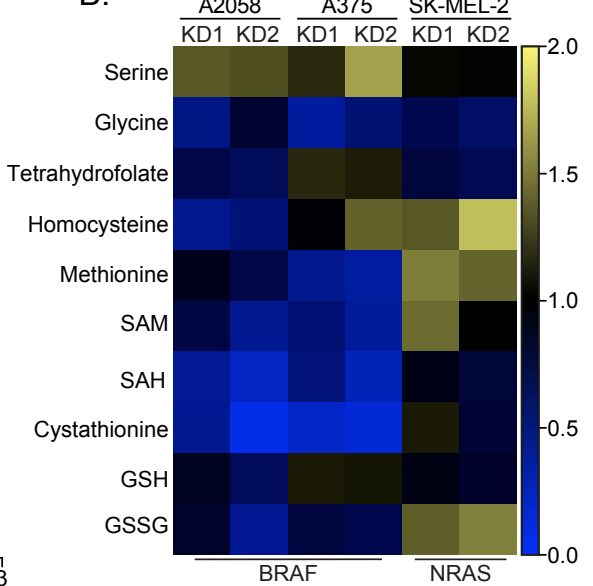

$\pi^{2.0}$ $-1.5$

$-1.0$

E.
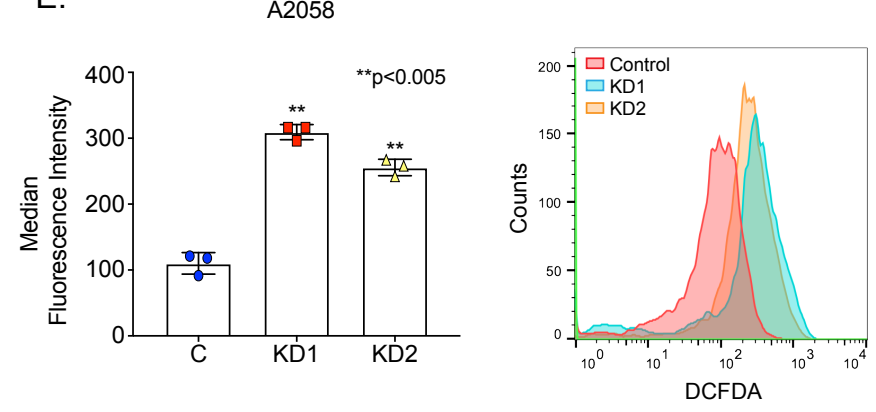

F.

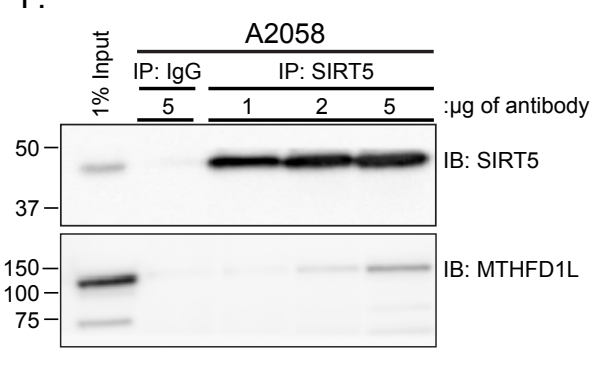

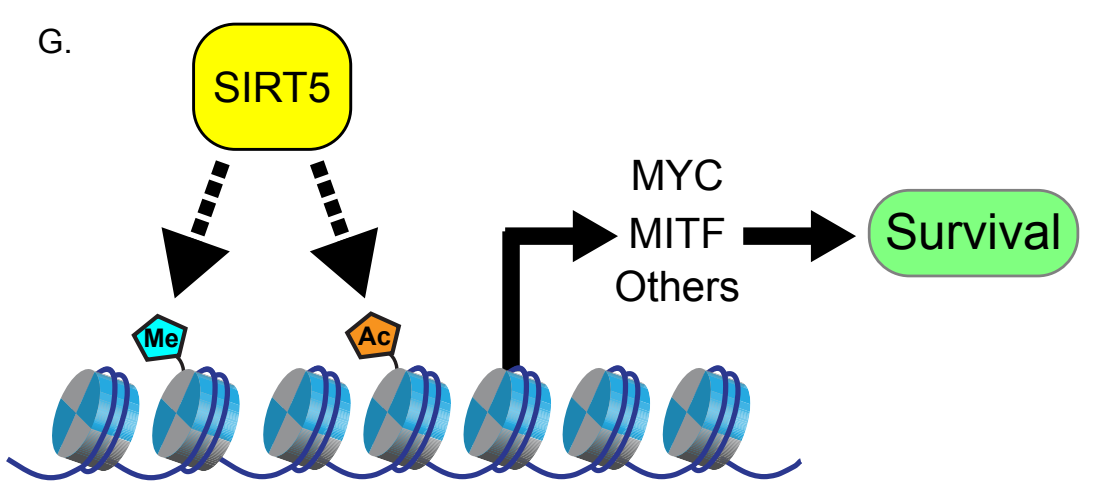

Figure 8 SIRT 5 promotes histone methylation and reduced cellular ROS levels in melanoma.

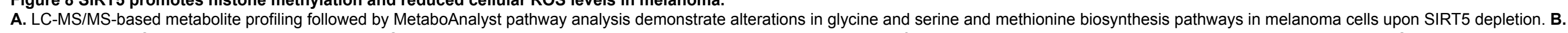

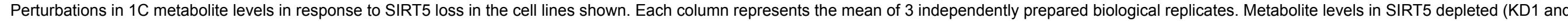

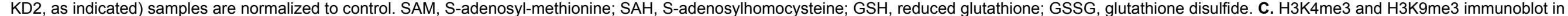

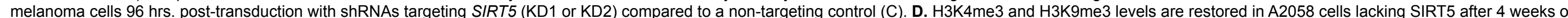

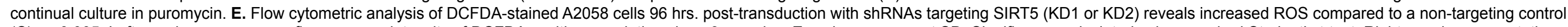

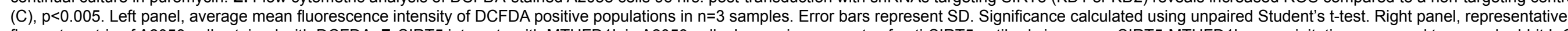

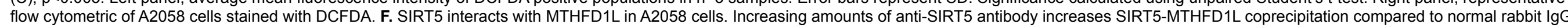

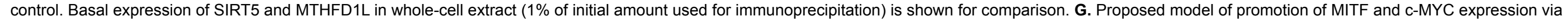
SIRT5-dependent chromatin modifications in human melanoma. Me, methylation; Ac, acetylation. 